\title{
THE INVERSE PROBLEM FOR POWER DISTRIBUTIONS IN COMMITTEES
}

\author{
SASCHA KURZ
}

\begin{abstract}
Several power indices have been introduced in the literature in order to measure the influence of individual committee members on the aggregated decision. Here we ask the inverse question and aim to design voting rules for a committee such that a given desired power distribution is met as closely as possible. We present an exact algorithm for a large class of different power indices based on integer linear programming. With respect to negative approximation results we generalize the approach of Alon and Edelman who studied power distributions for the Banzhaf index, where most of the power is concentrated on few coordinates. It turned out that each Banzhaf vector of an $n$-member committee that is near to such a desired power distribution, has to be also near to the Banzhaf vector of a $k$-member committee. We show that such Alon-Edelman type results are possible for other power indices like e.g. the Public Good index or the Coleman index to prevent actions, while they are principally impossible for e.g. the Johnston index.
\end{abstract}

Keywords: simple games, weighted majority games, power indices

MSC: 91B12, 94C10

\section{INTRODUCTION}

Consider a committee that attains its decisions by voting like e.g. the U.S. Senate or the European Parliament. Whenever the committee members have different capabilities to influence decisions, the question for the measurement of individual power arises. Dating back to the late 18th century, Luther Martin was probably the first approaching this issue, see e.g. [30]. Since the second half of the 20th century, several power indices were formally introduced in order to measure the voting power in collective decision making procedures, see e.g. [6, 9, 29, 51, 52] for some surveys and discussions. A huge amount of literature has been devoted to the study of the properties, shortcomings, paradoxes, and axiomatic foundations of several power indices. Within the scientific community the general approval of a unique power index as the optimal compromise is not yet in sight So some researches argue to choose the appropriate index according to the situation?] see e.g. [76].

Having a suitable power index at hand, one can ask the fundamental problem of how to design a voting procedure, such that the resulting distribution of power (according to the chosen power index) among the committee members meets or almost meets a fixed vector ${ }^{3}$ This is commonly called the inverse power index problem ${ }^{7}$ As an algorithmic answer, several heuristics, mostly with an iterative nature, and exact algorithms have been developed, see [48] for a recent overview. The proposed heuristics are generally quite fast and produce good numerical results, meaning that they achieve small approximation errors on practical instances. From the theoretical point of view there is a fundamental problem, since those algorithms are not able to give a priori bounds what approximation errors are achievable. Also non-trivial a posteriori bounds are missing. Thus one simply does not know whether an iterative heuristic has converged sufficiently, i.e. its approximation error is near the minimum possible approximation. Methods based on exhaustive enumeration of voting procedures, see e.g. [19, 20, 45], can in principle determine the exact solution of the inverse problem in finite time. Since for most classes of voting procedures their number grows faster than exponential, those exhaustive algorithms are applicable for small numbers of voters only. Unfortunately, no intermediate lower bounds are produced so that one either has to completely determine the exact solution or

\footnotetext{
${ }^{1}$ Arguably, some power indices, like the Banzhaf and the Shapley-Shubik index, are generally more accepted and applied than others. On the other hand, the pros and cons of several power indices are frequently discussed in the older and latest literature.

${ }^{2}$ As an example, we mention [10] arguing that the Johnston index is best suited for measuring presidential power.

${ }^{3}$ There exists a stream of literature discussing the question of a fair power distribution within a committee, see e.g. [54, 69, 53, 75].

${ }^{4}$ There are several more recent papers using this denomination and trying to algorithmically attack this issue. Considerations about the problem itself date back for a longer time, see. e.g. [41 66, 68].
} 
to be contented with the best solution found in a limited time horizon, i.e. using the approach as an elaborate heuristic. Approaches based on integer linear programming, see [46], automatically come with lower and upper bounds in the intermediate computation steps. The drawback is the lack of theoretical bounds on the running time and the approximation quality, when interrupted after a polynomial running time. Recently, several algorithms have been designed that can achieve a sufficiently accurate approximation with running time polynomial in terms of the number of players times a factor depending on the desired approximation quality, see [17, 67, 18] $]^{5}$

Since the number of voting procedures is generally finite as the number of voters is fixed, one clearly cannot approximate certain power distributions too closely if the number of voters is small. As mentioned before, the number of voting procedures grows faster than exponential for the most relevant classes, so that one might expect that for large committees each desired power distribution can be approximated to a nicety. Indeed there exists a stream of literature concerning with limit results saying that under certain technical conditions one can simply choose the entries of a given power distribution as weights in a weighted voting game, whose power distribution then is relatively close to the initial vector, see e.g. [13, 26, 49, 56, 57, 59, 64, 73].

It was quite a surprise when Alon and Edelman showed that many power distributions, where most of the power is concentrated on a small number of players, are hard to approximate independently of the number of players. In their seminal paper [1], the authors give explicit bounds stating that a Banzhaf vector, whose weight is concentrated on $k<n$ players, has to be near to the Banzhaf vector of a game with $n-k$ null players, which is essentially a game with just $k$ players. In [46], their theorem was applied to deduce that for $n \geq 2$ players the $\|\cdot\|_{1}$-distance between the Banzhaf vector of a simple game and the power distribution $(0.75,0.25,0, \ldots)$ has to be at least $\frac{1}{9}$. Computationally a lower approximation bound of at least 0.37846 was shown for all simple games up to 11 players.

The contribution of this paper consists of a slight tightening of the Alon-Edelman bound. As a consequence, we can deduce that for $n \geq 2$ players the $\|\cdot\|_{1}$-distance between the Banzhaf vector of a simple game and $(0.75,0.25,0, \ldots)$ has to be at least $\frac{1}{8}$. We slightly generalize their theorem so that it is applicable for some classes of voting procedures other than simple games. More importantly, we continue their study of inverse power index problems and prove similar bounds for several power indices defined in the literature. As argued before, this is relevant for the practical application of power indices, since different power indices are suitable in different settings and the Banzhaf index is just a single, while rather important, example of a power index. To this end we break down the approach of Alon and Edelman into smaller pieces, which can be studied from a more general point of view. Several parts of this new theoretic framework can then be used for different power indices and the considerations depending on the precise definition, of the power index under study, are somewhat minimized. It will turn out that many power indices, including e.g. the Public Good index, the Shift index, and the Deegan-Packel index, admit an Alon-Edelman type approximation bound, while the Johnston index does not admit such a result. For other concepts of power indices, like $p$-binomial semivalues, the rounding procedure proposed in [1] does not work directly, but may be adjusted in a meaningful way. So there are classes of power indices that admit Alon-Edelman type results and others that do not. With respect to the classification of power indices and their comparison, this structural distinction might be interesting in itself and deserves further study. While we can decide to which class certain power indices belong for many cases, we leave a few examples, like the ShapleyShubik index, open and just state conjectures.

The rest of the paper is organized as follows. In Section 2 we formally introduce the classes of binary voting procedures along with some notational conventions. The power indices we are studying in this paper are defined in Section 3 . The main general framework for Alon-Edelman type results is stated in Section 4. An integer linear programming formulation for the inverse power index problem for power indices based on counting functions is stated in Section 5. The counterpart of desired power distributions, where most of the power is concentrated on a small number of coordinates, is studied in Section 6. It turns out that with the aid of so-called limit results the (approximate) inverse power index problem becomes almost trivial under certain technical conditions. We end the main part of the paper with a conclusion and some remarks on possible future work in Section 7 . The technical

\footnotetext{
${ }^{5}$ For completeness, we remark that there is also a stream of literature that characterizes the sets of transferable utility games, i.e. more general objects than the voting procedures that we will study here, whose distribution vector of a certain value, i.e. a more general object than a power index, exactly coincides with a given vector, see e.g. [22] 23, 24].
} 
proofs for our Alon-Edelman type results from Section 4 are shifted to Appendix A. The technical details for the parametric class of weighted voting games, e.g. used to prove the non-existence of an Alon-Edelman type result for the Johnston index, are presented in Appendix B.

\section{BINARY VOTING PROCEDURES}

In this paper we restrict ourselves to the study of binary voting procedures. By this we mean that each committee member has the possibility to vote either 'yes' or 'no' for each proposal. The aggregated committee decision then also has to be either 'yes' or 'no'. This assumption is certainly an oversimplification in several practical applications and more general models with several levels of approvals in the input and output have been stated in the literature, see e.g. [8, 34, 35]. Also continuous models or the presence of communication, interaction structures, or a priori unions have been studied so far. We will abstain from all those refinements and leave the study of the corresponding Alon-Edelman type bounds as significant open research problems. Next we will give a brief introduction in the taxonomy of binary voting procedures and refer the interested reader to e.g. [77] for a more detailed treatment.

Within this paper we denote by $N$ the set of committee members, voters, or players. For brevity, we will only use the term players in the following, which is commonly used in the literature. Its cardinality $|N|$ is denoted by $n$. Since in our context the names of the players play no role, we will typically use the set $N=\{1,2, \ldots, n\}=:[n]$. Similarly let $(k, n]:=\{k+1, \ldots, n\}$, for positive integers $k<n$, and $2^{X}$ denote the set of all subsets of a (finite) set $X$. Subsets of $N$, i.e. elements of $2^{N}$, are also called coalitions. To keep notation as simple as possible we will frequently use the following set-theoretic abbreviations: $N-S=N \backslash S, S-i=S \backslash\{i\}, S+i=S \cup i=S \cup\{i\}$, where $S, N$ are sets and $i \in S$.

Definition 2.1. A Boolean game is a function $v: 2^{N} \rightarrow\{0,1\}$ with $v(\emptyset)=0$ and $v(N)=1$. The set of all Boolean games on $n$ players, i.e. one can assume $N=[n]$, is denoted by $\mathcal{B}_{n}$.

The restriction $v(\emptyset)=0$ is some kind of a minimal reasonable assumption in the context of voting in a committee. In a situation where no player is in favor of a proposal it would be pretty factitious if the aggregated overall committee decision would be 'yes'. A similar consideration can be performed for the situation where all committee members are in favor of a proposal. More such reasonability arguments come to one's mind immediately, e.g. an enlarged group of supporters should not turn the aggregated decision from 'yes' to 'no':

Definition 2.2. A simple game is a Boolean game $v: 2^{N} \rightarrow\{0,1\}$ such that $v(S) \leq v(T)$ for all $\emptyset \subseteq S \subseteq T \subseteq N$. The set of all simple games on n players is denoted by $\mathcal{S}_{n}$.

The coalitions $S \subseteq N$ of a Boolean game $v$ with $v(S)=1$ are called winning coalitions and the other ones are called loosing coalitions. If $S$ is a winning coalition, but all of its proper subsets are losing, we call $S$ a minimal winning coalition. Similarly we call a losing coalition $S$ maximal losing if all of its proper supersets are winning.

Definition 2.3. Let $v$ be a Boolean game. By $\mathcal{W}$ we denote the set of all winning and by $\mathcal{W}^{m}$ we denote the set of all minimal winning coalitions of $v$. Similarly, by $\mathcal{L}$ we denote the set of all losing and by $\mathcal{L}^{M}$ we denote the set of all maximal losing coalitions of $v$.

We remark that each simple game is uniquely characterized by specifying $N$ and either one of the sets $\mathcal{W}, \mathcal{W}^{m}, \mathcal{L}$, or $\mathcal{L}^{M}$. So in the following we will from time to time also use the pair $(\mathcal{W}, N)$ to denote a simple game instead of the function notation $v: 2^{N} \rightarrow\{0,1\}$. A player that is not contained in any minimal winning coalition is called a null player. Boolean games are uniquely characterized by either of the sets $\mathcal{W}$ or $\mathcal{L}$.

Logically inverting the statement of a proposal can result in counterintuitive outcomes in the context of voting, if all simple games are permitted. So far it is possible that both, a coalition $S$ and its complementary coalition $N \backslash S$, can carry through a proposal.

Definition 2.4. A simple game is called proper if the complement $N-S$ of any winning coalition $S$ is losing. It is called strong if the complement $N-S$ of any losing coalition $S$ is winning. A simple game that is both proper and strong is called constant-sum (or self-dual, or decisive). 
The desirability relation, introduced in [42], assumes a certain intuitive ordering of the players:

Definition 2.5. Given a simple game $(\mathcal{W}, N)$ we say that player $i \in N$ is more desirable as player $j \in$ $N$, denoted by $i \succeq j$, if

(1) for all $S \subseteq N-\{i, j\}$ with $S+j \in \mathcal{W}$, we have $S+i \in \mathcal{W}$;

(2) for all $S \subseteq N-\{i, j\}$ with $S+i \in \mathcal{L}$, we have $S+j \in \mathcal{L}=2^{N}-\mathcal{W}$.

We write $i \simeq j$ if $i \succeq j, j \succeq i$ and use $i \succ j$ as abbreviation for $i \succeq j, i \not z$.

Definition 2.6. A simple game $(\mathcal{W}, N)$ is called complete if for each pair of players $i, j \in N$ we have $i \succeq j$ or $j \succeq i$. The set of all complete (simple) games on $n$ players is denoted by $\mathcal{C}_{n}$.

Definition 2.7. Let $(\mathcal{W},[n])$ be a complete simple game, where $1 \succeq 2 \succeq \cdots \succeq n$, and $S \subseteq N$. A coalition $T \subseteq N$ is a direct left-shift of $S$ whenever there exists a player $i \in S$ with $i-1 \notin S$ such that $T=S-\{i\}+\{i-1\}$ for $i>1$ or $T=S+\{n\}$ for $n \notin S$. Similarly, a coalition $T \subseteq N$ is a direct right-shift of $S$ whenever there exists a player $i \in S$ with $i+1 \notin S$ such that $T=S-\{i\}+\{i+1\}$ for $i<n$ or $T=S-\{n\}$ for $n \in S$. A coalition $T$ is a left-shift of $S$ if it arises as a sequence of direct left-shifts. For brevity we denote this case by $T \succeq S$. Similarly, it is a right-shift of $S$ if it arises as a sequence of direct right-shifts. For brevity we denote this case by $T \preceq S$. A winning coalition $S$ such that all right-shifts of $S$ are losing is called shift-minimal (winning). Similarly, a losing coalition $S$ such that all left-shifts of $S$ are winning is called shiftmaximal (losing). By $\mathcal{W}^{\text {sm }}$ we denote the set of all shift-minimal winning coalitions of $(\mathcal{W}, N)$ and by $\mathcal{L}^{s M}$ the set of all shift-maximal losing coalitions.

We remark that every shift-minimal winning coalition has to be a minimal winning coalition and each shift-maximal losing coalition has to be a maximal losing coalition.

Definition 2.8. A simple game $(\mathcal{W},[n])$ is weighted if there exists a quota $q>0$ and weights $w_{1}, \ldots, w_{n} \geq 0$ such that $S$ is winning if and only if $w(S)=\sum_{i \in S} w_{i} \geq q$. We denote the game by $\left[q ; w_{1}, \ldots, w_{n}\right]$. The set of all weighted (simple) games on $n$ players is denoted by $\mathcal{T}_{n}$.

Since the weights clearly induce the desirability relation, each weighted game is complete. We remark that weighted representations are not unique, e.g. $[2 ; 2,1,1]=[3 ; 3,2,1]$. Not every simple game is weighted, but it can be represented as a finite intersection of weighted games.

The number of Boolean games on $n$ players, not taking symmetry into account, is clearly given by $2^{2^{n}-1}$. Enumeration results for the other subclasses of binary voting procedures can be found in [20, 45, 50].

\section{POWER INDICES}

Power indices are a formal way to measure the influence of a single player on the outcome of a committee decision. They became necessary when researchers relatively early have discovered that the relative influences are not directly proportional to voting weights in a weighted game, see e.g. [48] for a more extensive discussion. In most cases, power indices can be defined for larger classes than weighted games, sometimes even for transferable utility games. Many power indices have been proposed so far in the literature. Here, we want to briefly present a large collection of those and arrange them in a somewhat systematic order. Our classification and listing is based on [3, 6, 9, 29, 30, 38, 51, 52, 65 6 . In general terms, a power index on a class $\mathcal{V}_{n} \subseteq \mathcal{B}_{n}$ of binary games, consisting of $n$ players, is a mapping $g: \mathcal{V}_{n} \rightarrow \mathbb{R}^{n}$. Mostly we use $\mathcal{V}_{n} \in\left\{\mathcal{B}_{n}, \mathcal{S}_{n}\right\}$ and in some cases the restriction to $\mathcal{V}_{n} \in\left\{\mathcal{C}_{n}, \mathcal{T}_{n}\right\}$. Usually we state the definition of the vector-valued power indices just for its components $g_{i}$, i.e. for an arbitrary player $i \in N$.

3.1. Power indices derived from values. Some power indices are derived from values or solution concepts for transferable utility games, see e.g. [4, 12] for the more general point of view. Here we will state only the restriction of the underlying values to power indices, i.e. to simple or Boolean games instead of transferable utility games. Let us start with one of the most famous values or power indices: In [74], the Shapley value was axiomatically introduced and shortly after, see [75], restricted to simple games:

\footnotetext{
${ }^{6}$ Also the website http://powerslave.val.utu.fi/indices.html has served as a source.
} 
Definition 3.1. The Shapley-Shubik index of a Boolean game $v: 2^{N} \rightarrow\{0,1\}$ for voter $i \in N$ is given by

$$
\operatorname{SSI}_{i}(v)=\sum_{S \subseteq N-i} \frac{|S| !(|N|-1-|S|) !}{|N| !} \cdot(v(S \cup i)-v(S)) .
$$

The Shapley-Shubik index satisfies some nice properties, i.e. it is symmetric, positive, efficient on $\mathcal{S}_{n}$ and satisfies the null player property.

Definition 3.2. Let $g: \mathcal{V}_{n} \rightarrow \mathbb{R}^{n}=\left(g_{i}\right)_{i \in[n]}$ be a power index on a class $\mathcal{V}_{n}$ of binary games. We say that

(1) $g$ is symmetric: if for all $v \in \mathcal{V}_{n}$ and any bijection $\tau:[n] \rightarrow[n]$ we have $g_{\tau(i)}(\tau v)=g_{i}(v)$, where $\tau v(S)=v(\tau(S))$ for any coalition $S \subseteq[n]$;

(2) $g$ is positive: if for all $v \in \mathcal{V}_{n}$ and all $i \in[n]$ we have $g_{i}(v) \geq 0$ and $g(v) \neq 0$;

(3) $g$ is efficient: if for all $v \in \mathcal{V}_{n}$ we have $\sum_{i=1}^{n} g_{i}(v)=1$;

(4) $g$ satisfies the null player property: if for all $v \in \mathcal{V}_{n}$ and all null players $i$ of $v$ we have $g_{i}(v)=0$.

We remark that the Shapley-Shubik index is not efficient on $\mathcal{B}_{n}$ in general (for $n \geq 3$ ) by looking at the following example: Define the Boolean game $v: 2^{[3]} \rightarrow\{0,1\}$, which attains the value 1 exactly for the coalitions $\{1\},\{2\}$, and $\{1,2,3\}$. Inserting into the definition gives $\operatorname{SSI}_{1}(v)=\operatorname{SSI}_{2}(v)=\frac{1}{2}$, and $\operatorname{SSI}_{3}(v)=\frac{1}{3}$, which sums up to $\frac{4}{3} \neq 1$. Nevertheless, Boolean games might be seen as a rather obscure class of binary voting procedures, we remark that many of the subsequent power indices are not efficient. Since efficiency is a desirable property (in some contexts), we generally define a normalization of a power index in order to make it efficient:

Definition 3.3. For a positive power index $P: \mathcal{V}_{n} \rightarrow \mathbb{R}^{n}$ we define the corresponding normalized power index $\widehat{P}: \mathcal{V}_{n} \rightarrow \mathbb{R}^{n}$ as $\widehat{P}_{i}(v)=\frac{P_{i}(v)}{\sum_{j=1}^{n} P_{j}(v)}$ for all $v \in \mathcal{V}_{n}$.

Lemma 3.4. With the notation and assumptions from Definition $3.3 . \widehat{P}$ is efficient (on $\mathcal{V}_{n}$ ).

If we call each player $i \in N$ a vetoer of a simple game $v$ whenever $v(N-i)=0$, then we can restate the index from [78]:

Definition 3.5. The Tijs index of a simple game $v: 2^{N} \rightarrow\{0,1\}$, containing at least one vetoer, for player $i \in N$ is given by $\operatorname{Tijs}_{i}(v)=1$ is $i$ is a vetoer and zero otherwise.

The Tijs index is symmetric and satisfies the null player property. If the class of games is restricted to those containing at least one vetoer, the Tijs index is also positive, so that its normalized version is efficient due to Lemma 3.4. For our purposes the possibility Tijs $(v)=0$ causes minor technical difficulties. Nevertheless vetoers are an important concept in political science. In [79] analyses of existing political institutions have been formalized into the so-called veto-player-theorem, e.g. stating that departure from the status quo in political institutions is more likely, the smaller is the number of vetoers (which makes also sense if no vetoer is present).

In [72], the author has introduced the nucleolus Nuc as the lexicographically minimal imputation. It has been proposed as a power index as recently as the last decade, see e.g. [54, 60, 61]. We remark that the nucleolus satisfies all four properties of Definition 3.2 (on Boolean games).

Semivalues were introduced in [80] for simple games and later generalized in [25]. They can be seen as weighted averages of a player's marginal contribution to coalitions.

Definition 3.6. Let $\mathbf{p}=\left(p_{0}, \ldots, p_{n-1}\right)$ be a vector of non-negative real numbers satisfying $\sum_{j=0}^{n-1} p_{j}\left(\begin{array}{c}n-1 \\ j\end{array}\right)=$ 1. The semivalue of a Boolean game $v: 2^{N} \rightarrow\{0,1\}$ with respect to $\mathbf{p}$ for player $i \in N$ is given by

$$
\Psi_{i}^{\mathbf{p}}(v)=\sum_{S \subseteq N-i} p_{|S|} \cdot[v(S \cup i)-v(S)] .
$$

The Shapley-Shubik index is given by $p_{j}=\frac{1}{n \cdot\left(\begin{array}{c}n-1 \\ j\end{array}\right)}$ and the later on defined absolute Banzhaf index is given by $p_{j}=\frac{1}{2}$ for all $0 \leq j \leq n-1$. Semivalues are symmetric, positive and satisfy the null player property on $\mathcal{S}_{n}$, but typically are not efficient.

Definition 3.7. For $p \in(0,1)$ the semivalue $\Psi^{p}=\Psi^{\mathbf{p}}$, with $p_{j}=p^{j}(1-p)^{n-1-j}$ for $0 \leq j \leq n-1$, is called a $p$-binomial semivalue. 
3.2. Power indices bases on winning, swing, and critical coalitions. In [6] the authors present a large list of autonomously generated power indices, as they call them, which are, in contrast to those from the previous section, not obtained from values. We repeat and enlarge their list at this place and the following subsection. All examples are based on winning, critical, minimal winning, shiftminimal winning, and swing coalitions and in some sense more or less related to the Banzhaf index. A possibly more general point of view is taken in Subsection 3.5 .

In order to state the definitions of the announced power indices we need a bit more notation:

Definition 3.8. Let $v=(\mathcal{W}, N)$ be a simple game. A coalition $S \subseteq N-i$ with $v(S \cup i)-v(S)=1$ is called an $i$-swing or a swing for player $i$. The number of $i$-swings of player $i \in N$ is denoted by $\eta_{i}$. As abbreviation we use $\eta=\sum_{j=1}^{n} \eta_{j}$.

Let $\mathcal{W}_{i}$ denote the set of winning coalitions that contain player $i$. For an $i$-swing $S \subseteq N-i$ the coalition $S \cup i$ is a winning coalition. We say that $i$ is critical in $S \cup i$ since removing player $i$ from $S \cup i$ turns the coalition from winning to losing. For a losing coalition $S \subseteq N-i$, we similarly say that player $i$ is critical if $S \cup i$ is winning.

Definition 3.9. The absolute Banzhaf index $]^{7}$ of a simple game $v=(\mathcal{W},[n])$ for player $i \in N$ is given by $\mathrm{BZ}_{i}(v)=\eta_{i}(v) / 2^{n-1}$.

Its normalization $\widehat{\mathrm{BZ}}$ is usually called the relative Banzhaf index and satisfies all four properties of Definition 3.2.

In [15] the author, among other things, defined two power indices:

Definition 3.10. Let $v: 2^{N} \rightarrow\{0,1\}$ be a simple game. The Coleman power of a member to prevent action for player $i \in N$ is given by

$$
\operatorname{ColPrev}_{i}(v)=\frac{\# \text { winning coalitions in which } i \text { is critical }}{\text { total number of winning coalitions }}=\frac{\eta_{i}(v)}{|\mathcal{W}|}
$$

and the Coleman power of a member to initiate action is given by

$$
\operatorname{ColIni}_{i}(v)=\frac{\# \text { losing coalitions in which } i \text { is critical }}{\text { total number of losing coalitions }} .
$$

As noted e.g. in [26], the normalized versions of the power indices from Definition 3.9 and Definition 3.10 coincide, i.e. we have $\widehat{\mathrm{BZ}}=\widehat{\mathrm{ColPrev}}=\widehat{\mathrm{ColIni}}$. In other words those three power indices are scaled versions of each other. We remark that for different purposes normalizing power indices indeed destroys some information since the scaling variant depends non-trivially on the game. So in practice all three power indices may have their justification.

Definition 3.11. The Rae index ${ }^{8}$ of a Boolean game $v=2^{N} \rightarrow\{0,1\}$ for player $i \in N$ is given by

$$
\operatorname{Rae}_{i}(v)=\frac{|S \subseteq N: i \in S, v(S)=1|+|S \subseteq N: i \notin S, v(S)=0|}{2^{|N|}} .
$$

In [26, Eq. 53, p. 124], the authors prove the identity

$$
\operatorname{Rae}(v)=\frac{1}{2}+\frac{1}{2} \cdot \operatorname{Bz}(v)
$$

which was also known to Penrose long before the publication of Rae, see e.g. [30]. In other words, the Rae index arises as a linear transformation from the absolute Banzhaf index. In our context we can easily conclude Alon-Edelman type bounds for linearly transformed power indices, see Lemma 4.12 and Lemma 4.18 .

Definition 3.12. The König-Bräuninger index 9 of a Boolean game $v=2^{N} \rightarrow\{0,1\}$ for player $i \in N$ is given by

$$
\mathrm{KB}_{i}(v)=\frac{\# \text { winning coalitions that contain } i}{\text { total number of winning coalitions }}=\frac{|\mathcal{W}|}{|\mathcal{W}|}
$$

\footnotetext{
${ }^{7}$ Named after [5], but originally going back to [69], so that some authors speak of the Penrose-Banzhaf index.

${ }^{8}$ Originally, the Rae index was introduced in [70]. It coincides with the satisfaction index studied in a different context in [11] and is sometimes called the Brams-Lake index.

${ }^{9}$ Introduced in [44] and also called inclusiveness index. This index is equivalent to the Zipke index, see [63].
} 
A rather similar power index has been defined in [7]:

Definition 3.13. The Public Help index of a Boolean game $v=2^{N} \rightarrow\{0,1\}$ for player $i \in N$ is given by

$$
\operatorname{PHI}_{i}(v)=\frac{\left|\mathcal{W}_{i}\right|}{\sum_{j \in N}\left|\mathcal{W}_{j}\right|} .
$$

The last two power indices are closely related to the so-called Chow parameters introduced in [14]. In their original form, they are given by the $n+1$ numbers $\left|\mathcal{W}_{1}\right|, \ldots,\left|\mathcal{W}_{n}\right|$ and $\left.|\mathcal{W}|\right|^{10}$ We remark that the (original) Chow parameters uniquely characterize each simple game. Dropping the number of winning coalitions gives a vector of $n$ numbers, which uniquely characterizes each weighted game, and can be used as a power index. We have $\widehat{\mathrm{KB}}=\widehat{\mathrm{PHI}}=\widehat{\mathrm{Chow}}$ and remark that the vector $\left(\eta_{1}, \ldots, \eta_{n}\right)$ of the number of swings is called Banzhaf score by some authors.

Definition 3.14. The Chow index of a Boolean game $v=2^{N} \rightarrow\{0,1\}$ for player $i \in N$ is given by

$$
\operatorname{Chow}_{i}(v)=\left|\mathcal{W}_{i}\right| \text {. }
$$

The power indices described so far in this subsection, are all linear transforms of counting winning, losing, swing, or critical coalitions. Here, an object like a winning coalition for the Chow index can be counted for multiple players, i.e. it is counted for all members of the respective coalition. Another concept is to distribute the contribution, of a winning coalition $S$ in our example, equally among the contributing players, i.e. to just count $\frac{1}{|S|}$ instead of 1 for all members of $S$. In Subsection 3.5. or more precisely in Definition 3.22, we describe the underlying idea in more detail. Applying this rather general concept to the absolute Banzhaf index after multiplication with $2^{n-1}$ gives:

Definition 3.15. The absolute Johnston index ${ }^{11}$ of a Boolean game $v=2^{N} \rightarrow\{0,1\}$ for voter $i \in N$ is given by

$$
\operatorname{JS}_{i}(v)=\sum_{\{i\} \subseteq S \subseteq N: i \text { is critical in } S, v(i)=1} \frac{1}{\# \text { of critical players in } S} .
$$

For the weighted game $[2 ; 2,1,1]$ with player set [3], the absolute Johnston index is given by $\left(3, \frac{1}{2}, \frac{1}{2}\right)$. Its normalization $\widehat{\mathrm{JS}}$, called relative Johnston index, is given by $\left(\frac{3}{4}, \frac{1}{8}, \frac{1}{8}\right)$. For the weighted voting game $[3 ; 2,1,1]$ with player set $[3]$ we have $\mathrm{JS}=\left(2, \frac{1}{2}, \frac{1}{2}\right)$ and $\widehat{\mathrm{JS}}=\left(\frac{2}{3}, \frac{1}{6}, \frac{1}{6}\right)$, which coincides with the Shapley-Shubik vector.

3.3. Power indices derived from minimal or shift-minimal winning coalitions. In the previous subsection we have considered power indices based on winning, critical, or swing coalitions. The underlying idea is that the distinction between a winning and a losing coalition is the crucial difference which should be mirrored in the definition of a power index. On the other hand, Riker introduced so the so-called Riker's size principle in [71], claiming that parties attempt to increase the size of a coalition supporting a proposal only until the point where it gets minimally winning. Thus, we consider power indices based on minimal or shift-minimal winning coalitions in this subsection and remark that there are also other lines of argumentation in order to justify those concepts, see e.g. [40, 39, 81]. As abbreviation we use $\mathcal{W}_{i}^{m}$ for the set of minimal winning coalitions containing player $i$ and $\mathcal{W}_{i}^{s m}$ for the set of shift-minimal winning coalitions containing player $i$.

Definition 3.16. The absolute Public Good index ${ }^{12}$ of a simple game $v=2^{N} \rightarrow\{0,1\}=(\mathcal{W}, N)$ for voter $i \in N$ is given by

$$
\operatorname{PGI}_{i}(v)=\left|\mathcal{W}_{i}^{m}\right| \text {. }
$$

Its normalization $\widehat{\mathrm{PGI}}$ is called (relative) Public Good index.

As the Johnston index arises from the Banzhaf index by a certain payoff-distribution rule, see Definition 3.22, there is also a counterpart to the Public Good index:

\footnotetext{
${ }^{10}$ Some authors have redefined them as $\eta_{1}, \ldots, \eta_{n}$, i.e. the number of $i$-swings being the numerators of the absolute Banzhaf index, and $|\mathcal{W}|$. The relation between both versions is given by $\eta_{i}=2\left|\mathcal{W}_{i}\right|-|\mathcal{W}|$, see e.g. [26].

${ }^{11}$ Introduced in [43] and also called Johnston score by some authors.

${ }^{12}$ The (relative) Public Good index was introduced in [38] and is also known as the Holler-Packel index due to an axiomatization of Holler and Packel.
} 
Definition 3.17. The absolute Deegan-Packel index ${ }^{13}$ of a simple game $v=2^{N} \rightarrow\{0,1\}=(\mathcal{W}, N)$ for player $i \in N$ is given by

$$
\operatorname{DP}_{i}(v)=\sum_{S \in \mathcal{W}_{i}^{m}} \frac{1}{|S|} .
$$

Its normalization $\widehat{\mathrm{DP}}=\frac{1}{\left|\mathcal{W}^{m}\right|} \cdot \mathrm{DP}$ is called (relative) Deegan-Packel index.

For shift-minimal winning coalitions we have a similar pair of power indices:

Definition 3.18. The absolute Shift index ${ }^{14}$ of a complete simple game $v=2^{N} \rightarrow\{0,1\}=(\mathcal{W}, N)$ for player $i \in N$ is given by

$$
\operatorname{Shift}_{i}(v)=\left|\mathcal{W}_{i}^{s m}\right| \text {. }
$$

Its normalization $\widehat{\text { Shift }}$ is called (relative) Shift index.

Definition 3.19. The absolute Shift-Deegan-Packel index $\left[15\right.$ of a complete simple game $v=2^{N} \rightarrow$ $\{0,1\}=(\mathcal{W}, N)$ for player $i \in N$ is given by

$$
\operatorname{SDP}_{i}(v)=\sum_{S \in \mathcal{W}_{i}^{s m}} \frac{1}{|S|} .
$$

Its normalization $\widehat{\mathrm{SDP}}=\frac{1}{\left|\mathcal{W}^{s m}\right|} \cdot \mathrm{SDP}$ is called (relative) Shift-Deegan-Packel index.

3.4. Power indices for weighted games. Most of the power indices introduced so far were defined for simple games or even Boolean games. The Shift index and the Shift-Deegan-Packel index from the previous subsection are an exception that can only be defined for complete simple games. For the most restrictive class of binary voting procedures that we are considering in this paper, i.e. weighted games, also a very few power indices have been introduced in the literature.

In [16] the authors defined a different variant of the Banzhaf index by reweighting a swing based counting. For a given weighted voting game $v: 2^{[n]} \rightarrow\{0,1\}=\left[q ; w_{1}, \ldots, w_{n}\right]$ the Colomer index, which is also known under the name executive power index, is defined as

$$
\operatorname{Colomer}_{i}(v)=\frac{\sum_{\{i\} \subseteq S \subseteq N} \frac{w_{i} \cdot(v(S)-v(S-i))}{\sum_{j \in S} w_{j} \cdot(v(S)-v(S-j))}}{\sum_{S \subseteq N} f(S)}=\frac{\sum_{S \in \mathcal{W}} \frac{w_{i} \cdot(v(S)-v(S-i))}{\sum_{j \in S} w_{j} \cdot(v(S)-v(S-j))}}{|\mathcal{W}|} .
$$

We remark that this index directly depends on the weighted representation of the game and not only the underlying simple game, i.e. the function $v$ does not suffices to determine the values of the power distribution, so that our notation $\operatorname{Colomer}_{i}(v)$ is slightly misleading.

An index that is not harmed by the ambiguity of weighted representations is defined in [31]. The underlying theoretical concepts are minimum sum integer representations of weighted games, see e.g. [45, 33]. While such representations do not need to be unique in general, at the very least the set of such distinguished representations is finite in all cases. The so-called MSR index is defined as the average over all minimum sum integer representations of weighted game. In some sense this index brings us back to the original motivation for power indices, i.e. to have a measure for influence better than the original weights, which can vary to a large extend of what is generally considered as being meaningful.

3.5. Counting functions. Many of the power indices defined in the previous subsections have a common structure, i.e. they arise by counting a certain quantity like swing coalitions or winning coalitions ${ }^{16}$. In some cases these counts are weighted like for the $p$-binomial semivalues. Almost always those counts can be decomposed as a sum over all coalitions.

\footnotetext{
${ }^{13}$ The (relative) Deegan-Packel index was introduced in [21].

${ }^{14}$ The (relative) Shift index was introduced in [2]. As a justification for power indices based on shift-minimal winning coalitions one may go along Riker's size principle and additionally assume that parties try to avoid the inclusion of powerful members.

${ }^{15}$ The (relative) Shift-Deegan-Packel index was introduced in [3].

${ }^{16}$ Several authors have tried to provide a description of such a common structure, see e.g. [6, 58]. Our approach does not claim to be superior and has of course many similarities, but it seems to be more convenient in our situation.
} 
Definition 3.20. Let $\mathcal{V}_{n}$ be a class of binary games on $n$ players. A counting function $C\left(\right.$ on $\left.\mathcal{V}_{n}\right)$ is a mapping from $\mathcal{V}_{n} \times 2^{N} \times N$ to $\mathbb{R}_{\geq 0}^{n}$. We write $C_{i}: \mathcal{V}_{n} \times 2^{N} \rightarrow \mathbb{R}_{\geq 0}$ for the restriction of $C$ to an arbitrary player $i \in N$ and $\bar{C}: \mathcal{V}_{n} \times 2^{N} \rightarrow \mathbb{R}_{\geq 0}$ with $\bar{C}(v, S)=\sum_{j \in N} C_{i}(v, S)$.

An example of a counting function, on the set $\mathcal{S}_{n}$ of simple games consisting of $n$ players, is given by

$$
C_{i}(v, S)=\left\{\begin{array}{rll}
1 / 2^{n-1} & : & i \in S, v(S)=1, v(S-i)=0 \\
0 & : & \text { otherwise. }
\end{array}\right.
$$

We remark that it counts the number $\eta_{i}$ of $i$-swings via $\eta_{i}(v)=2^{n-1} \cdot \sum_{S \subseteq N} C_{i}(v, S)$. A different counting function that counts the same quantities is given by

$$
\widetilde{C}_{i}(v, S)=\left\{\begin{array}{rll}
1 / 2^{n-1} & : & i \notin S, v(S)=0, v(S+i)=1, \\
0 & : & \text { otherwise. }
\end{array}\right.
$$

Definition 3.21. Let $\mathcal{V}_{n}$ be a class of binary games on $n$ players. Given a counting function $C$ on $\mathcal{V}_{n}$, the induced power index $P: \mathcal{V}_{n} \rightarrow \mathbb{R}_{\geq 0}^{n}\left(\right.$ on $\left.\mathcal{V}_{n}\right)$ is given by

$$
P_{i}(v)=C_{i}\left(v, 2^{N}\right):=\sum_{S \in 2^{N}} C_{i}(v, S)
$$

for all $i \in[n]$ and all $v \in \mathcal{V}_{n}$. By $\hat{P}$ we denote the normalized version of $P$, see Definition 3.3

The induced power indices of both $C$ and $\widetilde{C}$, as stated above, are equivalent to the absolute Banzhaf index. In the following we will write $C^{\mathrm{Bz}}$ when referring to this counting function $C$. When summed up over all coalitions $C_{i}^{\mathrm{Bz}}$ counts the number of coalitions where $i$ is a critical player. Similarly $\sum_{i=1}^{n} \sum_{S \subseteq N} C_{i}^{\mathrm{Bz}}(\cdot, S)$ gives the number of coalitions with at least a critical player, including multiplicities.

We remark that it is not too hard to give counting functions for all power indices of Section 3 , except for the nucleolus, the Colomer index and the MSR index, such that the corresponding induced power index is equivalent to the respective power index. Two such examples are given by

$$
C_{i}^{\mathrm{PGI}}(v, S)=\left\{\begin{array}{lll}
1 & : & S \in \mathcal{W}_{i}^{m} \\
0 & : & \text { otherwise }
\end{array}\right.
$$

for the absolute Public Good index and

$$
C_{i}^{\text {Shift }}(v, S)=\left\{\begin{array}{lll}
1 & : & S \in \mathcal{W}_{i}^{s m} \\
0 & : & \text { otherwise }
\end{array}\right.
$$

for the absolute Shift index. The first function counts minimal winning coalitions and the second counts shift-minimal winning coalitions.

Of course it is easily possible to write down a counting function such that the induced power index is equivalent to the absolute Johnston index:

$$
C_{i}^{\mathrm{JS}}(v, S)=\left\{\begin{array}{rll}
\frac{1}{|\{j \in S: v(S-j)=0\}|} & : & i \in S, v(S)=1, v(S-i)=0, \\
0 & : & \text { otherwise. }
\end{array}\right.
$$

But as we mentioned in Subsection 3.2 a more general concept is underlying:

Definition 3.22. Let $\mathcal{V}_{n}$ be a class of binary games on $n$ players and $C$ be a counting function on $\mathcal{V}_{n}$. The equal division counting function $C^{\prime}$ is given by

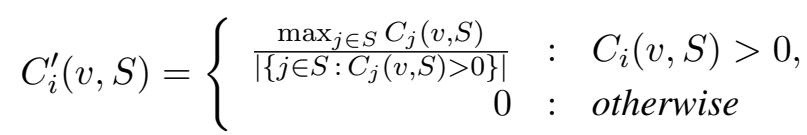

for all $i \in[n]$ and all $v \in \mathcal{V}_{n}$.

In other words, we have $C_{i}^{\prime}(v, S)=C_{j}^{\prime}(v, S)$ for all $i, j \in[n]$ with $C_{i}(v, S), C_{j}(v, S)>0$, i.e. an equal division of the payoff $\max _{j \in S} C_{j}(v, S)$ of coalition $S$ to all contributing players. With this terminology at hand we can state that the counting function of the absolute Johnston index $C^{\mathrm{JS}}$ arises as the equal division version of $2^{n-1} \cdot C^{\mathrm{Bz}}$. This correspondence is the reason why we have defined $C^{\mathrm{Bz}}$ as $C$ and not as $\widetilde{C}$, see the equations before Definition 3.21. We have that $\sum_{i=1}^{n} \sum_{S \subset N} C^{\mathrm{JS}}(v, S)$ equals the number of winning coalitions with at least one critical player (without multiplicities). 
Similarly, the counting function of the absolute Deegan-Packel index $C^{\mathrm{DP}}$ arises as the equal division version of $C^{\mathrm{PGI}}$ and the counting function of the absolute Shift-Deegan-Packel index $C^{\mathrm{SDP}}$ arises as the equal division version of $C^{\text {Shift }}$.

Directly from the definition of a counting function and the definition from a normalized power index we conclude:

Lemma 3.23. Let $\mathcal{V}_{n}$ be a class of binary games on $n$ players, $C$ be a counting function on $\mathcal{V}_{n}$, and $P: \mathcal{V}_{n} \rightarrow \mathbb{R}_{\geq 0}^{n}$ be the induced power index. Then the normalized power index $\widehat{P}$ is induced by the counting function $\widehat{C}=\frac{1}{\Lambda} \cdot C$, where

$$
\lambda(v)=\sum_{i=1}^{n} \sum_{S \subseteq N} C_{i}(v, S)
$$

for each $v \in \mathcal{V}_{n}$, i.e. the scaling factor $\frac{1}{\Lambda}$ may depend on the game $v$.

\section{Alon-Edelman type bounds}

In this section we establish a list of what we call Alon-Edelman type bounds or results for the $\|\cdot\|_{1}$-distance between Boolean games and somewhat simplified or reduced Boolean games. We start with the original result from [1] (slightly rewritten in our notation). To this end, we call a Boolean game $v: 2^{[n]} \rightarrow\{0,1\}$ on $n$ players $k$-pure if all players in $(k, n]$ are null players.

theorem 4.1. (Alon and Edelman, 2010) Let $n>k$ be positive integers, let $\varepsilon<\frac{1}{k+1}$ be a positive real, and let $v=(\mathcal{W},[n])$ be a simple game on $n$ players. If $\sum_{i=k+1}^{n} \widehat{\mathrm{Bz}}_{i}(v) \leq \varepsilon$, then there exists a $k$-pure simple game $v^{\prime}$ on $n$ players so that

$$
\left\|\widehat{\mathrm{Bz}}\left(v^{\prime}\right)-\widehat{\mathrm{Bz}}(v)\right\|_{1}=\sum_{i=1}^{n}\left|\widehat{\mathrm{Bz}}_{i}\left(v^{\prime}\right)-\widehat{\mathrm{Bz}}_{i}(v)\right| \leq \frac{(2 k+1) \varepsilon}{1-(k+1) \varepsilon}+\varepsilon .
$$

In the following we will generalize this theorem for other power indices besides the (relative) Banzhaf index. As a side effect, we will tighten the stated bound to a nicer expression. We go along the lines of the original proof and generalize the underlying ideas. To this end, we separate smaller parts and introduce additional notation aiming at a general framework for Alon-Edelman type results.

Instead of the (relative) Banzhaf index we want to use an almost arbitrary power index, which then of course has to satisfy some technical conditions, since there are power indices for which a AlonEdelman type bound cannot exist (in the precise formulation that we will state shortly), as we will see later on. To condense the mentioned technical conditions we introduce the property of being locally approximable of a power index based on counting functions in Definition 4.11. The quality of the local approximation is quantified with the aid of two functions, which in turn determine the bounds of the Alon-Edelman type results. We will specify how the simplified $k$-pure game $v^{\prime}$ arises from $v$, i.e. we make the existence result constructive ${ }^{17}$, see Definition 4.7 .

Anticipating the necessary notation given in Subsection 4.1, we can state our main theorem as follows:

theorem 4.2. Let $0<k<n$ be integers, $P$ be a power index induced by a counting function $C$ that is locally approximable for the shortening function $\Gamma: \mathcal{V}_{n} \rightarrow \mathcal{V}_{n}$ with quality functions $f_{1}$ and $f_{2}$. If $P$ further satisfies the null player property and is positive ${ }^{18}$ then for each game $v \in \mathcal{V}_{n}$ and its shortening $v^{\prime}=\Gamma(v) \in \mathcal{V}_{n}$ with $\sum_{i=k+1}^{n} P_{i}(v) \leq \varepsilon \cdot \sum_{i=1}^{n} P_{i}(v)$ we have

$$
\left\|P\left(v^{\prime}\right)-P(v)\right\|_{1}=\sum_{i=1}^{k}\left|P_{i}\left(v^{\prime}\right)-P_{i}(v)\right|+\sum_{i=k+1}^{n}\left|P_{i}(v)\right| \leq\left(k f_{1}(k)+1\right) \cdot \varepsilon \cdot \sum_{i=1}^{n} P_{i}(v) .
$$

\footnotetext{
${ }^{17}$ This constructive reformulation was already implicitly contained in the proof of [1].

${ }^{18}$ see Definition 3.2 (4) and Definition 3.2 (2)
} 
If $\sum_{i=k+1}^{n} \widehat{P}_{i}(v) \leq \varepsilon^{\prime}$, then we have

$$
\left\|\widehat{P}\left(v^{\prime}\right)-\widehat{P}(v)\right\|_{1}=\sum_{i=1}^{k}\left|\widehat{P}_{i}\left(v^{\prime}\right)-\widehat{P}_{i}(v)\right|+\sum_{i=k+1}^{n}\left|\widehat{P}_{i}(v)\right| \leq\left(f_{2}(k)+k f_{1}(k)+1\right) \varepsilon^{\prime}
$$

for the normalized power index $\widehat{P}$.

Proof. Since $P_{i}(v)=C_{i}\left(v, 2^{N}\right)$ the condition $\sum_{i=k+1}^{n} P_{i}(v) \leq \varepsilon \cdot \sum_{i=1}^{n} P_{i}(v)$ can be rewritten as $\sum_{i=k+1}^{n} C_{i}\left(v, 2^{N}\right) \leq \varepsilon \cdot \bar{C}\left(v, 2^{N}\right)$. From local approximability we conclude

$$
\sum_{i=1}^{k}\left|P_{i}\left(v^{\prime}\right)-P_{i}(v)\right|=\sum_{i=1}^{k}\left|C_{i}\left(v^{\prime}, 2^{N}\right)-C_{i}\left(v, 2^{N}\right)\right| \leq k \cdot f_{1}(k) \cdot \varepsilon \cdot \bar{C}\left(v, 2^{N}\right),
$$

where the ride hand side equals $k f_{1}(k) \cdot \varepsilon \cdot \sum_{i=1}^{n} P_{i}(v)$. Since $P$ is positive we have $\sum_{i=k+1}^{n}\left|P_{i}(v)\right|=$ $\sum_{i=k+1}^{n} P_{i}(v) \leq \varepsilon \cdot \sum_{i=1}^{n} P_{i}(v)$, so that we can combine both inequalities to obtain the proposed bound for $\left\|P\left(v^{\prime}\right)-P(v)\right\|_{1}$.

$$
\begin{aligned}
& \left\|\widehat{P}\left(v^{\prime}\right)-\widehat{P}(v)\right\|_{1}=\sum_{i=1}^{k}\left|\frac{C_{i}\left(v, 2^{N}\right)}{\bar{C}\left(v, 2^{N}\right)}-\frac{C_{i}\left(v^{\prime}, 2^{N}\right)}{\bar{C}\left(v^{\prime}, 2^{N}\right)}\right|+\sum_{i=k+1}^{n} \widehat{P}_{i}(v) \\
\leq & \sum_{i=1}^{k}\left|\frac{C_{i}\left(v, 2^{N}\right)}{\bar{C}\left(v, 2^{N}\right)}-\frac{C_{i}\left(v^{\prime}, 2^{N}\right)}{\bar{C}\left(v, 2^{N}\right)}\right|+\sum_{i=1}^{k}\left|\frac{C_{i}\left(v^{\prime}, 2^{N}\right)}{\bar{C}\left(v, 2^{N}\right)}-\frac{C_{i}\left(v^{\prime}, 2^{N}\right)}{\bar{C}\left(v^{\prime}, 2^{N}\right)}\right|+\varepsilon^{\prime} \\
\leq & \sum_{i=1}^{k}\left|\frac{C_{i}\left(v, 2^{N}\right)-C_{i}\left(v^{\prime}, 2^{N}\right)}{\bar{C}\left(v, 2^{N}\right)}\right|+\left|\frac{\bar{C}\left(v^{\prime}, 2^{N}\right)-\bar{C}\left(v, 2^{N}\right)}{\bar{C}\left(v^{\prime}, 2^{N}\right) \cdot \bar{C}\left(v, 2^{N}\right)}\right| \cdot \sum_{i=1}^{k} C_{i}\left(v^{\prime}, 2^{N}\right)+\varepsilon^{\prime} \\
\leq & \left(k f_{1}(k)+f_{2}(k)+1\right) \cdot \varepsilon^{\prime},
\end{aligned}
$$

where we have used the triangle inequality for absolute values, $\sum_{i=1}^{k} C_{i}\left(v^{\prime}, 2^{N}\right)=\bar{C}\left(v^{\prime}, 2^{N}\right)$, and local approximability.

The requirements of Theorem 4.2 are almost in one-to-one correspondence to those from Theorem 4.1 except the additional requirement $\varepsilon<\frac{1}{k+1}$ for the Alon-Edelman result. This condition is indeed necessary also in our context if we want to prevent from the case that $\Gamma(v)=v^{\prime}$ equals either one of the non-Boolean games $(\emptyset,[n])$ or $\left(2^{[n]},[n]\right)$, see Corollary 4.10 . As it will turn out in Lemma 4.15 we can choose $f_{2}(k)=k+1$ for the absolute Banzhaf index, the following lemma completes the correspondence (except for the tightness of the proposed upper bound).

Lemma 4.3. With the notation from Theorem 4.2 let $\varepsilon<\frac{1}{f_{2}(k)}$ or $\varepsilon^{\prime}<\frac{1}{f_{2}(k)}$. If we additionally assume that $P((\emptyset,[n]))=P\left(\left(2^{[n]},[n]\right)\right)=\mathbf{0}$ and $P(v) \neq \mathbf{0}$, then $v^{\prime}=\Gamma(v) \notin\left\{(\emptyset,[n]),\left(2^{[n]},[n]\right)\right\}$.

Proof. At first we remark that $\sum_{i=k+1}^{n} P_{i}(v) \leq \varepsilon \cdot \sum_{i=1}^{n} P_{i}(v)$ is equivalent to $\sum_{i=k+1}^{n} \widehat{P}_{i}(v) \leq \varepsilon^{\prime}$ for $\varepsilon=\varepsilon^{\prime}$, since $\widehat{P}$ is efficient. Thus we assume $\varepsilon<\frac{1}{f_{2}(k)}$ and it suffices to show $P\left(v^{\prime}\right) \neq \mathbf{0}$. If to the contrary $P\left(v^{\prime}\right)=\mathbf{0}$, then

$$
\|P(v)\|_{1}=\left\|P\left(v^{\prime}\right)-P(v)\right\|_{1}=\left|\sum_{i=1}^{n} P_{i}(v)\right| \leq\left(f_{2}(k)+1\right) \varepsilon \cdot\|P(v)\|_{1}
$$

due to local approximability, the relation $P_{i}(v)=C_{i}\left(v, 2^{N}\right)$, and $\sum_{i=1}^{n} P_{i}(v)=\|P(v)\|_{1}$. For $\varepsilon<\frac{1}{f_{2}(k)}$ and $\|P(v)\|_{1}>0$ this is impossible.

Anticipating Lemma 4.15 we remark that Theorem 4.2 yields a tighter bound for the special case of the Banzhaf index than Theorem 4.1, since we have

$$
\frac{(2 k+1) \varepsilon}{1-(k+1) \varepsilon}+\varepsilon>(2 k+2) \varepsilon
$$


for all $k, \varepsilon>019$,

An application of Theorem 4.2 is given in Subsection 4.2. In the following subsections we give the necessary technical framework and determine for which power indices the stated conditions are satisfied (some cases will remain open nevertheless). For some of the power indices introduced in Section 3 we can easily see that it makes no sense to ask for an Alon-Edelman type result like Theorem 4.2. Our key assumption is that most of the power is concentrated on the first $k$ voters. Some power indices from the literature have the property that players individual power can differ only up to a fix multiplicative constant independently from the given game.

Lemma 4.4. For each simple game $v \in \mathcal{S}_{n}$ we have

$$
\begin{array}{r}
\frac{1}{2} \mathrm{~KB}_{j}(v) \leq \mathrm{KB}_{i}(v) \leq 2 \mathrm{~KB}_{j}(v), \\
\frac{1}{4} \mathrm{PHI}_{j}(v) \leq \mathrm{PHI}_{i}(v) \leq 4 \mathrm{PHI}_{j}(v), \\
\frac{1}{2} \operatorname{Chow}_{j}(v) \leq \operatorname{Chow}_{i}(v) \leq 2 \operatorname{Chow}_{j}(v), \\
\frac{1}{2} \widehat{\mathrm{KB}}_{j}(v) \leq \widehat{\mathrm{KB}}_{i}(v) \leq 2 \widehat{\mathrm{KB}}_{j}(v), \\
\frac{1}{4} \widehat{\mathrm{PHI}}_{j}(v) \leq \widehat{\mathrm{PHI}}_{i}(v) \leq 4 \widehat{\mathrm{PHI}}_{j}(v) \text { and } \\
\frac{1}{2} \widehat{\operatorname{Chow}}_{j}(v) \leq \widehat{\operatorname{Chow}}_{i}(v) \leq 2 \widehat{\mathrm{Chow}}_{j}(v)
\end{array}
$$

for all $i, j \in[n]$.

Proof. Let $v=(\mathcal{W},[n]) \in \mathcal{S}_{n}, i \in[n]$ be arbitrary , and set $A_{i}=\mathcal{W} \backslash \mathcal{W}_{i}$, i.e. the set of winning coalitions that do not contain player $i$. Since $v$ is simple we have $\left|A_{i}\right| \leq\left|\mathcal{W}_{i}\right|$, so that

$$
\frac{1}{2} \cdot|\mathcal{W}| \leq\left|\mathcal{W}_{i}\right| \leq|\mathcal{W}| \leq 2 \cdot\left|\mathcal{W}_{i}\right|
$$

This directly gives the two inequalities for Chow and KB. Estimating the numerator and denominator of PHI separately, gives the stated inequality for the Public Help index. Since the normalization is the same for every player, we can also conclude the three remaining inequalities.

We remark that more explicitly $\mathrm{KB}_{i}(v) \in\left[\frac{1}{2}, 1\right], \widehat{\mathrm{KB}}_{i}(v) \in\left(\frac{1}{2 n}, \frac{2}{n}\right), \mathrm{PHI}_{i}(v) \in\left[\frac{1}{2 n}, \frac{2}{n}\right]$, and $\widehat{\mathrm{PHI}}_{i}(v) \in\left(\frac{1}{4 n}, \frac{4}{n}\right)$ for all simple games $v \in \mathcal{S}_{n}$ and all players $i \in[n]$.

\subsection{Shortenings and locally approximable power indices.}

Definition 4.5. For a Boolean game $v=(\mathcal{W},[n])$ and for $A \subseteq[k]$ we define the reduced game ${ }^{20}$ $v_{A}=\left(\mathcal{W}_{A},(k, n]\right)$, where $\mathcal{W}_{A}=\{B \subseteq(k, n]: A \cup B \in \mathcal{W}\}$.

We remark that $v_{A}$ consists of $n-k$ players. It may happen that $v_{A}$ is not a Boolean game, but it can be easily figured out that there are just three cases:

Lemma 4.6. For a Boolean game $v=(\mathcal{W},[n]) \in \mathcal{B}_{n}$ and a subset $A \subseteq[k]$, where $0<k<n$, (using the notation from Definition 4.5) we have one of the following possibilities:

(1) $\mathcal{W}_{A}=\emptyset$;

(2) $\mathcal{W}_{A}=2^{(k, n]}$;

(3) $v_{A}=\left(\mathcal{W}_{A},(k, n]\right) \in \mathcal{B}_{n-k}$.

We remark that the definition of $k$-pure for a Boolean game $v=(\mathcal{W},[n])$ is equivalent to requiring $\mathcal{W}_{A} \in\left\{\emptyset, 2^{(k, n]}\right\}$ for all $A \subseteq[k]$. If we aim to only perform a relatively small number of modifications to obtain a $k$-pure game $v^{\prime}$ from a given $n$-pure game $v$, it makes sense to modify $\mathcal{W}_{A}$ only if it is not contained in $\left\{\emptyset, 2^{(k, n]}\right\}$. Whenever $\mathcal{W}_{A} \in \mathcal{B}_{n-k}$ we have two choices for the modification.

\footnotetext{
${ }^{19} \mathrm{We}$ remark that the differences in the upper bounds are due to the tighter estimate of $\left|\widehat{\mathrm{Bz}}_{i}\left(v, 2^{N}\right)-\widehat{\mathrm{Bz}}_{i}\left(v^{\prime}, 2^{N}\right)\right|$ in the second part of the proof of Theorem 4.2 compared to the estimation in [1]. The generalized bound would have been $\left\|\widehat{P}\left(v^{\prime}\right)-\widehat{P}(v)\right\|_{1} \leq \frac{\left(2 k f_{1}(k)+1\right) \varepsilon^{\prime}}{1-\left(k f_{1}(k)+1\right) \varepsilon^{\prime}}+\varepsilon^{\prime}$ using the original proof.

${ }^{20}$ See also [77] Definition 1.4.4, 1.4.7].
} 
Definition 4.7. Let $\mathcal{V}_{n}$ be a class of binary games on $n$ players. A mapping $\Gamma: \mathcal{V}_{n} \times[n] \rightarrow \mathcal{V}_{n}$ is a shortening function $\left(\right.$ on $\left.\mathcal{V}_{n}\right)$ if

(1) $v^{\prime}=\Gamma(v, k)$ is $k$-pure for all $k \in[n], v \in \mathcal{V}_{n}$ and

(2) for all $U \subseteq[k]$ with $\mathcal{W}_{U} \in\left\{\emptyset, 2^{(k, n]}\right\}$ we have $v(U \cup V)=v^{\prime}(U \cup V)$ for all $V \in(k, n]$.

One special shortening function, that was already used in [1], is given by some kind of rounding procedure:

Definition 4.8. For a given Boolean game $v=(\mathcal{W},[n])$ and an integer $1 \leq k \leq n$ we denote by $v^{\prime}=(\mathcal{W},[n])$ the game that arises from $v$ as follows: for every $A \subseteq[k]$ we set $\mathcal{W}_{A}^{\prime}=\emptyset$ if $\left|\mathcal{W}_{A}\right| \leq\left|\overline{\mathcal{W}_{A}}\right|$ and $\mathcal{W}_{A}^{\prime}=2^{(k, n]}$ if $\left|\mathcal{W}_{A}\right|>\left|\overline{\mathcal{W}_{A}}\right|$, where $\overline{\mathcal{W}_{A}}=2^{(k, n]}-\mathcal{W}_{A}$ denotes the complement. We call the mapping $\Gamma$ that maps $(v, k)$ to $v^{\prime}$ the $k$-rounding.

The idea behind Definition 4.8 is to obtain a shortening function ${ }^{21}$ that modifies the minimal number of coalitions, i.e. the number of switches from winning to losing, or the other way round, is minimized. We remark that $k$-rounding of a Boolean game $v=(\mathcal{W},[n])$ may result in a game $v^{\prime}=\left(\mathcal{W}^{\prime},[n]\right)$, where $\emptyset \in \mathcal{W}^{\prime}$ or $[n] \notin \mathcal{W}^{\prime}$, i.e. that $v^{\prime}$ is not a Boolean game. But these are the only exceptions and $k$-rounding preserves a number of meaningful properties:

Lemma 4.9. Let $v=(\mathcal{W},[n]) \in \mathcal{B}_{n}$ be a Boolean game and $1 \leq k \leq n$ be an integer such that the $k$-rounding $v^{\prime}=\left(\mathcal{W}^{\prime},[n]\right)$ of $v$ is not equal to either $(\emptyset,[n])$ or $\left(2^{[n]},[n]\right)$, then we have the following implications

(1) $\emptyset \notin \mathcal{W}^{\prime},[n] \in \mathcal{W}^{\prime} \Rightarrow v^{\prime} \in \mathcal{B}_{n}$;

(2) $v$ simple $\Rightarrow v^{\prime}$ simple;

(3) $v$ complete $\Rightarrow v^{\prime}$ complete;

(4) $v$ weighted $\Rightarrow v^{\prime}$ weighted;

(5) $v$ proper $\Rightarrow v^{\prime}$ proper;

(6) $v$ strong and simple $\Rightarrow v^{\prime}$ strong.

Proof.

(1) Obviously $v^{\prime}$ is a function of the form $2^{N} \rightarrow\{0,1\}$ with $N=[n]$. The two other conditions form exactly the missing part of Definition 2.1

(2) Let $S \subseteq T \subseteq[k]$ be two fixed coalitions and $U \subseteq(k, n]$ be arbitrary. If $S \cup U$ is winning in $(\mathcal{W},[n])$, then $T \cup U$ is winning in $(\mathcal{W},[n])$ since $\mathcal{W}$ is simple. Thus we have

$$
|\{U \subseteq(k, n]: S \cup U \in \mathcal{W}\}| \leq|\{U \subseteq(k, n]: T \cup U \in \mathcal{W}\}| .
$$

If $S$ is winning in $\left(\mathcal{W}^{\prime},[n]\right)$, so is $T$. It remains to show $\emptyset \notin \mathcal{W}^{\prime}$ and $[n] \in \mathcal{W}^{\prime}$. Assume $\emptyset \in \mathcal{W}^{\prime}$, then all coalitions $\emptyset \subseteq S \subseteq[n]$ are winning, which contradicts $\mathcal{W}^{\prime} \neq 2^{[n]}$. Similarly assume $[n] \notin \mathcal{W}^{\prime}$, then all coalitions $\emptyset \subseteq S \subseteq[n]$ are losing, which contradicts $\mathcal{W}^{\prime} \neq \emptyset$. Thus $v^{\prime}=\left(\mathcal{W}^{\prime},[n]\right)$ is simple.

(3) Since every complete game is simple we conclude $v^{\prime} \in \mathcal{S}_{n}$ from (2). Now let $S, T \subseteq[k]$ be two fixed coalitions with $S \preceq T$ in $\mathcal{W}$ and $U \subseteq(k, n]$ be arbitrary. We have that $S \cup U \preceq$ $T \cup U$ in $\mathcal{W}$ so that

$$
|\{U \subseteq(k, n]: S \cup U \in \mathcal{W}\}| \leq|\{U \subseteq(k, n]: T \cup U \in \mathcal{W}\}| .
$$

Thus $S \preceq T$ in $v^{\prime}$ and $v^{\prime}=\left(\mathcal{W}^{\prime},[n]\right)$ is complete.

(4) Since every weighted game is simple, we conclude $v^{\prime} \in \mathcal{S}_{n}$ from (2). Now let $\left[q ; w_{1}, \ldots, w_{n}\right]$ be a weighted representation of $v$, where we w.l.o.g. assume $w_{i} \geq 0$ for all $1 \leq i \leq n$. Next we use the weights $w_{1}^{\prime}=w_{1}, \ldots, w_{k}^{\prime}=w_{k}, w_{k+1}^{\prime}=0, \ldots, w_{n}^{\prime}=0$, i.e. $w^{\prime}(X)=w^{\prime}(X \cap$ $[k])$ for all coalitions $X \subseteq[n]$. With this we denote the minimum weight $w^{\prime}(X)$ of a winning coalition in $X \in \mathcal{W}^{\prime}$ by $u$ and the maximum weight $w^{\prime}(X)$ of a losing coalition $X \in \mathcal{W}^{\prime}$ by $l$. Let $S \subseteq[1, k]$ be a winning coalition $S \in \mathcal{W}^{\prime}$ with weight $w^{\prime}(S)=u$ and $T \subseteq[1, k]$ be a losing coalition $T \notin \mathcal{W}^{\prime}$ with weight $w^{\prime}(T)=l$. For an arbitrary coalition $V \subseteq[1, k]$,

\footnotetext{
${ }^{21}$ The operation $k$-rounding is indeed a shortening function $\mathcal{V}_{n} \times[n] \rightarrow \mathcal{V}_{n}$ for many of the most meaningful of classes $\mathcal{V}_{n}$ of binary games mildly modified to satisfy a technical condition, see Lemma 4.9 and Corollary 4.10
} 
with $w^{\prime}(V) \geq w^{\prime}(S)=u$, we have $w(V) \geq w(S)=u$ and $w(V \cup U) \geq w(S \cup U)$ for all $U \subseteq(k, n]$, so that

$$
|\{U \subseteq(k, n]: V \cup U \in \mathcal{W}\}| \geq|\{U \subseteq(k, n]: S \cup U \in \mathcal{W}\}| .
$$

Thus, $V$ has to be winning in $v^{\prime}=\left(\mathcal{W}^{\prime},[n]\right)$ and we clearly have $l<u$.

For an arbitrary coalition $V^{\prime} \subseteq[1, k]$ with $w^{\prime}\left(V^{\prime}\right)<w^{\prime}(S)=u$, we have $w\left(V^{\prime}\right)<$ $w(S)=u$ and coalition $V^{\prime}$ is losing in $v^{\prime}$ due to the definition of $u$. Thus, $w^{\prime}\left(V^{\prime}\right) \leq w^{\prime}(T)=$ $l$ due to the definition of $l$ and $\left[q^{\prime} ; w_{1}, \ldots, w_{k}, 0, \ldots, 0\right]$ is a weighted representation of $v^{\prime}$ for each $q^{\prime} \in(l, u]$.

(5) Let $S \subseteq[1, k]$ be an arbitrary coalition and $T=[1, k] \backslash S$. By $m_{1}$ we denote the number of coalitions $U \subseteq(k, n]$ such that $S \cup U$ is winning in $v$. Similarly, by $m_{2}$ we denote the number of coalitions $U \subseteq(k, n]$ such that $T \cup((k, n] \backslash U)$ is losing in $v$. If $S \cup U$ is winning in $v$, where $U \subseteq(k, n]$, then $T \cup((k, n] \backslash U)$ is losing in $v$ due to the fact that $v$ is proper. Thus, we have $m_{1}+m_{2} \leq 2^{n-k}$. Due to the rounding procedure, $S$ is winning in $v^{\prime}$ iff $m_{1}>2^{n-k-1}$. Similarly, $T$ is winning in $v^{\prime}$ iff $m_{2}>2^{n-k-1}$. Both cases cannot occur simultaneously so that we conclude that $v^{\prime}$ is proper.

(6) We use the same notation as in (5). If $S \cup U$ is losing in $v$, where $U \subseteq(k, n]$, then $T \cup$ $((k, n] \backslash U)$ is winning in $v$ due to the fact that $v$ is strong. Thus $m_{1}+m_{2} \geq 2^{n-k}$. Due to the rounding procedure $S$ is losing in $v^{\prime}$ iff $m_{1} \leq 2^{n-k-1}$ and $T$ is losing in $v^{\prime}$ iff $m_{2} \leq 2^{n-k-1}$. Thus both coalitions can be losing simultaneously in $v^{\prime}$ if and only if $m_{1}=m_{2}=2^{n-k-1}$, i.e. exactly one of the two coalitions $S \cup U$ and $T \cup((k, n] \backslash U)$ is winning in $v$ for all $U \subseteq(k, n]$. Since game is strong, $m_{1}>0$, and $v$ is simple the coalition $S \cup(k, n]$ has to be winning in $v$. Thus $T \cup \emptyset$ has to be losing in $v$. But then all coalitions $T \cup((k, n] \backslash U)$ would be losing so that $m_{2}=0$. Thus the case $m_{1}=m_{2}=2^{n-k-1}$ is not possible for simple games and we can conclude that $v^{\prime}$ is strong.

For $\left|\mathcal{W}_{A}\right|=\left|\overline{\mathcal{W}_{A}}\right|$ the chosen tie-breaking rule in Definition 4.8 makes the additional assumption of being a simple game in implication (6) of Lemma 4.9 necessary. If we modify the tie-breaking rule in the other direction implications (1)-(4) and implication (6), without the assumption of being a simple game, remain valid. For implication (5) we then need the assumption of being a simple game (or possibly a relaxation).

Corollary 4.10. For each integer $1 \leq k<n$ the operation of $k$-rounding, interpreted as a mapping $\mathcal{V}_{n} \times[n] \rightarrow \mathcal{V}_{n}$, is a shortening function for

$$
\mathcal{V}_{n} \in\left\{\left\{v: v: 2^{[n]} \rightarrow\{0,1\}\right\}, \mathcal{S}_{n} \cup \mathcal{X}_{n}, \mathcal{C}_{n} \cup \mathcal{X}_{n}, \mathcal{T}_{n} \cup \mathcal{X}_{n}\right\}
$$

where $\mathcal{X}_{n}=\left\{(\emptyset,[n]),\left(2^{[n]},[n]\right)\right\}$.

Definition 4.11. Let $\mathcal{V}_{n}$ be a class of binary games on $n$ players, $\Gamma: \mathcal{V}_{n} \times[n] \rightarrow \mathcal{V}_{n}$ be a shortening function on $\mathcal{V}_{n}$, and $P: \mathcal{V}_{n} \rightarrow \mathbb{R}_{\geq 0}^{n}$ be a power index induced by a counting function $C: \mathcal{V}_{n} \times$ $2^{[n]} \times[n] \rightarrow \mathbb{R}_{\geq 0}^{n}$ on $\mathcal{V}_{n}$. We say that $P$ and $C$ are locally approximable for $\Gamma$ (on $\mathcal{V}_{n}$ ), if there exist functions $f_{1}: \overline{\mathbb{N}}_{>0} \rightarrow \mathbb{R}_{\geq 0}$ and $f_{2}: \mathbb{N}_{>0} \rightarrow \mathbb{R}_{\geq 0}$ such that for all integers $1 \leq k<n$, all real constants $\varepsilon \geq 0$, and all games $v=(\mathcal{W},[n]) \in \mathcal{V}_{n}$ with $\sum_{i=k+1}^{n} C_{i}\left(v, 2^{N}\right) \leq \varepsilon \cdot \bar{C}\left(v, 2^{N}\right)$, we have

(1) $C_{i}\left(v, 2^{N}\right)-f_{1}(k) \cdot \varepsilon \cdot \bar{C}\left(v, 2^{N}\right) \leq C_{i}\left(\Gamma(v), 2^{N}\right) \leq C_{i}\left(v, 2^{N}\right)+f_{1}(k) \cdot \varepsilon \cdot \bar{C}\left(v, 2^{N}\right)$

(2) $\bar{C}\left(v, 2^{N}\right)-f_{2}(k) \cdot \varepsilon \cdot \bar{C}\left(v, 2^{N}\right) \leq \bar{C}\left(\Gamma(v), 2^{N}\right) \leq \bar{C}\left(v, 2^{N}\right)+f_{2}(k) \cdot \varepsilon \cdot \bar{C}\left(v, 2^{N}\right)$

for all $1 \leq i \leq k$, where $N=[n]$.

In other words, the assumptions from Definition 4.11 say that, if for a given game $v$ the contribution $\sum_{i=k+1}^{n} C_{i}\left(v, 2^{N}\right)$ of the last $n-k$ players is relatively small, compared to the total contribution $\bar{C}\left(v, 2^{N}\right)$, then the aggregated counting function $C_{i}\left(\Gamma(v), 2^{N}\right)$ of the shortened game $\Gamma(v)$ is near ${ }^{22}$ to the aggregated counting function $C_{i}\left(v, 2^{N}\right)$ of the game itself.

If a given locally approximable counting function is multiplied by a positive constant factor, not depending on the respective game, then the quality functions remain valid:

\footnotetext{
22 with respect to the $\|\cdot\|_{1}$-norm
} 
Lemma 4.12. Let $\mathcal{V}_{n}$ be a class of binary games on $n$ players, $\Gamma: \mathcal{V}_{n} \times[n] \rightarrow \mathcal{V}_{n}$ be a shortening function on $\mathcal{V}_{n}$, and $P: \mathcal{V}_{n} \rightarrow \mathbb{R}_{\geq 0}^{n}$ be a power index induced by a counting function $C: \mathcal{V}_{n} \times 2^{[n]} \times$ $[n] \rightarrow \mathbb{R}_{\geq 0}^{n}$ on $\mathcal{V}_{n}$. If $P$ and $C$ are locally approximable for $\Gamma$ on $\mathcal{V}_{n}$ with quality functions $f_{1}$ and $f_{2}$, then $C^{\prime}=C \cdot \Lambda$ and the corresponding induced power index $P^{\prime}=P \cdot \Lambda$ are locally approximable for $\Gamma$ on $\mathcal{V}_{n}$ with the same quality functions $f_{1}$ and $f_{2}$, where $\Lambda \in \mathbb{R}_{>0}$.

We remark that there is a canonical choice for the function $f_{2}$ when we are only given a function $f_{1}$ satisfying the respective requirements from Definition 4.11

Lemma 4.13. If $f_{1}$ satisfies condition (1) of Definition 4.11 then $f_{2}(k):=k \cdot f_{1}(k)+1$ satisfies condition (2) of Definition 4.11

Corollary 4.14. Given the assumptions of Theorem 4.2 with $f_{2}(k):=k \cdot f_{1}(k)+1$ according to Lemma 4.13 we have

$$
\left\|\widehat{P}\left(v^{\prime}\right)-\widehat{P}(v)\right\|_{1} \leq\left(2 k f_{1}(k)+2\right) \varepsilon^{\prime}
$$

For many power indices we will subsequently only state a suitable choice for $f_{1}$ and not for $f_{2}$, whenever we do not know a feasible function $f_{2}$ such that the corresponding bounds of Theorem 4.2 are tighter than those from Corollary 4.14. For some power indices a tighter choice for $f_{2}$ pays off.

Lemma 4.15. The counting function $C^{\mathrm{Bz}}$ of the absolute Banzhaf index is locally approximable, with $f_{1}^{\mathrm{Bz}}(k)=1$ and $f_{2}^{\mathrm{Bz}}(k)=k+1$, for $k$-rounding on all binary voting classes $\mathcal{V}_{n}$, where $k$-rounding is a shortening function, i.e. where games remain in $\mathcal{V}_{n}$ after rounding.

To ease notation we will, instead of this precise technical statement, say that $f_{1}^{\mathrm{Bz}}(k)=1$ and $f_{2}^{\mathrm{Bz}}(k)=k+1$ are feasible for $C^{\mathrm{Bz}}$ and $k$-rounding in the following and in similar situations. As in Theorem 4.2 we call $f_{1}, f_{2}$ quality functions.

Proof. (of Lemma 4.15, compare [1])

Let $v=(\mathcal{W},[n])$ be a game. Suppose that for $i \in(k, n]$ and $B \subseteq[n]$ we have that $i \notin B, B \notin \mathcal{W}$, but $B \cup i \in \mathcal{W}$. Let $A=B \cap[1, k]$ and $B^{\prime}=B-A$, then $i \notin B^{\prime}, B^{\prime} \notin \mathcal{W}_{A}$, but $B^{\prime} \cup i \in \mathcal{W}_{A}$. This correspondence implies that for every $k+1 \leq j \leq n$ we have

$$
C_{j}^{\mathrm{Bz}}\left(v, 2^{N}\right)=\sum_{A \subseteq[k]} C_{j}^{\mathrm{Bz}}\left(\left(\mathcal{W}_{A},(k, n]\right), 2^{N}\right) .
$$

From the edge-isoperimetric inequality for the cube-graph ${ }^{23}$ one concludes

$$
\bar{C}^{\mathrm{Bz}}\left((\mathcal{F},[n]), 2^{N}\right) \geq|\mathcal{F}|\left(n-\log _{2}|\mathcal{F}|\right)
$$

for all $\mathcal{F} \subseteq 2^{[n]}$, so that

$$
\bar{C}^{\mathrm{Bz}}\left((\mathcal{F},[n]), 2^{N}\right) \geq \min \{|\mathcal{F}|,|\overline{\mathcal{F}}| \mid\}
$$

Thus we have

$$
\begin{aligned}
\varepsilon \cdot \bar{C}^{\mathrm{Bz}}\left(v, 2^{N}\right) & \geq \sum_{i=k+1}^{n} C_{i}^{\mathrm{Bz}}\left(v, 2^{N}\right)=\sum_{i=k+1}^{n} \sum_{A \subseteq[k]} C_{i}^{\mathrm{Bz}}\left(\left(\mathcal{W}_{A},(k, n]\right), 2^{N}\right) \\
& \geq \sum_{A \subseteq[k]} \min \left\{\left|\mathcal{W}_{A}\right|,\left|\overline{\mathcal{W}_{A}}\right|\right\} .
\end{aligned}
$$

From Lemma 4.16, see below, we then conclude the feasibility of $f_{1}^{\mathrm{Bz}}(k)=1$, so that Lemma 4.13 then gives the feasibility of $f_{2}^{\mathrm{Bz}}(k)=k f_{1}^{\mathrm{Bz}}(k)+1=k+1$.

Lemma 4.16. [29, Lemma 3.3.12] Let $v=(\mathcal{W}, N)$ be a simple game and $N \neq T \in \mathcal{W}$ be a minimal winning coalition. For the simple game $v^{\prime}$ arising by deleting $T$ from the set of winning coalitions we have

$$
\eta_{i}\left(v^{\prime}\right)= \begin{cases}\eta_{i}(v)-1 & : \quad i \in T \\ \eta_{i}(v)+1 & : \quad i \in N \backslash T .\end{cases}
$$

\footnotetext{
${ }^{23}$ See also [26] Corollary 1].
} 
We remark that for all of the power indices introduced in Section 3, except for the nucleolus and the MSR index, similar recursion formulas as in Lemma 4.16 exist.

From Lemma 4.12 and Lemma4.15 we conclude:

Corollary 4.17. The quality functions $f_{1}(k)=1$ and $f_{2}(k)=k+1$ are feasible for the vector of the number of $i$-swings $\left(\eta_{1}, \ldots, \eta_{n}\right)$, i.e. the Banzhaf score, given by the counting function

$$
C_{i}(v, S)= \begin{cases}1 & : \quad i \in S, v(S)=1, v(S-i)=0, \\ 0 & : \text { otherwise }\end{cases}
$$

and k-rounding.

Lemma 4.18. Let the quality functions $f_{1}$ and $f_{2}$ be feasible for a power index $P$ induced by $a$ counting function $C$ and $\alpha \in \mathbb{R}_{\geq 0}$ be a constant. The shifted counting function $C_{i}^{\prime}(v, S)=C_{i}(v, S)+$ $\frac{\alpha}{2^{n}}$, where $n$ is the number of players in $v$, induces the power index $P^{\prime}=P+\alpha$ and $f_{1}, f_{2}$ are feasible for $C^{\prime}$ and $P^{\prime}$ too.

Proof. At first we observe

$$
P_{i}^{\prime}(v)=\sum_{S \subseteq[n]} C_{i}^{\prime}(v, S)=\alpha+\sum_{S \subseteq[n]} C_{i}(v, S)=\alpha+P_{i}(v) .
$$

With the notation of Definition 4.11 we have

$$
\begin{aligned}
C_{i}^{\prime}\left(\Gamma(v), 2^{N}\right) & =C_{i}\left(\Gamma(v), 2^{N}\right)+\alpha \\
& \leq C_{i}\left(v, 2^{N}\right)+\alpha+f_{1}(k) \cdot \varepsilon \cdot \bar{C}\left(v, 2^{N}\right) \\
& =C_{i}^{\prime}\left(v, 2^{N}\right)+f_{1}(k) \cdot \varepsilon \cdot \bar{C}\left(v, 2^{N}\right) \\
& \leq C_{i}^{\prime}\left(v, 2^{N}\right)+f_{1}(k) \cdot \varepsilon \cdot \bar{C}^{\prime}\left(v, 2^{N}\right),
\end{aligned}
$$

since $f_{1}(k), \varepsilon, \alpha \geq 0$. Similarly we obtain

$$
C_{i}^{\prime}\left(\Gamma(v), 2^{N}\right) \geq C_{i}^{\prime}\left(v, 2^{N}\right)-f_{1}(k) \cdot \varepsilon \cdot \bar{C}^{\prime}\left(v, 2^{N}\right)
$$

and

$$
\bar{C}^{\prime}\left(v, 2^{N}\right)-f_{2}(k) \cdot \varepsilon \cdot \bar{C}^{\prime}\left(v, 2^{N}\right) \leq \bar{C}^{\prime}\left(\Gamma(v), 2^{N}\right) \leq \bar{C}^{\prime}\left(v, 2^{N}\right)+f_{2}(k) \cdot \varepsilon \cdot \bar{C}^{\prime}\left(v, 2^{N}\right) .
$$

In combination Lemma 4.12 and Lemma 4.18 say that we can transfer quality functions for a given power index $P$, induced by counting function, to all power indices $P^{\prime}=\alpha+\beta P$, where $\alpha \in \mathbb{R}_{>0}$ and $\beta \in \mathbb{R}_{>0}$. As an application we mention the following corollary based on the identity $\mathrm{Rae}=\frac{1}{2}+\frac{1}{2} \cdot \mathrm{Bz}$ :

Corollary 4.19. The quality functions $f_{1}(k)=1$ and $f_{2}(k)=k+1$ are feasible for the Rae index and $k$-rounding.

In Subsection 4.3 we will state the respective quality functions, so that Theorem 4.2 can be applied, for most of the power indices introduced in Section 3. Most of the proofs, based on elementary counting and bookkeeping considerations, will be delayed to the appendix. For $p$-binomial semivalues it will turn out that no such result based on $k$-rounding can exist, for the Johnston index even the larger class of shortening functions admits no such result. In order to prove those negative results, we introduce a parameterized class of weighted games in Subsection 4.4. Now we proceed with an application of Theorem 4.2 in the next subsection.

4.2. Applications. We would like to demonstrate how to apply Theorem 4.2 to deduce some information on power vectors that are hard to approximate. Assume that we want to find a simple game with normalized Banzhaf vector $\sigma_{n}=\left(\frac{3}{4}, \frac{1}{4}, \ldots\right)$, where the number of players $n \geq 2$ is arbitrary. It will turn out that no simple game can exactly meet the desired power distribution $\sigma_{n}$, so that we aim at minimizing the $\|\cdot\|_{1}$-distance between $\sigma_{n}$ and the realized Banzhaf distribution $\mathrm{Bz}(v)$. The idea is to use negative approximation results for games on $k<n$ players and then to apply Theorem 4.2 to conclude lower bounds for arbitrary $n$. For this purpose we need to bridge the gap between a $k$ pure game on $n$ players (as in Theorem 4.2) and the resulting $k$-player game after removing the null players. 
Definition 4.20. A power index $P: \mathcal{V}_{i} \rightarrow \mathbb{R}_{>0}^{i}$ is called null player removable if for all $v \in \mathcal{V}_{n}$ and $v^{\prime} \in \mathcal{V}_{k}$, arising from $v$ by deleting the null players, we have $P_{j}(v)=P_{j}\left(v^{\prime}\right)$ for all players $j$, which are not null players in $v$.

Both, the normalized and the absolute Banzhaf index are null player removable while the index based on the number of $i$-swings, is not. For the later case the number of $i$-swings for all non-null players doubles for each added extra null player.

theorem 4.21. Let $0<k<n$ be integers, $\sigma \in \mathbb{R}_{>0}^{n}$ be a desired power vector with $\|\sigma\|_{1}=1, P$ be a power index induced by a counting function $C$ that is locally approximable for the shortening function $\Gamma: \mathcal{V}_{n} \rightarrow \mathcal{V}_{n}$ with quality functions $f_{1}$ and $f_{2}$. If $P$ further is positive, satisfies the null player property, $\widehat{P}$ is null player removable, and $\min \left\{\frac{\Lambda+\alpha}{\beta+2}, \frac{1}{f_{2}(k)}\right\} \geq \alpha$, then we have

$$
\|\widehat{P}(v)-\sigma\|_{1} \geq \min \left\{2 \cdot\left(\frac{1}{f_{2}(k)}-\alpha\right), \frac{2}{\beta+2} \cdot \Lambda-\frac{2 \beta+2}{\beta+2} \cdot \alpha\right\},
$$

where $\sigma^{\prime}=\left(\sigma_{1}, \ldots, \sigma_{k}\right), \alpha=1-\left\|\sigma^{\prime}\right\|_{1}, \beta=f_{2}(k)+k f_{1}(k)+1$, and $\Lambda=\min _{v^{\prime} \in \mathcal{V}_{k}}\left\|\sigma^{\prime}-\widehat{P}\left(v^{\prime}\right)\right\|_{1}$, for all $v \in \mathcal{V}_{n}$.

Proof. Let $v \in \mathcal{V}_{n}$ be arbitrary and $v^{\prime}=\Gamma(v) \in \mathcal{V}_{k}$. For $\varepsilon<\frac{1}{f_{2}(k)}$ with $\sum_{i=k+1}^{i} \widehat{P}_{i}(v) \leq \varepsilon$ we obtain

$$
\left\|\widehat{P}\left(v^{\prime}\right)-\widehat{P}(v)\right\|_{1} \leq\left(f_{2}(k)+k f_{1}(k)+1\right) \varepsilon
$$

from Theorem 4.2. Applying the triangle inequality for $\|\cdot\|_{1}$ gives

$$
\|\widehat{P}(v)-\sigma\|_{1} \geq\left\|\widehat{P}\left(v^{\prime}\right)-\sigma^{\prime}\right\|_{1}-\left\|\widehat{P}\left(v^{\prime}\right)-\widehat{P}(v)\right\|_{1}-\left(1-\left\|\sigma^{\prime}\right\|_{1}\right) .
$$

If otherwise $\sum_{i=k+1}^{i} \widehat{P}_{i}(v)>\varepsilon$, then

$$
\|\widehat{P}(v)-\sigma\|_{1}=\sum_{i=1}^{k}\left|\widehat{P}_{i}(v)-\sigma_{i}\right|+\sum_{i=k+1}^{n}\left|\widehat{P}_{i}(v)-\sigma_{i}\right| \geq 2 \cdot \max \left\{\left\|\sigma^{\prime}\right\|_{1}-1+\varepsilon, 0\right\} .
$$

Now it remains to choose a suitable $\varepsilon$ such that the weakest of the two lower bounds is maximized. If $\frac{\Lambda+\alpha}{\beta+2}<\frac{1}{f_{2}(k)}$, then we can choose $\varepsilon=\frac{\Lambda+\alpha}{\beta+2}$ so that both right hand sides are equal to $\frac{2}{\beta+2} \cdot \Lambda-\frac{2 \beta+2}{\beta+2} \cdot \alpha$. Now assume $\frac{\Lambda+\alpha}{\beta+2} \geq \frac{1}{f_{2}(k)}$. If $\alpha=\frac{1}{f_{2}(k)}$, then the proposed inequality is true due to $\|\widehat{P}(v)-\sigma\|_{1} \geq 0$, so that we can additionally assume $\frac{1}{f_{2}(k)}>\alpha$. Finally we consider

$$
\lim _{\varepsilon \rightarrow \frac{1}{f_{2}(k)}: \alpha<\varepsilon<\frac{1}{f_{2}(k)}} \min \{\Lambda-\alpha-\beta \epsilon, 2(\varepsilon-\alpha)\}=2 \cdot\left(\frac{1}{f_{2}(k)}-\alpha\right) .
$$

As an example we choose $n \geq 3, \sigma=\left(\frac{29}{40}, \frac{9}{40}, \frac{1}{20(n-2)}, \ldots, \frac{1}{20(n-2)}\right), P=\mathrm{Bz}, \Gamma$ as $k$-rounding and $\mathcal{V}_{i}=\mathcal{S}_{i} \cup\left\{(\emptyset,[i]),\left(2^{[i]},[i]\right)\right\}$, i.e. the set of simple games supplemented by the two trivial nonBoolean games for technical reasons. For $k=2$ we have $\alpha=\frac{1}{20}$ and $\Lambda=\frac{1}{2}$, since the only achievable Banzhaf distributions are $(1,0),\left(\frac{1}{2}, \frac{1}{2}\right)$, and $(0,1)$. From Lemma 4.15 we read of $\beta=6$ and $f_{2}(2)=3$. Inserting yields $\|\widehat{\mathrm{Bz}}(v)-\sigma\|_{1} \geq \frac{3}{80}$ for all $v \in \mathcal{S}_{n}$, i.e. $\sigma$ cannot be approximated too well by Banzhaf distributions within the class of simple games.

Corollary 4.22. Let $1<k<n$ be integers, $\sigma=\left(\sigma_{1}, \ldots, \sigma_{k}, 0, \ldots, 0\right) \in \mathbb{R}_{\geq 0}^{n}$ be a desired power vector with $\|\sigma\|_{1}=1, P \in\{\mathrm{Bz}, \mathrm{ColPrev}$, ColIni, PGI, DP, Shift, SDP $\}$ be a power index, $\mathcal{V} \in$ $\{\mathcal{S}, \mathcal{C}, \mathcal{T}\}$ be a class of games, then

$$
\|\widehat{P}(v)-\sigma\|_{1} \geq \min \left\{\frac{2}{f_{2}^{P}(k)}, \frac{2 \Lambda}{f_{2}^{P}(k)+k f_{1}^{P}(k)+3}\right\},
$$

$\sigma^{\prime}=\left(\sigma_{1}, \ldots, \sigma_{k}\right), \Lambda=\min _{v^{\prime} \in \mathcal{V}_{k}}\left\|\sigma^{\prime}-\widehat{P}\left(v^{\prime}\right)\right\|_{1}$ and the corresponding $f_{1}^{P}, f_{2}^{P}$ can be found in Table 1 for all $v \in \mathcal{V}_{n}$. 
Proof. For $\mathcal{V}_{i}^{\prime}=\mathcal{V}_{i} \cup\left\{(\emptyset,[i]),\left(2^{[i]},[i]\right)\right\}$ the operation $k$-rounding is a shortening function $\mathcal{V}_{n}^{\prime} \rightarrow \mathcal{V}_{i}^{\prime}$. For $v \in\left\{(\emptyset,[n]),\left(2^{[n]},[n]\right)\right\}$ we have $\|\widehat{P}(v)-\sigma\|_{1}=1$ so that the proposed bound is valid due to $f_{2}^{P}(2) \geq 2$ for all cases of $P$. Thus we can assume $v \in \mathcal{V}_{n}$. With the notation from Theorem 4.21 we have $\alpha=0$ and all requirements on $P$ are satisfied. Since $\min \left\{\frac{\Lambda+\alpha}{\beta+2}, \frac{1}{f_{2}(k)}\right\} \geq 0$ and $\min _{v^{\prime} \in \mathcal{V}_{k}}\left\|\sigma^{\prime}-\widehat{P}\left(v^{\prime}\right)\right\|_{1} \geq \min _{v^{\prime} \in \mathcal{V}_{k}^{\prime}}\left\|\sigma^{\prime}-\widehat{P}\left(v^{\prime}\right)\right\|_{1}$ we can conclude the proposed bound from the bound of Theorem 4.21 ,

As an example we consider $\sigma_{n}=\left(\frac{3}{4}, \frac{1}{4}, 0, \ldots, 0\right)$ for $n \geq 3, P=\mathrm{Bz}$, and $k=2$. Corollary 4.22 then yields $\|\widehat{\mathrm{Bz}}(v)-\sigma\|_{1} \geq \frac{1}{8}$ for all simple games $v \in \mathcal{S}_{n}$. We remark that in [46] the bound $\|\widehat{\mathrm{Bz}}(v)-\sigma\|_{1} \geq \frac{1}{9}$ was proven based on the original Alon-Edelman bound. Computational results for the corresponding exact solutions of the inverse power index problem for simple games with up to 11 players lead to the conjecture $\left\|\mathrm{Bz}(v)-\sigma_{n}\right\|_{1} \geq \frac{1}{3}$ (and indeed a even tighter one).

4.3. Quality functions for several power indices. Having the theoretical framework of the preceding subsections at hand it remains to prove the validity of suitable quality functions for the different power indices in order to conclude the respective Alon-Edelman type results. In contrast to the proof of Lemma 4.15 for the Banzhaf index, where the edge-isoperimetric inequality for the cube-graph was used, we will only need elementary counting and bookkeeping arguments. Exemplarily, we state the proof for the quality functions for the Public Good Index. In the following we will abbreviate the term minimal winning coalition by MWC.

Lemma 4.23. For the Public Good Index we can choose $f_{1}(k)=k+1$ and $f_{2}(k)=\frac{(k+1)(k+5)}{4}$.

Proof. Let $v=(\mathcal{W},[n])$ be an arbitrary simple game. As defined before, $C_{i}^{\mathrm{PGI}}\left(v, 2^{[n]}\right)$ counts the number of minimal winning coalitions in $v$ containing player $i$ and we have $\bar{C}^{\mathrm{PGI}}\left(v, 2^{[n]}\right)=$ $\sum_{i=1}^{n} C_{i}^{\mathrm{PGI}}\left(v, 2^{[n]}\right)$. For brevity we just write $C_{i}$ and $\bar{C}$. As further abbreviations we use $v^{\prime}=$ $\left(\mathcal{W}^{\prime},[n]\right)$ for the $k$-rounding of $v, \widehat{C}=\sum_{i=k+1}^{n} C_{i}$, i.e. the restriction of $\bar{C}$ to the players in $(k, n]$, and by $\widehat{M}$ we denote the number of minimal winning coalitions in $\mathcal{W}$ that contain at least one player from $(k, n]$. With this we have $\widehat{M} \leq \widehat{C}$.

Now we want to study the changes in the number $C_{i}$ of minimal winning coalitions by going from $v$ to $v^{\prime}$. At first we consider the cases where a coalition $S$ is a MWC in $v$ but not in $v^{\prime}$.

(1) If $S \cap(k, n] \neq \emptyset$, then $S$ is a MWC containing a player from $(k, n]$, so that for a given index $1 \leq i \leq k, C_{i}$ decreases by at most $\widehat{M}$.

(2) Let us assume $S \subseteq[k]$. Since $S$ is a MWC in $v$, it is indeed winning in $v$. Thus $S \cup T$ is winning in $v$ for all $T \subseteq(k, n]$, so that $S$ is also winning in $v^{\prime}$. Since $S$ is not a MWC in $v^{\prime}$, there exists a player $j \in S$ such that $S-j$ is also winning in $v^{\prime}$. This can only happen if there is a subset $\emptyset \neq T \subseteq(k, n]$ such that $S-j+T$ is a MWC in $v$. So the change of a MWC $S-j+T$ can cause the change of a MWC $S$. Now we want to count how often this can happen. To this end we set $r:=|S-j|$ and remark $1 \leq r \leq k-1$, since $\emptyset$ can not be winning in $v^{\prime}$ (our conditions ensure that also $v^{\prime}$ is a simple game). If $i \in S-j$, then we have $k-r$ possibilities and one possibility otherwise. Thus, for a given index $1 \leq i \leq k, C_{i}$ decreases by at most $\widehat{M}(k-1)$.

The overall decrease of all $C_{i}$ with $1 \leq i \leq k$ can be bounded a bit tighter than $\widehat{M}(k-1) k$ as follows. For $S$ we have $k-r$ possibilities for the $r$ players in $S-j$ and 1 possibility for the remaining $k-r$ players in $[k]$. Since $(r+1)(k-r) \leq \frac{(k+1)^{2}}{4}$, the overall decrease is at $\operatorname{most} \widehat{M} \cdot \frac{(k+1)^{2}}{4}$.

Next we consider the cases where a coalition $S$ is not a MWC in $v$ but a MWC in $v^{\prime}$. Since the players in $(k, n]$ are null players in $v^{\prime}$, we deduce $S \subseteq[k]$.

(3) Assume that $S$ is losing in $v$ but winning in $v^{\prime}$. According to the rounding procedure, there exists a MWC $S \cup T$ in $v$, where $T \subseteq(k, n]$. Thus, for a given index $1 \leq i \leq k, C_{i}$ increases by at most $\widehat{M}$.

(4) If $S$ is winning in $v$, then there must be a player $j \in S$ so that $S-j$ is also winning in $v$, but losing in $v^{\prime}$. According to the rounding procedure, there exists a MWC $S-j \cup T$ in $v$, where 
$T \subseteq(k, n]$. We proceed similarly as in case (2) and set $r:=|S-j|$, so that $0 \leq r \leq k-1$. Thus, for a given index $1 \leq i \leq k, C_{i}$ increases by at most $\widehat{M} \cdot k$ and the overall increase is at most $\widehat{M} \cdot \frac{(k+1)^{2}}{4}$.

Summarizing the four cases gives

$$
C_{i}^{\mathrm{PGI}}\left(v, 2^{N}\right)-(k+1) \widehat{C} \leq C_{i}^{\mathrm{PGI}}\left(v, 2^{N}\right)-k \cdot \widehat{M} \leq C_{i}^{\mathrm{PGI}}\left(v^{\prime}, 2^{N}\right)
$$

and

$$
C_{i}^{\mathrm{PGI}}\left(v^{\prime}, 2^{N}\right) \leq C_{i}^{\mathrm{PGI}}\left(v, 2^{N}\right)+(k+1) \widehat{M} \leq C_{i}^{\mathrm{PGI}}\left(v, 2^{N}\right)+(k+1) \widehat{C}
$$

for all $1 \leq i \leq k$. Since $\widehat{C} \leq \varepsilon \cdot \bar{C}^{\mathrm{PGI}}\left(v, 2^{N}\right)$, we can choose $f_{1}(k)=k+1$.

Using $\widehat{C} \leq \varepsilon \cdot \bar{C}^{\mathrm{PGI}}\left(v, 2^{N}\right)$ and $\sum_{i=k+1}^{n} C_{i}^{\mathrm{PGI}}\left(v^{\prime}, 2^{n}\right)=0$, we obtain

$$
\bar{C}^{\mathrm{PGI}}\left(v, 2^{N}\right)-\frac{(k+1)(k+5)-4}{4} \cdot \widehat{M} \leq \bar{C}^{\mathrm{PGI}}\left(v^{\prime}, 2^{N}\right)
$$

and

$$
\bar{C}^{\mathrm{PGI}}\left(v^{\prime}, 2^{N}\right) \leq \bar{C}^{\mathrm{PGI}}\left(v, 2^{N}\right)+\frac{(k+1)(k+5)-4}{4} \cdot \widehat{M}+\varepsilon \cdot \bar{C}^{\mathrm{PGI}}\left(v, 2^{N}\right),
$$

so that we can choose $f_{2}(k)=\frac{(k+1)(k+5)}{4}$.

In Table 1 we state suitable quality functions for 11 different power indices, whose proofs all are rather similar to that of Lemma 4.23 and therefore shifted to Appendix A. We have to remark, that for the Tijs index we do not use $k$-rounding but $k$-up-rounding, i.e. we set $\mathcal{W}_{A}^{\prime}=2^{(k, n]}$ if $\left|\mathcal{W}_{A}\right| \geq 1$ and $\mathcal{W}_{A}^{\prime}=\emptyset$ (using the notation from Definition 4.8).

\begin{tabular}{rrrr|}
\hline power index & $\mathbf{f}_{\mathbf{1}}(\mathbf{k})$ & $\mathbf{f}_{\mathbf{2}}(\mathbf{k})$ & justification \\
Tijs & 0 & 1 & Lemma A.8 \\
Bz & 1 & $k+1$ & Lemma 4.15 \\
swing $=2^{n-1} \cdot \mathrm{Bz}$ & 1 & $k+1$ & Lemma \\
$\Psi^{P}$ with $c_{1} \leq P_{i} \leq c_{2}$, & & & \\
where $c_{1}, c_{2} \in \mathbb{R}_{>0}, 0 \leq i \leq n-1$ & $\frac{c_{2}}{c_{1}}$ & $\frac{c_{2}}{c_{1}} \cdot(k+1)$ & Lemma A.11 \\
ColPrev & 2 & $2 k+1$ & Lemma A.9 \\
ColIni & 2 & $2 k+1$ & Lemma A.10 \\
Rae & 1 & $k+1$ & Lemma 4.12 and 4.18 \\
PGI & $k+1$ & $\frac{(k+1)(k+5)}{4}$ & Corollary 4.19) \\
DP & $k+2$ & $\frac{k^{2}+6 k+9}{4}$ & Lemma 4.23 \\
Shift & $\frac{k+3}{2}$ & $\frac{k^{2}+3 k+2}{2}$ & Lemma A.3 \\
SDP & $2 k+1$ & $2 k^{2}+k+1$ & Lemma A.5 \\
\hline
\end{tabular}

TABLE 1. Quality functions for several power indices

4.4. A parameterized class of weighted games and negative results. In this section we construct a parametric example in order to obtain negative results for $p$-binomial semivalues and the Johnston index with respect to the existence of Alon-Edelman type results.

Definition 4.24. For integers $l, k, m, n$ with $1 \leq l \leq k-1, n \geq 1$, and $0 \leq m \leq n+1$, we define the simple game $v_{n, m}^{k, l}$ given by its set of minimal winning coalitions

$$
\{U \subseteq[k]:|U|=l, U \neq T\} \cup\{T \cup V: V \subseteq(k, k+n],|V|=m\},
$$

where $T=(k-l, k]$.

We remark that the number of players of $v_{n, m}^{k, l}$ is given by $n+k$ and not by $n$ (as everywhere else within this paper). The players come in at most three different types.

Lemma 4.25. For integers $l, k, m, n$ satisfying the restrictions from Definition 4.24 the simple game $v_{n, m}^{k, l}$ is weighted. 
Proof. For $m \notin\{0, n+1\}$ a weighted representation is given by $\left[q ; w_{1}=a, \ldots, w_{k-l}=a, w_{k-l+1}=\right.$ $\left.b, \ldots, w_{k}=b, w_{k+1}=1, \ldots, w_{n+k}=1\right]$, where $b=(l-2) m+n+1, a=(l-1) m+n+1$, and $q=l b+m=(l-1)^{2} m+l(n+1)$. For $m=n+1$ a weighted representation is given by $\left[q=l^{2}+1 ; w_{1}=l+1, \ldots, w_{k-l}=l+1, w_{k-l+1}=l, \ldots, w_{k}=l, w_{k+1}=0, \ldots, w_{n+k}=0\right]$ and for $m=0$ a weighted representation is given by $\left[q=l ; w_{1}=1, \ldots, w_{k-l}=1, w_{k-l+1}=\right.$ $\left.1, \ldots, w_{k}=1, w_{k+1}=0, \ldots, w_{n+k}=0\right]$.

The family of parametric games $v_{n, m}^{k, l}$ has several useful properties: As just mention the games weighted, i.e. belong to the most narrow class $\mathcal{T}_{n}$ of binary games that we are considering in this paper. With the help of the parameters $l, k, m, n$ one can, to some degree, control the power of the players in $[1, k-k]$ and of those in $(k, k+n]$. The games $v_{n, m}^{k, l}$ are almost $k$-pure, i.e. $T$ is the only coalition $S \subseteq[k]$ such that the reduced game $\mathcal{W}_{S}$ is a simple game. In other words, each shortening function can just modify coalitions of the form $T \cup V$, where $V \subseteq(k, k+n]$. Since in a $k$-pure game it only depends on $T$ whether the $T \cup V$ are winning or losing, there are just two possible choices. Turning $T \cup V$ into losing coalitions for all $V \subseteq(k, k+n]$ results in $v_{n, n+1}^{k, l}$ and turning $T \cup V$ into losing winning for all $V \subseteq(k, k+n]$ results in $v_{n, 0}^{k, l}$. For the special shortening function $k$-rounding we can explicitly state which case occurs:

Lemma 4.26. Let $\Gamma$ denote the operation of $k$-rounding and $l, k, m, n$ be integers satisfying the restrictions from Definition 4.24 Then we have

$$
\Gamma\left(v_{n, m}^{k, l}\right)=\left\{\begin{aligned}
v_{n, 0}^{k, l} & : \quad m \leq\lfloor n / 2\rfloor, \\
v_{n, n+1}^{k, l} \quad & : \text { otherwise. }
\end{aligned}\right.
$$

We want to study the absolute difference of individual power between the three games $v_{n, m}^{k, l}, v_{n, n+1}^{k, l}$, and $v_{n, 0}^{k, l}$ for the (at most) three types of players $[1, k-l],(k-l, j]$, and $(k, k+n]$. As an abbreviation we introduce:

Definition 4.27. For each positive power index $P: \mathcal{V}_{n} \rightarrow \mathbb{R}_{\geq 0}$ satisfying the null player property and each integers $l, k, m, n$ satisfying the restrictions from Definition 4.24 we set

$$
\begin{array}{rll}
\Delta_{1,2}^{1, P}=\left|P_{i}\left(v_{n, m}^{k, l}\right)-P_{i}\left(v_{n, n+1}^{k, l}\right)\right| & \forall i \in[1, k-l], \\
\Delta_{1,2}^{2, P}=\left|P_{i}\left(v_{n, m}^{k, l}\right)-P_{i}\left(v_{n, n+1}^{k, l}\right)\right| & \forall i \in(k-1, k], \\
\Delta_{1,2}^{3, P}=\left|P_{i}\left(v_{n, m}^{k, l}\right)-P_{i}\left(v_{n, n+1}^{k, l}\right)\right| & \forall i \in(k, k+n], \\
\Delta_{1,3}^{1, P}=\left|P_{i}\left(v_{n, m}^{k, l}\right)-P_{i}\left(v_{n, 0}^{k, l}\right)\right| & \forall i \in[1, k-l], \\
\Delta_{1,3}^{2, P}=\left|P_{i}\left(v_{n, m}^{k, l}\right)-P_{i}\left(v_{n, 0}^{k, l}\right)\right| & \forall i \in(k-1, k], \\
\Delta_{1,3}^{3, P}=\left|P_{i}\left(v_{n, m}^{k, l}\right)-P_{i}\left(v_{n, 0}^{k, l}\right)\right| & \forall i \in(k, k+n], \text { and } \\
\xi^{P}=\sum_{i=k+1}^{k+n} P_{i}\left(v_{n, m}^{k, l}\right) . &
\end{array}
$$

In other words, $\xi^{P}$ is the aggregated power of the players in $(k, k+n]$ of the game $v_{n, m}^{k, l}$. For $v_{n, n+1}^{k, l}$ and $v_{n, 0}^{k, l}$ the corresponding aggregated power equals zero so that we have $\xi^{P}=n \Delta_{1,2}^{3, P}=n \Delta_{1,3}^{3, P}$.

Our first aim is to show that there can be no Alon-Edelman type result for $p$-binomial semivalues with $p \neq \frac{1}{2}$ and $k$-rounding, i.e. the Banzhaf index is unique within the class of $p$-binomial semivalues with respect to this property. To this end we prove the following limit results in Subsection B.1.

Lemma 4.28. Let $P=\Psi^{p}$ and $k, l$ be integers with $1 \leq l \leq k-1$.

(1) For $m=\left\lceil\frac{n+1}{2}\right\rceil, p \in\left(\frac{1}{2}, 1\right)$ we have $\lim _{n \rightarrow \infty} \frac{\Delta_{1,2}^{1, P}}{\xi^{P}} \rightarrow \infty$ and $\lim _{n \rightarrow \infty} \frac{\Delta_{1,2}^{2, P}}{\xi^{P}} \rightarrow \infty$.

(2) For $m=\left\lfloor\frac{n}{2}\right\rfloor, p \in\left(0, \frac{1}{2}\right)$ we have $\lim _{n \rightarrow \infty} \frac{\Delta_{1,3}^{1, P}}{\xi^{P}} \rightarrow \infty$ and $\lim _{n \rightarrow \infty} \frac{\Delta_{1,2}^{3, P}}{\xi^{P}} \rightarrow \infty$.

(3) For $m \in\left\{\left\lceil\frac{n+1}{2}\right\rceil,\left\lfloor\frac{n}{2}\right\rfloor\right\}$ and $p \in(0,1) \backslash\left\{\frac{1}{2}\right\}$ we have $\lim _{n \rightarrow \infty} \xi^{P}=0$. 
We remark that (3) is not valid for $p=\frac{1}{2}$. Next we bound the overall $\|\cdot\|_{1}$-distance between $v_{n, m}^{k, l}$ and $v_{n, n+1}^{k, l}$ or $v_{n, 0}^{k, l}$ :

Lemma 4.29. For $p \in(0,1) \backslash\left\{\frac{1}{2}\right\}$ and integers $l, k, m, n$ satisfying the restrictions from Definition 4.24 we have

$$
\left|\sum_{i=1}^{n+k} \Psi_{i}^{p}\left(v_{n, m}^{k, l}\right)-\sum_{i=1}^{n+k} \Psi_{i}^{p}\left(v_{n, n+1}^{k, l}\right)\right| \leq k \cdot p^{l-1}(1-p)^{k-l-1}+\xi^{\Psi^{p}}
$$

and

$$
\left|\sum_{i=1}^{n+k} \Psi_{i}^{p}\left(v_{n, m}^{k, l}\right)-\sum_{i=1}^{n+k} \Psi_{i}^{p}\left(v_{n, 0}^{k, l}\right)\right| \leq k \cdot p^{l-1}(1-p)^{k-l-1}+\xi^{\Psi^{p}} .
$$

For the proof we refer the reader to Subsection B.1. By suitably choosing $k$ and $l$ we can achieve that the bound of Lemma 4.29 guarantees a small chance between $v_{n, m}^{k, l}$ and its $k$-rounding. $l=\left\lceil\frac{k}{2}\right\rceil$ and $k$ sufficiently large but fix will do the job. Next we can increase $n$ and apply Lemma 4.28 . Since the values $f_{1}(k), f_{2}(k)$ of the hypothetical quality functions would be finite and we can choose $\varepsilon=\xi^{P}$, an Alon-Edelman type result cannot exist for $k$-rounding.

theorem 4.30. For $k \in \mathbb{N}, p \in(0,1) \backslash\left\{\frac{1}{2}\right\}, \mathcal{T}_{n} \subseteq \mathcal{V}_{n}$ being compatible with $k$-rounding, no AlonEdelman type result for $\Psi^{p}$ and $k$-rounding can exist.

Since the changes can be made arbitrarily small, due to Lemma 4.29 , the corresponding statement for the normalized $p$-binomial semivalue is also valid. Having a closer look at the proofs of the technical bounds in Subsection B.1 we observe that the argumentation will break down if we slightly adopt the shortening function $k$-rounding.

Definition 4.31. For a given Boolean game $v=(\mathcal{W},[n])$, a real constant $p \in(0,1)$, and an integer $1 \leq k \leq n$ we denote by $v^{\prime}=(\mathcal{W},[n])$ the game that arises from $v$ as follows: for every $A \subseteq[k]$ we set $\mathcal{W}_{A}^{\prime}=\emptyset$ if $\left|\mathcal{W}_{A}\right| \leq p \cdot 2^{n-k}$ and $\mathcal{W}_{A}^{\prime}=2^{(k, n]}$ if $\left|\mathcal{W}_{A}\right|>p \cdot 2^{n-k}$. We call the mapping $\Gamma$ that maps $(v, k)$ to $v^{\prime}$ the $(p, k)$-rounding.

In this new notation our old $k$-rounding is denoted as $\left(\frac{1}{2}, k\right)$-rounding. There might be an intuition for this special choice. Taking as $p$ the probability for voting yes for each player, the expected number of winning coalitions in $\mathcal{W}_{A}$ would be $p \cdot 2^{n-k}$. In some sense one would compare the present situation of the reduced game to the expected situation and rounds with respect to this threshold. Admittedly, this interpretation might not carry very far or even be wrong. Nevertheless we state:

Conjecture 4.32. For $k \in \mathbb{N}, p \in(0,1), \mathcal{T}_{n} \subseteq \mathcal{V}_{n}$ being compatible with $(p, k)$-rounding, AlonEdelman type results for $\Psi^{p}$ and $(p, k)$-rounding do exist.

Although we have tested the validity of Conjecture 4.32 for several examples, it remains unclear, if just counting the number of winning coalitions in the respective reduces games is detailed enough so that $(p, k)$-rounding works as expected. We remark that at the very least, there are generalizations of the edge-isoperimetric inequality for the cube-graph with a binomial probability distribution with expectation $p$, see e.g. [27, 28].

We want to complete this subsection with the discussion of the Johnston index, which does, interestingly enough, not admit an Alon-Edelman type result for any shortening function. To this end we consider the games $v_{n, m}^{k, 1}$ and state the following inequalities :

Lemma 4.33. For integers $n=2 \tilde{n}+1 \geq 3$ odd, $l=1, m=\tilde{n}+1$, and $k \geq 2$ we have

$$
\begin{aligned}
\left\|\mathrm{JS}\left(v_{n, m}^{k, l}\right)-\mathrm{JS}\left(v_{n, n+1}^{k, l}\right)\right\|_{1} & \geq\left(k \cdot \frac{\sqrt{\tilde{n}}}{\sqrt{2}}+1\right) \xi^{\mathrm{JS}}, \\
\left\|\mathrm{JS}\left(v_{n, m}^{k, l}\right)-\mathrm{JS}\left(v_{n, 0}^{k, l}\right)\right\|_{1} & \geq\left((k-1) \cdot \frac{\sqrt{\tilde{n}}}{\sqrt{2}}+1\right) \xi^{\mathrm{JS}}, \text { and } \\
\lim _{\tilde{n} \rightarrow \infty} \frac{\xi^{\mathrm{JS}}}{\sum_{i=1}^{n+k} \mathrm{JS}_{i}\left(v_{n, m}^{k, l}\right)} & =0 .
\end{aligned}
$$


Having Lemma 4.33 at hand, whose proof is delayed to Subsection B.2, we can conclude that for the absolute Johnston index rounding up or down does not yield the desired approximation result for all $n \in \mathbb{N}$.

theorem 4.34. For $\mathcal{T}_{n} \subseteq \mathcal{V}_{n}$ being compatible with an arbitrary shortening function $\Gamma$ on $\mathcal{V}_{n}$, no Alon-Edelman type result for JS and $\Gamma$ can exist.

Theorem 4.34 leaves little room for an approximation result similar to Theorem 4.2, since our only restriction is that of using a shortening function. So in principle it may exist an approximation result but only with a more dramatic change of the game. For the normalized Johnston index $\widetilde{\mathrm{JS}}$ the following bounds let us draw a similar conclusion:

Lemma 4.35. For integers $n=2 \tilde{n}+1$ odd, $l=1, m=\tilde{n}+1$, and $k \geq 2$, where $\tilde{n}$ is sufficiently large, we have

$$
\begin{aligned}
\left\|\widehat{\mathrm{JS}}\left(v_{n, m}^{k, l}\right)-\widehat{\mathrm{JS}}\left(v_{n, n+1}^{k, l}\right)\right\|_{1} & \geq \frac{1}{5 k}, \\
\left\|\widehat{\mathrm{JS}}\left(v_{n, m}^{k, l}\right)-\widehat{\mathrm{JS}}\left(v_{n, 0}^{k, l}\right)\right\|_{1} & \geq \frac{1}{5 k}, \text { and } \\
\lim _{\tilde{n} \rightarrow \infty} \xi^{\widehat{\mathrm{JS}}} & =0 .
\end{aligned}
$$

\section{ILP FORMULATIONS FOR THE EXACT SOLUTION OF THE INVERSE POWER INDEX PROBLEM}

In this section we will develop a generic integer linear programming formulation for the exact solution of the inverse power index problem for power indices based on the idea of counting functions. They mimic and generalize the ideas from [46], where the Shapley-Shubik and the Banzhaf index have been treated.

5.1. The classes of underlying Boolean games. In order to model a Boolean game $v: 2^{N} \rightarrow\{0,1\}$ we introduce binary variables $x_{S} \in\{0,1\}$ for all coalitions $S \subseteq N$ with the meaning $x_{S}=v(S)$, i.e. $x_{S}=1$ for winning and $x-S=0$ for losing coalitions. The two conditions, $v(\emptyset)=0$ and $v(N)$, of Definition 2.1 can then be written as $x_{\emptyset}=0$ and $x_{N}=0$. Next we state the conditions to model the refinements of Boolean games described in Section 2 .

The incidence vectors $x_{S}$ of the winning coalitions of a Boolean game correspond to a simple game if the constraints

$$
x_{S \backslash\{i\}} \leq x_{S} \quad \forall \emptyset \neq S \subseteq N, i \in S
$$

are satisfied. For complete simple games we assume $1 \succeq 2 \succeq \cdots \succeq n$, i.e. the players are numbered from the most powerful to the least powerful. Here a set of sufficient conditions is given by

$$
\begin{aligned}
x_{S \cup\{k(S)+1\} \backslash\{k(S)\}} & \leq x_{S} \quad \forall \emptyset \neq S \subseteq N \backslash n \text { and } \\
x_{S \backslash\{n\}} & \leq x_{S} \quad \forall\{n\} \subseteq S \subseteq N,
\end{aligned}
$$

where $k(S)=\max \{i \mid i \in S\}$ denotes the maximum index of a player in coalition $S$. We remark that the inequalities (2) and (3) dominate the inequalities (1).

For weighted games we use the inequalities (2) and (3) for complete simple games and additionally introduce the variables $q, w_{1}, \ldots, w_{n} \in \mathbb{R}_{\geq 0}$ for the quota and the weights of the players. It is a well known fact that each weighted game admits a representation where all weights and the quota are integers. Being slightly less restrictive we assume a weighted representation where the weight of every winning coalition is at least one more than the weight of an arbitrary losing coalition, i.e. we assume $w(S) \geq q$ for all winning coalitions $S \subseteq N$ and $w(T) \leq q-1$ for all losing coalitions $T \subseteq N$. To this end we can require that the quota is at least one:

$$
q \geq 1 \text {. }
$$

The assumed ordering of the players of the underlying complete simple game induces an ordering on the weights:

$$
w_{i} \leq w_{i+1} \quad \forall 1 \leq i \leq n-1
$$


It remains to interlink the weights $w_{i}$ with the incidences $x_{S}$ :

$$
\begin{gathered}
q-\left(1-x_{S}\right) \cdot M-\sum_{i \in S} w_{i} \leq 0 \quad \forall S \subseteq N \text { and } \\
q+x_{S} \cdot M-\sum_{i \in S} w_{i} \geq 1 \quad \forall S \subseteq N,
\end{gathered}
$$

where $M$ is an suitably large constant fulfilling $M \geq \sum_{i=1}^{n} w_{i}$. (According to [62, Theorem 9.3.2.1] we may choose $M=4 n\left(\frac{n+1}{4}\right)^{(n+1) / 2}$.) For a winning coalition $S$, i.e. $x_{S}=1$, inequality $(6)$ is equivalent to $\sum_{i \in S} w_{i} \geq q$. Similarly, for a losing coalition $T$, i.e. $x_{T}=0$, inequality 77 is equivalent to $\sum_{i \in S} w_{i} \leq q-1$. The two other combinations are equivalent to automatically true inequalities if $M$ is chosen sufficiently large. More concretely, for a winning coalition $S$ inequality $(7)$ is equivalent to $\sum_{i \in S} w_{i} \leq q-1+M$, which in turn is true due to $M \leq q-1+M$ and $M \geq \sum_{i=1}^{n} w_{i}$. For each losing coalition $T$ inequality $(6)$ is equivalent to $\sum_{i \in S} w_{i} \geq q-M$, which is true if we additionally require $q \leq M$.

Sufficient conditions for proper games can be easily stated as

$$
x_{S}+x_{N-S} \leq 1 \quad \forall S \subseteq N,|S| \leq n / 2 .
$$

Similarly we have

$$
x_{S}+x_{N-S} \geq 1 \quad \forall S \subseteq N,|S| \leq n / 2
$$

for strong games. For constant-sum games we can take inequalities (8) and (9).

5.2. Power indices based on a counting function. Let $\mathcal{V}_{n}$ be a class of binary voting games on $n$ players. In the previous subsection we have provided ILP formulations for all types of binary voting games described in Section 2 and remark that other classes require, expectably relatively easy and standard, adaptations. For the power index we here assume that it is based on a counting function $C: \mathcal{V}_{n} \times 2^{N} \times N \rightarrow \mathbb{R}_{\geq 0}^{n}$. So we introduce the nonnegative real variables $y_{i, S} \in \mathbb{R}_{\geq 0}$, with the interpretation $y_{i, S}=C_{i}(v, S)$, for all $i \in N, S \subseteq N$ and a game $v \in \mathcal{V}_{n}$ represented by the $x_{S}$ and the constraints from Subsection 5.1. We model the induced power index $P: \mathcal{V}_{n} \rightarrow \mathbb{R}_{\geq 0}^{n}$ by the variables $p_{i} \in \mathbb{R}_{\geq 0}$ and the constraints

$$
p_{i}=\sum_{S \subseteq N} y_{i, S}
$$

for all $i \in N$. The constraints for the values $y_{i, S}=C_{i}(v, S)$ depend on the precise definition of the respective counting function. For the power indices introduced in Section 3, which are based on counting functions, they can easily be stated, since the underlying concepts of e.g. winning, losing, minimal winning, or shift-minimal winning coalitions can be directly expressed using the binary variables $x_{S}$.

For the Shapley-Shubik index, given by the counting function

$$
C_{i}^{\mathrm{SSI}}(v, S)=\left\{\begin{aligned}
\frac{(|S|-1) !(n-|S|) !}{n !} & : \quad i \in S, v(S)=1, v(S \backslash\{i\})=0, \\
0 & : \text { otherwise, }
\end{aligned}\right.
$$

and a subclass $\mathcal{V}_{n} \subseteq \mathcal{S}_{n}$ of simple games we can state $y_{i, S}=\frac{(|S|-1) !(n-|S|) !}{n !} \cdot\left(x_{S}-x_{S \backslash\{i\}}\right)$ for all $\{i\} \subseteq S \subseteq N$ and $y_{i, S}=0$ for all $S \subseteq N \backslash\{i\}$. If we cannot guarantee $x_{S}-x_{S \backslash\{i\}} \geq 0$, i.e. when the game described by the $x_{S}$ is not simple, we have to model the first set of constraints differently:

$$
\begin{aligned}
& y_{i, S} \leq \frac{(|S|-1) !(n-|S|) !}{n !} \cdot x_{S}, \\
& y_{i, S} \leq \frac{(|S|-1) !(n-|S|) !}{n !} \cdot\left(1-x_{S \backslash\{i\}}\right), \\
& y_{i, S} \geq \frac{(|S|-1) !(n-|S|) !}{n !} \cdot\left(x_{S}-x_{S \backslash\{i\}}\right)
\end{aligned}
$$

for all $\{i\} \subseteq S \subseteq N$. If either $i \notin S, v(S)=x_{S}=0$, or $v(S \backslash\{i\})=x_{S \backslash\{i\}}=1$, then we clearly have $y_{i, S}=0$ due to $y_{i, S} \geq 0$. From the third set of the above inequalities we conclude 
$y_{i, S}=\frac{(|S|-1) !(n-|S|) !}{n !}$ for the case where $v(S)=x_{S}=1$ and $v(S \backslash\{i\})=x_{S \backslash\{i\}}=0$, since $y_{i, S} \leq \frac{(|S|-1) !(n-|S|) !}{n !} \cdot x_{S}$.

Generally, we remark that there is a rich modeling theory for ILPs and we want to briefly give a few, in our context, relevant ideas. Let $a \in\{0,1\}$ be a variable representing a logical value. Assume that we want to use a conditional inequality $f(b) \leq c$ just for the cases where $a=1$ and drop it for $a=0$. Using a sufficiently large real constant $M$ we can formulate this situation as

$$
f(b) \leq c+(1-a) \cdot M .
$$

This technique is called Big-M method and we have already seen its application in the case of weighted games in the previous subsection. Since $f(b) \geq c$ is equivalent to $-f(b) \leq-c$ and $f(b)=c$ is equivalent to $f(b) \leq c$ and $f(b) \geq c$, all types of conditional linear inequalities can be modeled. For all logic operations, i.e. arbitrary combinations of and, or, and negation not of a finite number of Boolean logical values, there exists a set of inequalities to model the corresponding logic gate.

In our example for the SSI, things become more smoothly if we additionally introduce the binary variables $y_{i, S}^{\prime} \in\{0,1\}$ and set $y_{i, S}=\frac{(|S|-1) !(n-|S|) !}{n !} \cdot y_{i, S}^{\prime}$.

We provide a list of ILP models for the power indices of Section 3 , which are based on counting functions, in appendix C. We have to remark that such ILP models can normally easily be obtained for the absolute versions of the power indices. For the respective normalized versions we give a general reduction to a sequence of ILPs in the next subsection. In some cases it is possible to reformulate, to be more precisely to exactly linearize, a linear fractional term to several linear constraints, see e.g. [55].

5.3. Measurement of the deviation. Suppose we are given a target vector $\sigma=\left(\sigma_{1}, \ldots, \sigma_{n}\right)$ of the desired power distribution. Given a certain power index $P$, a class of binary voting games $\mathcal{V}_{n}$, and a norm $\|\cdot\|$, the inverse power index problem asks for a game $v \in \mathcal{V}_{n}$ such that the deviation $\|P(v)-\sigma\|$ from the desired power distribution is minimized. The details for the class of binary games and power indices have been given in the previous subsections. Now we go into the details for the selectable norms. When staying in the class of ILPs we have to, more or less, restrict ourselves onto the sum of absolute values $\|\cdot\|_{1}$ and the maximum norm $\|\cdot\|_{\infty}$.

Using more general optimization problems as a framework we can also write down other norms easily or eventually solve the resulting problems with the corresponding optimization algorithms and software packages. In this context ILPs are far more innocent than their generalization to MINLPs (Mixed Integer Nonlinear Programming).

In both treated cases we need the partial expression $\left|p_{i}-\sigma_{i}\right|$ for all $i \in N$. To this end we introduce the nonnegative real variables $\delta_{i}$ and the inequalities

$$
\begin{aligned}
p_{i}-\sigma_{i} & \leq \delta_{i} \text { and } \\
\sigma_{i}-p_{i} & \leq \delta_{i}
\end{aligned}
$$

for all $i \in N$. With this, all feasible solutions satisfy $\delta_{i} \geq\left|p_{i}-\sigma_{i}\right|$. For the $\|\cdot\|_{1}$ norm we can minimize the target function

$$
\sum_{i=1}^{n} \delta_{i}
$$

so that we have $\delta_{i}=\left|p_{i}-\sigma_{i}\right|$ for each minimal solution. For the $\|\cdot\|_{\infty}$ norm we can identify the $\delta_{i}$, i.e. set $\delta=\delta_{1}=\cdots=\delta_{n}$ and minimize $\delta$, so that we have $\delta=\left|p_{i}-\sigma_{i}\right|$ for each minimal solution and all $i \in N$.

Given that the three parts of an inverse power index problem can be expressed linearly with integer (and real) variables, we can make use of one of the many available software packages for ILPs. For our practical computations we have used the software CPLEX version 12.4 from IBM ILOG.

Once we can formulate a certain inverse power index problem with power index $P$, class $\mathcal{V}_{n}$, and norm $\|\cdot\|$ as an ILP, we can solve the corresponding problem with the normalized power index $\widehat{P}$ instead of $P$ by a sequence of ILPs. Actually we have to express

$$
\left|\frac{p_{i}}{\sum_{j=1}^{n} p_{j}}-\sigma_{i}\right|=\delta_{i}
$$


linearly (for all $i \in N$ ). Since $p_{i} \geq 0$, we can multiply both sides with $\sum_{j=1}^{n} p_{j}$ and obtain

$$
\left|p_{i}-\sigma_{i} \cdot \sum_{j=1}^{n} p_{j}\right|=\delta_{i} \cdot \sum_{j=1}^{n} p_{j} .
$$

The absolute value on the left hand side can be easily linearized as show before. Unfortunately the right hand side contains quadratic terms, i.e. the summands $\delta_{i} \cdot p_{j}$ depend on two variables. Since none of the two variable types is binary, there is no standard linearization available. As a workaround we introduce $\delta_{i}^{\prime}=\delta_{i} \cdot \sum_{j=1}^{n} p_{j}$ as new nonnegative real variables for all $i \in N$. With this, the corresponding constraints are given by

$$
\begin{aligned}
& p_{i}-\sigma_{i} \cdot \sum_{j=1}^{n} p_{j} \leq \delta_{i}^{\prime} \text { and } \\
& \sigma_{i} \cdot \sum_{j=1}^{n} p_{j}-p_{i} \leq \delta_{i}^{\prime}
\end{aligned}
$$

for all $i \in N$. Minimizing $\sum_{i=1}^{n} \delta_{i}^{\prime}$ (for $\|\cdot\|_{1}$ ) or $\delta^{\prime}=\delta_{1}^{\prime}=\cdots=\delta_{n}^{\prime}$ (for $\|\cdot\|_{\infty}$ ) would not yield the desired result. Instead we introduce a numerical parameter $\alpha$ and the constraint

$$
t\left(\delta_{1}^{\prime}, \ldots, \delta_{n}^{\prime}\right) \leq \alpha \cdot \sum_{j=1}^{n} p_{j},
$$

where $t\left(\delta_{1}, \ldots, \delta_{n}\right)$ is the original target function. We completely drop the old target function and obtain a so called feasibility problem, which can be solved with the same methods as ILPs including a target function. The interpretation of $\alpha$ is as follows: If, for a given $\alpha$, the corresponding ILP contains a feasible solution, then there exists a solution of $\|P(v)-\sigma\| \leq \alpha$ and each game $v$ being described by such a feasible solution satisfies this inequality. If otherwise the set of feasible solutions is empty, we have $\|P(v)-\sigma\|>\alpha$ for all $v \in \mathcal{V}_{n}$.

Initially we generally know that the minimum value of $\|P(v)-\sigma\|$ is contained in the interval $[0, \infty)$. Using a bisection algorithm for this interval and $\alpha$, we obtain a sequence $\left[l_{1}, r_{1}\right] \supseteq \cdots \supseteq$ $\left[l_{m}, r_{m}\right]$ of intervals of decreasing length such that the minimum value of $\|P(v)-\sigma\|$ is contained in $\left[l_{j}, r_{j}\right]$ for all $1 \leq j \leq m$. Thus we can determine the minimum possible deviation (and a corresponding game) up to each given precision. If $\mathcal{V}_{n}$ is finite there are only finitely many attainable power distributions $P(v)$, so that this convergence suffices to obtain the exact solution after a finite number of iterations, i.e. there exists a problem-dependent upper bound on the length of $\left[l_{m}, r_{m}\right]$ which suffices to conclude that the best found solution is already optimal. For the Banzhaf index the technical details have been described in [46] and, in more detail, in [48].

\section{POWER Distributions Which ARE NOT CONCENTRATED ON THE FIRST $k$ PLAYERS}

Let $\sigma=\left(\sigma_{1}, \ldots, \sigma_{n}\right) \in \mathbb{R}_{>0}^{n}$ be a desired power vector with $\|\sigma\|_{1}=1$. Given a power index $P$, a norm $\|\cdot\|$, and a class $\mathcal{V}_{n}$ of binary games on $n$ players, the inverse power index asks for a game $v \in \mathcal{V}_{n}$ minimizing the deviation $\|P(v)-\sigma\|$. In the previous section we have described an exact integer linear programming approach for those power indices from Section 3 , which are based on counting functions. For a concrete instance of the inverse power index problem we may eventually find the exact solution algorithmically in a reasonable amount of time.

Are more general statements achievable? If the number $n$ of players is small, there is only a relatively small finite number of games in $\mathcal{V}_{n}$, so that $\min _{v \in \mathcal{V}_{n}}\|P(v)-\sigma\|$ may not become too small. Whether $\min _{v \in \mathcal{V}_{n}}\|P(v)-\sigma\|$ is rather small or rather large can be decided by just looking at the class of $k$-pure games in $\mathcal{V}_{n}$ with the aid of the Alon-Edelman type bounds from Section 4 . provided that most of the power of $\sigma$ is concentrated on the first $k$ coordinates, i.e. $\sum_{i=k+1} \sigma_{i} \ll 1$. Despite this rather vague description, things can be made very precise by stating bounds in terms on a parameter $\varepsilon$, with $\sum_{i=k+1} \sigma_{i} \leq \varepsilon$. Of course we cannot say much more, since there are power distributions like e.g. $\sigma_{n}=(0.75,0.25,0 \ldots, 0)$ which are rather hard to approximate for most power indices and others like $\sigma^{\prime}=(0.5,0.5,0 \ldots, 0)$, which can be perfectly met. 
Is the assumption that most of the power is concentrated on the first $k$ components a realistic assumption that is commonly satisfied in practice? Instead of an answer we aim to classify all vectors of the unit simplex into different types of desired power distributions $\sigma$. To this end let us call coordinates $i$, where $\sigma_{i}$ is relatively small, oceanic. Those coordinates, where $\sigma_{i}$ is relatively large, are called islands. If we have a sequence $\sigma^{(n)}$ of desired power distributions with increasing number of players for all $n \in \mathbb{N}$, we can made this more precise immediately: We call coordinate $i$ oceanic iff $\lim _{n \rightarrow \infty} \sigma_{i}^{(n)}=0$ and islands iff there exists a lower bound $u_{i}>0$ such that $\sigma_{i}^{(n)} \geq u_{i}$ for all sufficiently large $n$. In principle there may be coordinates which are neither oceanic nor islands, e.g. we may have $\sigma_{i}^{(n)}=1 / 7$ for odd $n$ and $\sigma_{i}^{(n)}=\frac{1}{n^{2}}$ for even $n$. Those cases may be considered as not well behaved and we ignore them, if not all coordinates are either oceanic or islands.

Now let $k$ denote the number of islands of such a sequence. If the aggregated desired power of the oceanic players is rather small or even tends to zero, we can apply the Alon-Edelman type results. The two other cases are that we have no, i.e. $k=0$, islands or the aggregated desired power of the oceanic players is non-vanishing. For simplicity, we assume in the latter case that the desired power of the ocean is roughly given by a real constant $\alpha$. In both cases the so-called limit results for power indices give precise theoretical predictions about the power distribution when considering weighted games with weights $w_{i}$ equal to $\sigma_{i}$.

Let us first consider the case where no island is present, i.e. $k=0$ or in other words, where $\max _{i} \sigma_{i}^{(n)}$ tends to zero as $n$ increases. The strongest statements are the so-called Penrose's limit (type) theorems, see [69]: Under certain technical conditions we have

$$
\lim _{n \rightarrow \infty} \frac{P_{i}\left(\left[q ; \sigma_{1}^{(n)}, \ldots, \sigma_{i}^{(n)}, \ldots\right]\right)}{w_{i}}=1 .
$$

Typical assumptions are that $\left\|\sigma^{(n)}\right\|_{\infty}$ tends to zero, and that the entries $\sigma_{i}^{(n)}$ only take a finite number of different values for each $n$ and that the number of occurrences of each type increases with $n$-socalled replicative chains of weighted games. For the Banzhaf index such a result was proven for quota $q=\frac{1}{2}$ and for the Shapley-Shubik index for all fixed quotas $q \in(0,1)$, see [56]. But there are also cases where such a strong result does not hold, see e.g. [13, 57]. The technical assumption of replicative chains can be dropped if relative convergence of the fractions in Equation (17) is replaced by absolute convergence in e.g. the $\|\cdot\|_{1}$-norm. [64, Theorem 9.8] states, in different notation

$$
\lim _{n \rightarrow \infty}\left\|\operatorname{SSI}\left(v^{(n)}\right)-\sigma^{(n)}\right\|_{1}=0
$$

where the weights of the weighted games $v^{(n)}$ can be taken as $\sigma^{(n)}$ and quota $q$ can be taken almost arbitrarily, i.e. there should be no cluster point at either 0 or 1 . We remark that generally an absolute limit result like Equation (18) implies a relative limit result like Equation (17) provided that replicative chains are considered.

A big drawback of those limit results might be that they cannot be directly applied to a given single desired power distribution $\sigma$. Whenever there are concrete error bounds we can say something for a given single desired power distribution $\sigma$. Recently such a bound has been given in [49] for the nucleolus:

$$
\|\operatorname{Nuc}([q ; w])-w\|_{1} \leq \frac{2 \cdot \max _{1 \leq i \leq n} w_{i}}{\min (q, 1-q)},
$$

for all $q \in(0,1)$ and $w \in \mathbb{R}_{\geq 0}^{n}$ with $\|w\|_{1}=1$. For the SSI such bounds should be hidden in the proofs of the technical lemmas of [64].

In general we cannot expect much more than an inequality similar to $19 p$ : Let $P$ be a symmetric, efficient and positive power index satisfying the null player property. For $\sigma^{(n)}=\frac{1}{2 n-1} \cdot(2, \ldots, 2,1)$ we have

$$
\left\|P\left(\left[q ; \sigma^{(n)}\right]\right)-\sigma^{(n)}\right\|_{1} \geq \frac{2}{2 n-1} \cdot \frac{n-1}{n}
$$

and $\max _{i} \sigma_{i}^{(n)}=\frac{2}{2 n-1}$ for all $n$, see [48]. So for this special sequence of desired power distributions, taking the desired power as weights yields a $\theta\left(\frac{1}{n}\right)$-error, which is still considerably large for medium sized constitutions like e.g. $n=27$ or 28 . For $P=\mathrm{Bz}$ we can remark that solving the inverse power 
index yields slightly better bounds than Inequality $(20)$ within the class $\mathcal{T}_{n}$ and an exact solution within the class of simple games for all $6 \leq n \leq 18$, see [48, Table 9].

For the remaining case of a small but positive number of islands and a non-vanishing ocean there are limit results too. In [73, Theorem 1] an approximation formula with an estimate for the rate of convergence was given. Here the assumptions on the weight distribution within the ocean are rather mild. Interestingly enough, provided a small variance of the weight distribution within the ocean the error term is of the form $\theta\left(\frac{1}{n}\right)$. If all oceanic players have the same weight, then there is a similar result for the Banzhaf index, which fails to be true for asymmetric weight distributions within the ocean, see [26]. Some first results in that direction for the nucleolus can be found in [36].

\section{CONCLUSION AND FUTURE WORK}

Inspired by the seminal work of [1], we have considered Alon-Edelman type results for most of the known power indices. It turned out that for the power indices KB, PHI, and Chow it makes no sense to ask for Alon-Edelman type results, since the respective power indices do not admit power distributions where most of the power is concentrated on a small number of players. For the other considered power indices such a concentration is in principle possible. Nevertheless, there can be no such result for the Johnston index, which we have shown by analytical power index calculations for a certain class of parameterized weighted voting games, which might be interesting in its own right. For $p$-binomial semivalues and $k$-rounding we similarly have obtained a negative result. On the other hand we conjecture that there exists an Alon-Edelman type result for $p$-binomial semivalues with $(k, p)$-rounding. The cases of the Shapley-Shubik index and more general semivalues are left open. In order to classify and represent power indices in a unified way, we have introduced the concept of power indices based on counting functions. Except the nucleolus and the MSR index, due to somewhat global properties, all of the presented power indices of this paper, admit such a representation. For those power indices based on counting functions, we have provided a theoretical and notational framework to formulate Alon-Edelman type results, if they exist at all, in a unified and more or less compact way.

We are pretty sure that some of our bounds for the quality functions in Table 1 can be improved. It would also be interesting to construct worst case examples showing how far our estimates are from the real truth. In order to study the existence question of Alon-Edelman type results for the nucleolus or the MSR index possibly other techniques are necessary.

Maybe the concept of counting functions can be fruitfully used in different contexts in order to unify approaches for several power indices. Examples might be generating function algorithms to compute values of power indices for weighted voting games or the design of new, so far missed, power indices, see [2].

Since $k$-rounding is the right shortening function for most power indices which admit an AlonEdelman type result, it would be interesting to study further of its theoretic properties. We have shown that $k$-rounding preserves weightedness. What about the generalizations? Does $k$-rounding preserve the dimension or stays within the class of roughly weighted, $\alpha$-roughly weighted ${ }^{24}$. or homogeneous games?

In the context of Alon-Edelman type results, of course one may find it interesting to enlarge the class of binary voting games to games with several levels of approval in the input and output. If the number of option tends to infinity we end up with continuous models, see e.g. [47]. Also the $\|\cdot\|_{1}$ in the present Alon-Edelman type results may be replaced by different norms.

Alon-Edelman type results allow negative approximation results by reducing the $n$ player case to the $k$ player case, provided that the desired power is concentrated only on a few players. As a counterpart we have outlined the known theory of limit results for power indices. For our purposes it would be very valuable if some of these results could be turned into precise error estimates and possibly generalized to larger classes of power indices.

From the practical point of view we have presented a general exact algorithmic approach for the inverse power index problem for power indices based on counting functions using integer linear programming. Any application of more sophisticated techniques from integer linear programming would

\footnotetext{
${ }^{24}$ See [32] and [37], where three hierarchies of simple games have been introduced.
} 
be beneficial to shift the computational limits of this approach, which generally is NP complete so that exact solutions can be expected only for rather small numbers of players.

[1] close by mentioning that they began the study which vectors in the unit simplex can be closely approximated by Banzhaf vectors of simple games. We generalize their question to the list of known power indices and important subclasses of simple games. We agree that Alon-Edelman type results seem to be unable to provide a complete solution. If power has to be attained for regions, i.e. collections of players, instead of single players, one can well approximate any distribution, as shown for the special case of the Banzhaf index in [1, Proposition 3.1]. A similar statement is obviously true for all symmetric, positive and efficient power indices, which satisfy the null player property.

\section{REFERENCES}

[1] Alon, N. and Edelman, P. (2010). The inverse Banzhaf problem. Soc. Choice Welf., 34(3):371-377.

[2] Alonso-Meijide, J. and Freixas, J. (2010). A new power index based on minimal winning coalitions without any surplus. Decision Support Systems, 49(1):70-76.

[3] Alonso-Meijide, J., Freixas, J., and Molinero, X. (2012). Computation of several power indices by generating functions. Appl. Math. Comput., 219(8):3395-3402.

[4] Aumann, R. and Hart, S., editors (2002). Handbook of game theory with economic applications. Vol. 3. Handbooks in Economics. 11. Amsterdam: Elsevier. xxx, p. 1522-2351.

[5] Banzhaf, J. (1965). Weighted voting doesn't work: A mathematical analysis. Rutgers Law Rev., 19:317-343.

[6] Bertini, C., Freixas, J., Gambarelli, G., and Stach, I. (2013). Comparing power indices. Int. Game Theory Rev., 15(2).

[7] Bertini, C., Gambarelli, G., and Stach, I. (2008). A public help index. In Braham, M. and Steffen, F., editors, Power, freedom, and voting, pages 83-98. Springer.

[8] Bolger, E. (1986). Power indices for multicandidate voting games. Internat. J. Game Theory, 15(3):175-186.

[9] Braham, M. e. and Steffen, F. e. (2008). Power, freedom, and voting. Essays in honour of Manfred J. Holler. Papers presented at the Festschrift conference, Hamburg, Germany, August 17-20, 2006. Berlin: Springer. xiv, 438 p.

[10] Brams, S., Kilgour, D., and Affuso, P. (1989). Presidential power: A game-theoretic analysis. In Brace, P., Harrington, C., and King, G., editors, The Presidency in American Politics, pages 55-74. New York University Press.

[11] Brams, S. and Lake, M. (1978). Power and satisfaction in a representative democracy. In Ordenshook, P. C., editor, Game Theory and Political Science, pages 529-562. New York University Press.

[12] Branzei, R., Dimitrov, D., and Tijs, S. (2008). Models in cooperative game theory. 2nd revised ed. Berlin: Springer. xi, 203 p.

[13] Chang, P.-L., Chua, V., and Machover, M. (2006). LS Penrose's limit theorem: Tests by simulation. Math. Social Sci., 51(1):90-106.

[14] Chow, C.-K. (1961). On the characterization of threshold functions. In Proceedings of the Second Annual Symposium on Switching Circuit Theory and Logical Design, SWCT 1961, FOCS 1961, pages 34-38. IEEE.

[15] Coleman, J. (1971). Control of collectivities and the power of a collectivity to act. In B.Lieberman, editor, Social Choice, pages 269-300. Gordon and Breach.

[16] Colomer, J. and Martinez, F. (1995). The paradox of coalition trading. Journal of Theoretical Politics, 7(1):41-63.

[17] De, A., Diakonikolas, I., Feldman, V., and Servedio, R. (2012a). Nearly optimal solutions for the chow parameters problem and low-weight approximation of halfspaces. In Proceedings of the 44th symposium on Theory of Computing, STOC '12, pages 729-746, New York, USA. ACM.

[18] De, A., Diakonikolas, I., and Servedio, R. (2012b). The inverse Shapley value problem. In Automata, Languages, and Programming, pages 266-277. Springer.

[19] de Keijzer, B., Klos, T., and Zhang, Y. (2010). Enumeration and exact design of weighted voting games. In Proceedings of the 9th International Conference on Autonomous Agents and Multiagent Systems: Volume 1, pages 391-398. International Foundation for Autonomous Agents and Multiagent Systems.

[20] De Keijzer, B., Klos, T., and Zhang, Y. (2012). Solving weighted voting game design problems optimally: Representations, synthesis, and enumeration. arXiv preprint 1204.5213.

[21] Deegan Jr, J. and Packel, E. (1978). A new index of power for simple n-person games. Internat. J. Game Theory, 7(2):113-123.

[22] Dragan, I. (2005). On the inverse problem for semivalues of cooperative TU games. Int. J. Pure Appl. Math., 22(4):545-561.

[23] Dragan, I. (2012). On the inverse problem for multiweighted Shapley values of cooperative TU games. Int. J. Pure Appl. Math., 75(3):279-287.

[24] Dragan, I. (2013). The inverse problem for binomial semivalues of cooperative TU games. In Game Theory and Management. Proceedings of the Seventh International Conference Game Theory and Management/Editors Leon A. Petrosyan and Nikolay A. Zenkevich.-SPb.: Graduate School of Management SPbU, 2013.-274 p., volume 26, page 72.

[25] Dubey, P., Neyman, A., and Weber, R. (1981). Value theory without efficiency. Math. Oper. Res., 6(1):122-128.

[26] Dubey, P. and Shapley, L. (1979). Mathematical properties of the Banzhaf power index. Math. Oper. Res., 4(2):99-131.

[27] Ellis, D. (2011). Almost isoperimetric subsets of the discrete cube. Combin. Probab. Comput., 20(3):363-380.

[28] Falik, D. and Samorodnitsky, A. (2007). Edge-isoperimetric inequalities and influences. Combin. Probab. Comput., 16(5):693-712. 
[29] Felsenthal, D. and Machover, M. (1998). The measurement of voting power: Theory and practice, problems and paradoxes. Cheltenham: Edward Elgar. xviii, 322 p.

[30] Felsenthal, D. and Machover, M. (2005). Voting power measurement: a story of misreinvention. Soc. Choice Welf., 25(2-3):485-506.

[31] Freixas, J. and Kaniovski, S. (2014). The minimum sum representation as an index of voting power. European J. Oper. Res., 233(3):739-748.

[32] Freixas, J. and Kurz, S. (2014a). On $\alpha$-roughly weighted games. Internat. J. Game Theory. (to appear).

[33] Freixas, J. and Kurz, S. (2014b). On minimum integer representations of weighted games. Math. Social Sci., 67:9-22.

[34] Freixas, J. and Zwicker, W. (2003). Weighted voting, abstention, and multiple levels of approval. Soc. Choice Welf., 21(3):399-431.

[35] Freixas, J. and Zwicker, W. (2009). Anonymous yes-no voting with abstention and multiple levels of approval. Games Econ. Behav., 67(2):428-444.

[36] Galil, Z. (1974). The nucleolus in games with major and minor players. Internat. J. Game Theory, 3(3):129-140.

[37] Gvozdeva, T., Hemaspaandra, L., and Slinko, A. (2013). Three hierarchies of simple games parameterized by "resource" parameters. International Journal of Game Theory, 42(1):1-17.

[38] Holler, M. (1982). Forming coalitions and measuring voting power. Political studies, 30(2):262-271.

[39] Holler, M. (1998). Two stories, one power index. Journal of Theoretical Politics, 10(2):179-190.

[40] Holler, M. and Packel, E. (1983). Power, luck and the right index. Zeitschrift für Nationalökonomie, 43(1):21-29.

[41] Imrie, R. (1973). The impact of the weighted vote on representation in municipal governing bodies of New York State. Ann. New York Acad. Sci., 219(1):192-199.

[42] Isbell, J. (1958). A class of simple games. Duke Math. J., 25:423-439.

[43] Johnston, R. (1978). On the measurement of power: Some reactions to Laver. Environment and Planning A, 10(8):907-914.

[44] König, T. and Bräuninger, T. (2001). Decisiveness and inclusiveness: Intergovernmental choice of European decision rules. In Holler, M. J. and Owen, G., editors, Power indices and coalition formation, pages 273-290. Kluwer.

[45] Kurz, S. (2012a). On minimum sum representations for weighted voting games. Ann. Oper. Res., 196(1):361-369.

[46] Kurz, S. (2012b). On the inverse power index problem. Optimization, 61(8):989-1011.

[47] Kurz, S. (2014). Measuring voting power in convex policy spaces. Economies. to appear, arXiv preprint 1312.5936.

[48] Kurz, S. and Napel, S. (2014). Heuristic and exact solutions to the inverse power index problem for small voting bodies. Ann. Oper. Res., pages 1-27. (to appear).

[49] Kurz, S., Napel, S., and Nohn, A. (2014). The nucleolus of large majority games. Econom. Lett. to appear, arXiv preprint 1310.0004.

[50] Kurz, S. and Tautenhahn, N. (2013). On Dedekind's problem for complete simple games. Internat. J. Game Theory, 42(2):411-437.

[51] Laruelle, A. and Valenciano, F. (2011). Voting and collective decision-making. Bargaining and power. Reprint of the 2008 hardback ed. Cambridge: Cambridge University Press. xvii, 184 p.

[52] Laruelle, A. and Valenciano, F. (2013). Voting and power. In Power, Voting, and Voting Power: 30 Years After, pages 137-149. Springer.

[53] Laruelle, A. and Widgrén, M. (1998). Is the allocation of voting power among EU states fair? Public Choice, 94(34):317-339.

[54] Le Breton, M., Montero, M., and Zaporozhets, V. (2012). Voting power in the EU Council of Ministers and fair decision making in distributive politics. Math. Social Sci., 63(2):159-173.

[55] Liberti, L. (2007). Techniques de reformulation en programmation mathématique. Thèse d'Habilitation à diriger des Recherches, Université Paris IX - Dauphine. 93 pages, available at http://www.lix.polytechnique.fr/ liberti/hdr.pdf

[56] Lindner, I. and Machover, M. (2004). LS Penrose's limit theorem: proof of some special cases. Math. Social Sci., 47(1):37-49.

[57] Lindner, I. and Owen, G. (2007). Cases where the Penrose limit theorem does not hold. Math. Social Sci., 53(3):232_ 238.

[58] Malawski, M. (2004). "Counting" power indices for games with a priori unions. Theory and Decision, 56(1-2):125140.

[59] Milnor, J. and Shapley, L. (1978). Values of large games II: Oceanic games. Math. Oper. Res., 3(4):290-307.

[60] Montero, M. (2006). Noncooperative foundations of the nucleolus in majority games. Games Econ. Behav., 54(2):380397.

[61] Montero, M. (2013). On the nucleolus as a power index. In Power, Voting, and Voting Power: 30 Years After, pages 283-299. Springer.

[62] Muroga, S. (1971). Threshold logic and its applications. Wiley-Interscience, a Division of John Wiley \&amp; Sons, Inc., New York, XIV, 478 p.

[63] Nevison, C., Zicht, B., and Schoepke, S. (1978). A naive approach to the Banzhaf index of power. Behavioral Science, 23(2):130-131.

[64] Neyman, A. (1982). Renewal theory for sampling without replacement. Ann. Prob., pages 464-481.

[65] Nurmi, H. (1980). Game theory and power indices. Zeitschrift für Nationalökonomie, 40(1-2):35-58.

[66] Nurmi, H. (1982). The problem of the right distribution of voting power. In Power, Voting, and Voting Power, pages 203-212. Springer.

[67] O'Donnell, R. and Servedio, R. (2011). The chow parameters problem. SIAM J. Comput., 40(1):165-199. 
[68] Papayanopoulos, L. (1983). On the partial construction of the semi-infinite Banzhaf polyhedron. In Fiacco, A. and Kortanek, K., editors, Semi-Infinite Programming and Applications, volume 215 of Lecture Notes in Economics and Mathematical Systems, pages 208-218. Springer Berlin Heidelberg.

[69] Penrose, L. (1946). The elementary statistics of majority voting. J. R. Stat. Soc., 109:53-57.

[70] Rae, D. (1969). Decision-rules and individual values in constitutional choice. The American Political Science Review, 63(1):40-56.

[71] Riker, W. (1962). The theory of political coalitions, volume 578. Yale University Press New Haven.

[72] Schmeidler, D. (1969). The nucleolus of a characteristic function game. SIAM J. Appl. Math., 17:1163-1170.

[73] Shapiro, N. and Shapley, L. (1978). Values of large games, I: A limit theorem. Math. Oper. Res., 3(1):1-9.

[74] Shapley, L. (1953). A value for $n$-person games. Contrib. Theory of Games, Ann. Math. Stud.

[75] Shapley, L. and Shubik, M. (1954). A method for evaluating the distribution of power in a committee system. American Political Science Review, 48(03):787-792.

[76] Straffin, P. (1977). Homogeneity, independence, and power indices. Public Choice, 30(1):107-118.

[77] Taylor, A. and Zwicker, W. (1999). Simple games, desirability relations, trading, pseudoweightings. Princeton, New Jersey: Princeton University Press. 246 p.

[78] Tijs, S. (1981). Bounds for the core and the $\tau$-value. In O. Moeschlin et al., editor, Game Theory and Mathematical Economics, pages 123-132. North Holland Publishing Comp.

[79] Tsebelis, G. (2002). Veto players: How political institutions work. Princeton University Press.

[80] Weber, R. (1979). Subjectivity in the valuation of games. In Moeschlin, O. and Pallaschke, D., editors, Game Theory and Related Topics, pages 129-136. North Holland Publishing Comp.

[81] Widgren, M. (2001). On the probabilistic relationship between public good index and normalized Banzhaf index. In Holler, M. J. and Owen, G., editors, Power indices and coalition formation, pages 127-142. Springer.

\section{APPENDIX A. DETAILS FOR QUALITY FUNCTION RESULTS}

In this section we want to prove the missing details for the quality function results announced in Table 1. Since we have started with the proof for the Public Good Index, in Subsection 4.3, we want to continue with the Deegan-Packel index, which arises as the equal division of the PGI, see Definition 3.22

We can exploit this relation by setting $\widehat{C}^{\mathrm{DP}}=\sum_{i=k+1}^{n} C_{i}^{\mathrm{DP}}$ and denoting the number of minimal winning coalitions that contain at least one member of $(k, n]$ by $\widehat{M}^{\mathrm{PGI}}$. With this we have:

\section{Lemma A.1.}

$$
\widehat{M}^{\mathrm{PGI}} \leq \widehat{C}^{\mathrm{DP}} \cdot(k+1)
$$

Proof. Let $S$ be a minimal winning coalition that contains at least one player from $(k, n]$. We set $a:=|S \cap[1, k]|$ and $b:=|S \cap(k, n]|$, so that $a+b=|S|, 0 \leq a \leq k$, and $1 \leq b \leq n-k$. Thus, the stated inequality follows from $\sum_{i=k+1}^{n} C_{i}^{\mathrm{DP}}(v, S)=\frac{b}{a+b} \geq \frac{1}{k+1}$, where $v$ denotes the respective game.

Corollary A.2. For the Deegan-Packel index we can choose $f_{1}(k)=(k+1)^{2}$ and $f_{2}(k)=\frac{(k+1)^{2}(k+5)}{4}$.

Doing a tailored analysis similar to the one in the proof of Lemma 4.23 , allows us to provide tighter bounds:

Lemma A.3. For the Deegan-Packel index we can choose $f_{1}(k)=k+2$ and $f_{2}(k)=\frac{k^{2}+6 k+9}{4}$.

Proof. Let $v=(\mathcal{W},[n])$ be an arbitrary simple game. As defined before, $C_{i}^{\mathrm{DPI}}\left(v, 2^{[n]}\right)$ is the counting function of the Deegan-Packel index. For brevity, we just write $C_{i}$ and $\bar{C}$ for $C_{i}^{\mathrm{DPI}}$ and $\bar{C}^{\mathrm{DPI}}$. As further abbreviations we use $v^{\prime}=\left(\mathcal{W}^{\prime},[n]\right)$ for the $k$-rounding of $v$ and $\widehat{C}=\sum_{i=k+1}^{n} C_{i}$, i.e. the restriction of $\bar{C}$ to the players in $(k, n]$.

Now we want to study the changes in the $C_{i}$ by going from $v$ to $v^{\prime}$. At first we consider the cases where coalition $S$ is a MWC in $v$ but not in $v^{\prime}$.

(1) If $S \cap(k, n] \neq \emptyset$ then removing $S$ from $\mathcal{W}$ results in a decrease of at least $\frac{1}{|S|}$ for player $i$ and there is at least one player in $(k, n]$. Thus the negative change of the $C_{i}$ of that type is bounded by $\widehat{C}$.

(2) If $S \subseteq[1, k]$, then $S$ has to be winning in $v^{\prime}$ too. Since $S$ is not a MWC in $v^{\prime}$ (by assumption), there must be an index $j \in S$ such that $S-j$ is winning in $v^{\prime}$. Thus there must be a coalition $\emptyset \neq T \in 2^{(k, n]}$ such that $S-j \cup T$ is a MWC in $v$. So we can bound those cases by counting them at MWC $S-j \cup T$. The contribution of $S-j+T$ to $\widehat{C}$ is $\frac{t}{t+r} \geq \frac{1}{r+1}$, where $t=|T|$ and 
$r=|S-j|$. Given coalition $S-j+T$, there are $k-r \leq k-1$ possible choices for coalition $S$. In each choice the decrease of $C_{i}$ on $S$ is given by $\frac{1}{r+1}$ so that the total decrease of $C_{i}$ in those cases is bound from above by $(k-1) \widehat{C}$. For an upper bound of the decrease of all $C_{i}$ we have $k-r$ possibilities involving $r+1$ players each. Since $(k-r)(r+1) \leq\left\lfloor\frac{(k+1)^{2}}{4}\right\rfloor$, we have the upper bound $\left\lfloor\frac{(k+1)^{2}}{4}\right\rfloor \widehat{C}$.

Next, we consider the cases where coalition $S$ is not a MWC in $v$ but in $v^{\prime}$. Since the players in $(k, n]$ are null players we can deduce $S \in 2^{[1, k]}$.

(3) Assume that $S$ is losing in $v$ but winning in $v^{\prime}$. According to the rounding procedure there exists a coalition $\emptyset \neq T \in 2^{(k, n]}$ such that $S \cup T$ is a MWC in $v$. The contribution of $S \cup T$ to $\widehat{C}$ is $\frac{t}{t+r} \geq \frac{1}{r+1}$, where $t=|T|$ and $r=|S|$. Since $C_{i}(S)=\frac{1}{r}$ in $v$ and $r \geq 1$ we have that the total increase for $C_{i}$ is bounded by $2 \widehat{C}$. For the sum over the $C_{i}$ we note that the increase is at most $r \cdot \frac{1}{r}=1$ for a single coalition compared to $\frac{1}{r+1} \geq \frac{1}{k+1}$ so that the overall change of those cases is at most $(k+1) \widehat{C}$.

(4) If $S$ is winning in $v$, then there must be a player $j \in S$ such that $S-j$ is winning in $v$ but losing in $v^{\prime}$. We proceed similarly as in case (2) and deduce an upper bound of $k \widehat{C}$ for the change of $C_{i}$ and an upper bound of $\left\lfloor\frac{(k+1)^{2}}{4}\right\rfloor \widehat{C}$ for the sum of changes of the $C_{i}$.

Summarizing the four cases gives the mentioned two functions.

Lemma A.4. Let $v$ be a complete simple game with player set $N$ and $S \subseteq N$ a coalition. The number of direct left-shifts of $S$ is bounded by $|S|+1$ and $\left\lfloor\frac{n+1}{2}\right\rfloor$.

Proof. W.l.o.g. we assume $1 \succeq 2 \succeq \cdots \succeq n$. A direct left-shift of $S$ arises either by shifting a player of $S$ one place to the left or by adding the weakest player $n$. For the other bound we observe that player $i$ can only be shifted to position $i-1$ if $i-1 \notin S$ and $i \neq 1$. Thus only $\left\lfloor\frac{n}{2}\right\rfloor$ players can be shifted one position to the left, where equality is only possible if either $n$ is odd or $n$ is even and $n \in S$. Considering the possible addition of player $n$ given at most $\left\lfloor\frac{n+1}{2}\right\rfloor$ cases.

Lemma A.5. For the Shift index we can choose $f_{1}(k)=\frac{k+3}{2}$ and $f_{2}(k)=\frac{k^{2}+3 k+2}{2}$.

Proof. Let $v=(\mathcal{W},[n])$ be an arbitrary simple game. As defined before, $C_{i}^{\text {Shift }}\left(v, 2^{[n]}\right)$ counts the number of shift-minimal winning coalitions in $v$ containing player $i$ and we have $\bar{C}^{\text {Shift }}\left(v, 2^{[n]}\right)=$ $\sum_{i=1}^{n} C_{i}^{\text {Shift }}\left(v, 2^{[n]}\right)$. For brevity we just write $C_{i}$ and $\bar{C}$. As further abbreviations we use $v^{\prime}=$ $\left(\mathcal{W}^{\prime},[n]\right)$ for the $k$-rounding of $v, \widehat{C}=\sum_{i=k+1}^{n} C_{i}$, i.e. the restriction of $\bar{C}$ to the players in $(k, n]$, and by $\widehat{M}$ we denote the number of shift-minimal winning coalitions in $v$ that contain at least one player from $(k, n]$. With this we have $\widehat{M} \leq \widehat{C}$.

Now we want to study the changes in the $C_{i}$ by going from $v$ to $v^{\prime}$. At first we consider the cases where coalition $S$ is a SMWC (shift-minimal winning coalition) in $v$ but not in $v^{\prime}$.

(1) If $S \cap(k, n] \neq \emptyset$ then removing $S$ from $v$ results in a decrease of 1 for player $i$ and there is at least one player in $(k, n]$. Thus the negative change of the $C_{i}$ of that type is bounded by $\widehat{M} \leq \widehat{C}$.

(2) If $S \subseteq[1, k]$, then $S$ has to be winning in $v^{\prime}$ too. Since $S$ is not a SMWC in $v^{\prime}$ (by assumption), there must be a direct right-shift $S^{\prime}=S-j+h$ of $S$, where $j \in S, h \in[1, k] \backslash S$ or $h=\emptyset$, such that $S^{\prime}$ is winning in $v^{\prime}$. According to the rounding procedure, there has to be a subset $\emptyset \neq T \subseteq 2^{(k, n]}$ such that $S^{\prime} \cup T$ is a SMWC in $v$. Given $S^{\prime}$ there are at most $\frac{k+1}{2}$ choices for $S$, see Lemma A.4. Thus, we have that the negative change is bounded by $\frac{k+1}{2} \cdot \widehat{M} \leq \frac{k+1}{2} \cdot \widehat{C}$.

Next, we consider the cases where coalition $S$ is not a SMWC in $v$ but in $v^{\prime}$. Since the players in $(k, n]$ are null players, we can deduce $S \in 2^{[1, k]}$.

(3) Assume that $S$ is losing in $v$ but winning in $v^{\prime}$. According to the rounding procedure there exists coalition $\emptyset \neq T \in 2^{(k, n]}$ such that $S \cup T$ is a SMWC in $v$. Thus, the total increase for $C_{i}$ is bounded by $\widehat{M} \leq \widehat{C}$. 
(4) If $S$ is winning in $v$, then there must be a direct right-shift $S^{\prime}$ such that $S^{\prime}$ is winning in $v$ but losing in $v^{\prime}$. We proceed similarly as in case (2) and deduce an upper bound of $\frac{k+1}{2} \cdot \widehat{M}$ for the change of $C_{i}$.

Summarizing the four cases gives the mentioned two functions.

By proving the bound $\widehat{M}^{\text {Shift }} \leq \widehat{C}^{\text {SDP }} \cdot(k+1)$, similar to the proof of Lemma A.1, we can conclude:

$$
f_{1}(k)=\frac{k+3}{2} \text { and } f_{2}(k)=\frac{k^{2}+3 k+2}{2} .
$$

Corollary A.6. For the Shift Deegan-Packel index we can choose $f_{1}(k)=\frac{(k+1)(k+3)}{2}$ and $f_{2}(k)=$ $\frac{(k+1)^{2}(k+2)}{2}$.

But we can also perform a tailored analysis and improve the result a bit:

Lemma A.7. For the Shift Deegan-Packel index we can choose $f_{1}(k)=2 k+1$ and $f_{2}(k)=2 k^{2}+$ $k+1$.

Proof. Let $v=(\mathcal{W},[n])$ be an arbitrary simple game. As defined before, $C_{i}^{\mathrm{SDP}}\left(v, 2^{[n]}\right)$ is the counting function of the Shift Deegan-Packel index. For brevity we just write $C_{i}$ and $\bar{C}$ for $C_{i}^{\mathrm{SDP}}$ and $\bar{C}^{\mathrm{SDP}}$. As further abbreviations we use $v^{\prime}=\left(\mathcal{W}^{\prime},[n]\right)$ for the $k$-rounding of $v$ and $\widehat{C}=\sum_{i=k+1}^{n} C_{i}$, i.e. the restriction of $\bar{C}$ to the players in $(k, n]$.

Now we want to study the changes in the $C_{i}$ by going from $v$ to $v^{\prime}$. At first we consider the cases where coalition $S$ is a SMWC in $v$ but not in $v^{\prime}$.

(1) If $S \cap(k, n] \neq \emptyset$ then removing $S$ from $\mathcal{W}$ results in a decrease of $\frac{1}{|S|}$ for player $i$ and there is at least one player in $(k, n]$. Thus the negative change of the $C_{i}$ of that type is bounded by $\widehat{C}$.

(2) If $S \subseteq[1, k]$, then $S$ has to be winning in $v^{\prime}$ too. Since $S$ is not a SMWC in $v^{\prime}$ (by assumption), there must be a direct right-shift $S^{\prime}=S-j+h$ of $S$, where $j \in S, h \in[1, k] \backslash S$ or $h=\emptyset$, such that $S^{\prime}$ is winning in $v^{\prime}$. According to the rounding procedure there has to be a subset $\emptyset \neq T \subseteq 2^{(k, n]}$ such that $S^{\prime} \cup T$ is a SMWC in $v$. Let $u:=\left|S^{\prime}\right|$ and $v:=|T|$. Since $\frac{v}{u+v} \geq \frac{1}{u+1}$ the loss of the small ones is at least $\frac{1}{u+1}$. Since $u \leq|S| \leq u+1$ the decrease can be bounded by $2 k \widehat{C}$.

Next, we consider the cases where coalition $S$ is not a SMWC in $v$ but in $v^{\prime}$. Since the players in $(k, n]$ are null players we can deduce $S \in 2^{[1, k]}$.

(3) Assume that $S$ is losing in $v$ but winning in $v^{\prime}$. According to the rounding procedure, there exists coalition $\emptyset \neq T \in 2^{(k, n]}$ such that $S \cup T$ is a SMWC in $v$. Thus, the total increase for $C_{i}$ is bounded by $\widehat{C}$.

(4) If $S$ is winning in $v$, then there must be a direct right-shift $S^{\prime}$ such that $S^{\prime}$ is winning in $v G$ but losing in $v^{\prime}$. We proceed similarly as in case (2) and deduce an upper bound of $2 k \widehat{C}$ for the change of $C_{i}$.

Summarizing the four cases gives the mentioned two functions.

Lemma A.8. For the Tijs index (and $k$-up-rounding) we can choose $f_{1}(k)=0$ and $f_{2}(k)=1$.

Proof. Let $v$ denote the original game and $v^{\prime}$ the $k$-up-rounding of $v$ for an integer $0<k<n$. By $m_{1}$ we denote the number of vetoers in $[1, k]$ and by $m_{2}$ the number of vetoers in $(k, n]$ of $v . k$-uprounding maps winning coalitions to winning coalitions. A coalition $S$ is winning in $v^{\prime}$ iff $S \cap[1, k]$ is winning in $v^{\prime}$ and $S^{\prime} \subseteq[1, k]$ is winning in $v^{\prime}$ iff there exists a subset $T \subseteq(k, n]$ such that $S^{\prime} \cup T$ is winning in $v$. Thus a player $i \in[1, k]$ is a vetoer in $v$ if she is a vetoer in $v^{\prime}$ and no player $j \in(k, n]$ can be a vetoer in $v^{\prime}$. For all $1 \leq i \leq k$ we have $C_{i}^{\mathrm{Tijs}}\left(\Gamma(v), 2^{N}\right)=C_{i}^{\mathrm{Tijs}}\left(v, 2^{N}\right)$, so that we can choose $f_{1}(k)=0$, where $\Gamma$ denotes $k$-up-rounding. Since we have $\bar{C}^{\mathrm{Tijs}}\left(\Gamma(v), 2^{N}\right)=\bar{C}^{\mathrm{Tijs}}\left(v, 2^{N}\right)=m_{2}$, we can choose $f_{1}(k)=1$.

Lemma A.9. For ColPrev we can choose $f_{1}(k)=2$ and $f_{2}(k)=2 k+1$. 
Proof. Let us write $\eta_{i}\left(v, 2^{N}\right)=C_{i}^{\text {swing }}\left(v, 2^{N}\right)$ for the number of swings for player $i$ and $\bar{\eta}\left(v, 2^{N}\right)=$ $\bar{C}^{\text {swing }}\left(v, 2^{N}\right)$. By $v^{\prime}$ we denote $\Gamma(v)$, where $\Gamma$ is the $k$-rounding function. From Lemma 4.15 and Lemma4.12 we conclude

$$
\left|\eta_{i}\left(v^{\prime}, 2^{N}\right)-\eta_{i}\left(v, 2^{N}\right)\right| \leq \varepsilon \cdot \bar{\eta}\left(v, 2^{N}\right)
$$

for all $1 \leq i \leq k$. By $\mathcal{W}(v)$ and $\mathcal{W}\left(v^{\prime}\right)$ we denote the set of winning coalitions of $v$ and $v^{\prime}$, respectively. From the proof of Lemma 4.15 we obtain

$$
|| \mathcal{W}\left(v^{\prime}\right)|-| \mathcal{W}(v)|| \leq \varepsilon \cdot \bar{\eta}\left(v, 2^{N}\right) .
$$

With this we conclude

$$
\begin{aligned}
\left|\frac{\eta_{i}\left(v^{\prime}, 2^{N}\right)}{\left|\mathcal{W}\left(v^{\prime}\right)\right|}-\frac{\eta_{i}\left(v, 2^{N}\right)}{|\mathcal{W}(v)|}\right| & \leq\left|\frac{\eta_{i}\left(v^{\prime}, 2^{N}\right)}{|\mathcal{W}(v)|}-\frac{\eta_{i}\left(v, 2^{N}\right)}{|\mathcal{W}(v)|}\right|+\left|\frac{\eta_{i}\left(v^{\prime}, 2^{N}\right)}{\left|\mathcal{W}\left(v^{\prime}\right)\right|}-\frac{\eta_{i}\left(v^{\prime}, 2^{N}\right)}{|\mathcal{W}(v)|}\right| \\
& =\frac{\left|\eta_{i}\left(v^{\prime}, 2^{N}\right)-\eta_{i}\left(v, 2^{N}\right)\right|}{|\mathcal{W}(v)|}+\frac{\eta_{i}\left(v^{\prime}, 2^{N}\right)}{\left|\mathcal{W}\left(v^{\prime}\right)\right|} \cdot \frac{\left|\mathcal{W}\left(v^{\prime}\right)\right|-|\mathcal{W}(v)| \mid}{|\mathcal{W}(v)|} \\
& \leq \frac{\varepsilon \cdot \bar{\eta}\left(v, 2^{N}\right)}{|\mathcal{W}(v)|}+\frac{\eta_{i}\left(v^{\prime}, 2^{N}\right)}{\left|\mathcal{W}\left(v^{\prime}\right)\right|} \cdot \frac{\varepsilon \cdot \bar{\eta}\left(v, 2^{N}\right)}{|\mathcal{W}(v)|} \\
& \leq 2 \cdot \frac{\varepsilon \cdot \bar{\eta}\left(v, 2^{N}\right)}{|\mathcal{W}(v)|}
\end{aligned}
$$

where we have used the triangle inequality, Inequality (21), Inequality (22), and the fact that the number of swings for player $i$ cannot be larger then the number of winning coalitions. Thus we can choose $f_{1}(k)=2$. Due to Lemma 4.13 we can choose $f_{2}(k)=k \cdot f_{1}(k)+1=2 k+1$.

Lemma A.10. For ColIni we can choose $f_{1}(k)=2$ and $f_{2}(k)=2 k+1$.

Proof. As pointed out in e.g. [25] $\operatorname{ColIni}(v)$ equals $\operatorname{ColPrev}\left(v^{\star}\right)$, where $v^{\star}$ denotes the dual game of $v$, see e.g. [77] for a definition. Since the class of simple games is closed under taking the dual and $\widehat{\text { ColIni }}=\widehat{\text { ColPrev}}$, we can chose the same quality functions as in Lemma A.9.

While we have obtained a negative Alon-Edelman-type result for $p$-binomial semivalues, there are subclasses of semivalues where we can easily conclude a positive Alon-Edelman-type result from the proof of Lemma 4.15 .

Lemma A.11. Let $\Psi^{P}$ be a semivalue such that there exist real constants $c_{1}, c_{2} \in \mathbb{R}_{>0}$ such that $c_{1} \leq P_{i} \leq c_{2}$ for all $0 \leq i \leq n-1$. Then, we can choose $f_{1}(k)=\frac{c_{2}}{c_{1}}$ and $f_{2}(k)=\frac{c_{2}}{c_{1}} \cdot(k+1)$.

We remark that the above lemma is also valid for the more general probabilistic values if $c_{1} \leq$ $P_{S} \leq c_{2}$ holds for all $S \subseteq N$.

\section{APPENDIX B. DETAILS FOR A ClASS OF PARAMETERIZED WEIGHTED GAMES}

In this section we provide the delayed proofs from Subsection 4.4. Before going into the details for $p$-binomial semivalues and the (absolute) Johnston index, we provide a useful bound for binomial coefficients:

Lemma B.1. For integers $1 \leq m \leq n$ we have $\left(\begin{array}{c}n-1 \\ m-1\end{array}\right) \leq \frac{2^{n-1}}{\sqrt{n}}$.

Proof. Since the binomial coefficients attain their maximum at the center, it suffices to prove the proposed inequality for $m-1=\left\lfloor\frac{n-1}{2}\right\rfloor$. For $t \geq 1$ we have $\left(\begin{array}{c}2 t \\ t\end{array}\right) \leq \frac{2^{2 t}}{\sqrt{3 t+1}}$. From $\left(\begin{array}{c}2 t-1 \\ t-1\end{array}\right)+\left(\begin{array}{c}2 t-1 \\ t\end{array}\right)=$ $\left(\begin{array}{c}2 t \\ t\end{array}\right)$ and $\left(\begin{array}{c}2 t-1 \\ t-1\end{array}\right)=\left(\begin{array}{c}2 t-1 \\ t\end{array}\right)$, we conclude $\left(\begin{array}{c}2 t-1 \\ t-1\end{array}\right) \leq \frac{2^{2 t-1}}{\sqrt{3 t+1}}$ for all $t \geq 1$. Thus, we have $\left(\begin{array}{c}n-1 \\ m-1\end{array}\right) \leq \frac{2^{n-1}}{\sqrt{n}}$ for all $n \geq 1$. 
B.1. Technical details for $p$-binomial semivalues. We start our considerations with three technical estimates which will be used in the subsequent lemmas. The common theoretical basis of the first two estimates is Hoeffding's inequality, which provides an upper bound on the probability that the sum of random variables deviates from its expected value.

Lemma B.2. If $m=\left\lceil\frac{n+1}{2}\right\rceil$ and $p=\frac{1}{2}+\delta$, where $\frac{1}{2}>\delta>0$, then

$$
\sum_{j=m}^{n}\left(\begin{array}{l}
n \\
j
\end{array}\right) p^{j}(1-p)^{n-j} \geq 1-\exp \left(-2 n \delta^{2}\right)
$$

Proof. Since $\sum_{j=0}^{n}\left(\begin{array}{c}n \\ j\end{array}\right) p^{j}(1-p)^{n-j}=1$, it suffices to prove $\sum_{j=0}^{m-1}\left(\begin{array}{c}n \\ j\end{array}\right) p^{j}(1-p)^{n-j} \leq \exp \left(-2 n \delta^{2}\right)$. To this end, we apply Hoeffding's inequality for the special case of Bernoulli random variables and deduce

$$
\sum_{j=0}^{m-1}\left(\begin{array}{l}
n \\
j
\end{array}\right) p^{j}(1-p)^{n-j} \leq \exp \left(-2 \frac{(n p-(m-1))^{2}}{n}\right) \leq \exp \left(-2 \frac{(n \delta)^{2}}{n}\right)=\exp \left(-2 n \delta^{2}\right) .
$$

Lemma B.3. If $m=\left\lfloor\frac{n}{2}\right\rfloor$ and $p=\frac{1}{2}-\delta$, where $\frac{1}{2}>\delta>0$, then

$$
\sum_{j=0}^{m-1}\left(\begin{array}{l}
n \\
j
\end{array}\right) p^{j}(1-p)^{n-j} \geq 1-\exp \left(-2 n \delta^{2}+2 \delta\right) .
$$

Proof. Since $\sum_{j=0}^{m-1}\left(\begin{array}{l}n \\ j\end{array}\right) p^{j}(1-p)^{n-j}=\sum_{j=n-m+1}^{n}\left(\begin{array}{c}n \\ j\end{array}\right)(1-p)^{j} p^{n-j}$, we can similarly proceed as in the proof of Lemma B.2 and prove $\sum_{j=0}^{n-m}\left(\begin{array}{c}n \\ j\end{array}\right)(1-p)^{j} p^{n-j} \leq \exp \left(-2 n \delta^{2}+2 \delta\right)$. To this end, we apply Hoeffding's inequality for the special case of Bernoulli random variables and deduce

$$
\begin{aligned}
\sum_{j=0}^{n-m}\left(\begin{array}{c}
n \\
j
\end{array}\right) p^{j}(1-p)^{n-j} & \leq \exp \left(-2 \frac{(n(1-p)-(n-m))^{2}}{n}\right) \\
& \leq \exp \left(-2 \frac{\left(n \delta-\frac{1}{2}\right)^{2}}{n}\right) \leq \exp \left(-2 n \delta^{2}+2 \delta\right) .
\end{aligned}
$$

Lemma B.4. If $m \in\left\{\left\lceil\frac{n+1}{2}\right\rceil,\left\lfloor\frac{n}{2}\right\rfloor\right\}$ and $p=\frac{1}{2}+\delta$ or $p=\frac{1}{2}-\delta$, where $0 \leq \delta<\frac{1}{2}$, then

$$
p^{m-1}(1-p)^{n-m} \leq 4 \cdot \frac{\left(1-4 \delta^{2}\right)^{(n-3) / 2}}{2^{n-1}} .
$$

Proof. We have

$$
p^{m-1}(1-p)^{n-m}=\frac{(1 \pm 2 \delta)^{m-1} \cdot(1 \mp 2 \delta)^{n-m}}{2^{n-1}} \leq \frac{(1-2 \delta)^{(n-3) / 2} \cdot(1+2 \delta)^{(n+1) / 2}}{2^{n-1}},
$$

where the right hand side is bounded by $4 \cdot \frac{\left(1-4 \delta^{2}\right)^{(n-3) / 2}}{2^{n-1}}$ due to $\delta<\frac{1}{2}$.

Next we go on and determine formulas for the $p$-binomial semivalue $\Psi^{p}$ for the three games $v_{n, m}^{k, l}$, $v_{n, n+1}^{k, l}$, and $v_{n, 0}^{k, l}$ and the three types of players: 
Lemma B.5. For $p \in(0,1)$ and integers $l, k, m, n$ satisfying the restrictions from Definition 4.24 and $1 \leq m \leq n$, we have

$$
\begin{array}{rlrl}
\Psi_{i}^{p}\left(v_{n, m}^{k, l}\right)= & \left(\begin{array}{c}
k-1 \\
l-1
\end{array}\right) \cdot p^{l-1} \cdot(1-p)^{k-l} & & \\
& +1 \cdot p^{l}(1-p)^{k-l-1} \cdot \sum_{j=0}^{m-1}\left(\begin{array}{c}
n \\
j
\end{array}\right) \cdot p^{j}(1-p)^{n-j} & & \forall i \in[1, k-l] \\
\Psi_{i}^{p}\left(v_{n, m}^{k, l}\right)= & \left(\left(\begin{array}{c}
k-1 \\
l-1
\end{array}\right)-1\right) \cdot p^{l-1} \cdot(1-p)^{k-l} & & \\
& +1 \cdot p^{l-1}(1-p)^{k-l} \cdot \sum_{j=m}^{n}\left(\begin{array}{c}
n \\
j
\end{array}\right) \cdot p^{j}(1-p)^{n-j} & & \forall i \in(k-l, k] \\
\Psi_{i}^{p}\left(v_{n, m}^{k, l}\right)= & 1 \cdot p^{l}(1-p)^{k-l} \cdot\left(\begin{array}{c}
n-1 \\
m-1
\end{array}\right) \cdot p^{m-1}(1-p)^{n-m} & & \forall i \in(k, k+n] \\
\Psi_{i}^{p}\left(v_{n, n+1}^{k, l}\right)= & \left.\left(\begin{array}{c}
k-1 \\
l-1
\end{array}\right) \cdot p^{l-1} \cdot(1-p)^{k-l}+1 \cdot p^{l}(1-p)^{k-l-1}\right) & \forall i \in[1, k-l] \\
\Psi_{i}^{p}\left(v_{n, n+1}^{k, l}\right)= & \left(\left(\begin{array}{c}
k-1 \\
l-1
\end{array}\right)-1\right) \cdot p^{l-1} \cdot(1-p)^{k-l} & & \forall i \in(k-l, k] \\
\Psi_{i}^{p}\left(v_{n, n+1}^{k, l}\right)= & 0 & & \forall i \in(k, k+n] \\
\Psi_{i}^{p}\left(v_{n, 0}^{k, l}\right)= & \left(\begin{array}{c}
k-1 \\
l-1
\end{array}\right) \cdot p^{l-1} \cdot(1-p)^{k-l} & & \forall i \in[1, k-l] \\
\Psi_{i}^{p}\left(v_{n, 0}^{k, l}\right)= & \left(\begin{array}{c}
k-1 \\
l-1
\end{array}\right) \cdot p^{l-1} \cdot(1-p)^{k-l} & & \forall i \in(k-l, k] \\
\Psi_{i}^{p}\left(v_{n, 0}^{k, l}\right)= & 0 & & \forall i \in(k, k+n]
\end{array}
$$

Proof. We note that the $i$-swings of $v_{n, m}^{k, l}, v_{n, n+1}^{k, l}$, and $v_{n, 0}^{k, l}$ all are of one of the forms $U \subseteq[k]$ with $|U|=l-1$ and $T \cup V$ with $V \subseteq(k, k+n]$.

Now we are interested in the differences $\Psi^{p}\left(v_{n, m}^{k, l}\right)-\Psi^{p}\left(v_{n, n+1}^{k, l}\right)$ and $\Psi^{p}\left(v_{n, m}^{k, l}\right)-\Psi^{p}\left(v_{n, 0}^{k, l}\right)$. Inserting into the terms of Definition 4.27 yields:

Lemma B.6. For $p \in(0,1), P=\Psi^{p}$, and integers $l, k, m, n$ satisfying the restrictions from Definition 4.24 and $1 \leq m \leq n$, we have

$$
\begin{aligned}
\Delta_{1,2}^{1, P} & =p^{l}(1-p)^{k-l-1} \cdot \sum_{j=m}^{n}\left(\begin{array}{l}
n \\
j
\end{array}\right) p^{j}(1-p)^{n-j}, \\
\Delta_{1,2}^{2, P} & =p^{l-1}(1-p)^{k-l} \cdot \sum_{j=m}^{n}\left(\begin{array}{c}
n \\
j
\end{array}\right) p^{j}(1-p)^{n-j}, \\
\Delta_{1,2}^{3, P} & =1 \cdot p^{l}(1-p)^{k-l} \cdot\left(\begin{array}{c}
n-1 \\
m-1
\end{array}\right) \cdot p^{m-1}(1-p)^{n-m}, \\
\Delta_{1,3}^{1, P} & =p^{l}(1-p)^{k-l-1} \cdot \sum_{j=0}^{m-1}\left(\begin{array}{c}
n \\
j
\end{array}\right) p^{j}(1-p)^{n-j}, \\
\Delta_{1,3}^{2, P} & =p^{l-1}(1-p)^{k-l} \cdot \sum_{j=0}^{m-1}\left(\begin{array}{c}
n \\
j
\end{array}\right) p^{j}(1-p)^{n-j}, \\
\Delta_{1,3}^{3, P} & =1 \cdot p^{l}(1-p)^{k-l} \cdot\left(\begin{array}{c}
n-1 \\
m-1
\end{array}\right) \cdot p^{m-1}(1-p)^{n-m}, \text { and } \\
\xi^{P} & =n \Delta_{1,2}^{3, P}=n \Delta_{1,3}^{3, P}=n \cdot p^{l}(1-p)^{k-l} \cdot\left(\begin{array}{c}
n-1 \\
m-1
\end{array}\right) \cdot p^{m-1}(1-p)^{n-m} .
\end{aligned}
$$


Proof. (of Lemma4.28)

(1) We set $p=\frac{1}{2}+\delta$, where $\frac{1}{2}>\delta>0$, and conclude

$$
\frac{\Delta_{1,2}^{1, P}}{\xi^{P}}=\frac{\sum_{j=m}^{n}\left(\begin{array}{c}
n \\
j
\end{array}\right) p^{j}(1-p)^{n-j}}{n(1-p) \cdot\left(\begin{array}{c}
n-1 \\
m-1
\end{array}\right) \cdot p^{m-1}(1-p)^{n-m}} \geq \frac{1}{n(1-p)} \cdot \frac{1-\exp \left(-2 n \delta^{2}\right)}{\frac{2^{n-1}}{\sqrt{n}} \cdot 4 \frac{\left(1-4 \delta^{2}\right)^{(n-3) / 2}}{2^{n-1}}}
$$

using Lemma B.2, Lemma B.1, and Lemma B.4. The last expression can be rewritten to

$$
\frac{1}{4(1-p)} \cdot \frac{1-\exp \left(-2 n \delta^{2}\right)}{\sqrt{n}\left(1-4 \delta^{2}\right)^{(n-3) / 2}}
$$

which clearly tends to $\infty$ as $n$ increases. Since $\Delta_{1,2}^{2, P}=\Delta_{1,2}^{1, P} \cdot \frac{1-p}{p}$, we can also deduce the second statement.

(2) Similarly, we set $p=\frac{1}{2}-\delta$, where $\frac{1}{2}>\delta>0$, and conclude

$$
\frac{\Delta_{1,3}^{1, P}}{\xi^{P}}=\frac{\sum_{j=0}^{m-1}\left(\begin{array}{c}
n \\
j
\end{array}\right) p^{j}(1-p)^{n-j}}{n(1-p) \cdot\left(\begin{array}{c}
n-1 \\
m-1
\end{array}\right) \cdot p^{m-1}(1-p)^{n-m}} \geq \frac{1}{n(1-p)} \cdot \frac{1-\exp \left(-2 n \delta^{2}+2 \delta\right)}{\frac{2^{n-1}}{\sqrt{n}} \cdot 4 \frac{\left(1-4 \delta^{2}\right)^{(n-3) / 2}}{2^{n-1}}}
$$

using Lemma B.3. Lemma B.1, and Lemma B.4. The last expression can be rewritten to

$$
\frac{1}{4(1-p)} \cdot \frac{1-\exp \left(-2 n \delta^{2}+2 \delta\right)}{\sqrt{n}\left(1-4 \delta^{2}\right)^{(n-3) / 2}}
$$

which clearly tends to $\infty$ as $n$ increases. Since $\Delta_{1,3}^{2, P}=\Delta_{1,3}^{1, P} \cdot \frac{1-p}{p}$, we can also deduce the second statement.

(3) From Lemma B.1 and Lemma B.4 we conclude

$$
\xi^{P} \leq n \cdot\left(\begin{array}{c}
n-1 \\
m-1
\end{array}\right) \cdot p^{m-1}(1-p)^{n-m} \leq 4 \sqrt{n} \cdot\left(1-4 \delta^{2}\right)^{(n-3) / 2},
$$

where the right hand side clearly tends to zero as $n$ increases.

Proof. (of Lemma 4.29] For $i \in[1, k-l]$ we have

$$
\left|\Psi_{i}^{p}\left(v_{n, m}^{k, l}\right)-\Psi_{i}^{p}\left(v_{n, n+1}^{k, l}\right)\right|=\left|\Psi_{i}^{p}\left(v_{n, m}^{k, l}\right)-\Psi_{i}^{p}\left(v_{n, 0}^{k, l}\right)\right| \leq p^{l}(1-p)^{k-l-1} \leq p^{l-1}(1-p)^{k-l-1}
$$

and for $i \in(k-k, k]$ we have

$$
\left|\Psi_{i}^{p}\left(v_{n, m}^{k, l}\right)-\Psi_{i}^{p}\left(v_{n, n+1}^{k, l}\right)\right|=\left|\Psi_{i}^{p}\left(v_{n, m}^{k, l}\right)-\Psi_{i}^{p}\left(v_{n, 0}^{k, l}\right)\right| \leq p^{l-1}(1-p)^{k-l} \leq p^{l-1}(1-p)^{k-l-1} .
$$

Since we have defined $\frac{1}{n} \cdot \xi^{P}$ as

$$
\left|\Psi_{i}^{p}\left(v_{n, m}^{k, l}\right)-\Psi_{i}^{p}\left(v_{n, n+1}^{k, l}\right)\right|=\left|\Psi_{i}^{p}\left(v_{n, m}^{k, l}\right)-\Psi_{i}^{p}\left(v_{n, 0}^{k, l}\right)\right|,
$$

where $i \in(k, k+n]$, applying the triangle inequality for the absolute values gives the stated inequality. 


\section{B.2. Technical details for the (absolute) Johnston index.}

Lemma B.7. For $l=1$ and integers $k, m, n$ satisfying the restrictions from Definition 4.24 and $1 \leq m \leq n$, we have

$$
\begin{array}{rlrl}
\mathrm{JS}_{i}\left(v_{n, m}^{k, l}\right) & =\sum_{j=0}^{n}\left(\begin{array}{l}
n \\
j
\end{array}\right) \cdot 1+\sum_{j=0}^{m-1}\left(\begin{array}{l}
n \\
j
\end{array}\right) \cdot 1=2^{n}+\sum_{j=0}^{m-1}\left(\begin{array}{l}
n \\
j
\end{array}\right) & & \forall i \in[1, k) \\
\mathrm{JS}_{k}\left(v_{n, m}^{k, l}\right) & =\sum_{j=m+1}^{n}\left(\begin{array}{l}
n \\
j
\end{array}\right) \cdot 1+\left(\begin{array}{c}
n \\
m
\end{array}\right) \cdot \frac{1}{m+1} & \\
\mathrm{JS}_{i}\left(v_{n, m}^{k, l}\right) & =\left(\begin{array}{c}
n-1 \\
m-1
\end{array}\right) \cdot \frac{1}{m+1} & & \forall i \in(k, k+n] \\
\mathrm{JS}_{i}\left(v_{n, n+1}^{k, l}\right) & =\sum_{j=0}^{n}\left(\begin{array}{c}
n \\
j
\end{array}\right) \cdot 1=2^{n} & & \forall i \in[1, k] \\
\mathrm{JS}_{i}\left(v_{n, n+1}^{k, l}\right) & =0 & & \forall i \in(k, k+n] \\
\mathrm{JS}_{i}\left(v_{n, 0}^{k, l}\right) & =\sum_{j=0}^{n+1}\left(\begin{array}{c}
n \\
j
\end{array}\right) \cdot 1=2^{n+1} & & \forall i \in[1, k) \\
\operatorname{JS}_{i}\left(v_{n, 0}^{k, l}\right) & =0 & & \forall i \in[k, k+n] \\
\xi^{\mathrm{JS}} & =n \cdot\left(\begin{array}{c}
n-1 \\
m-1
\end{array}\right) \cdot \frac{1}{m+1} . &
\end{array}
$$

Proof. We note that all swing coalitions are of the form $\{i\} \cup T,\{i, k\} \cup T$, and $\{k\} \cup T$, where $1 \leq i \leq k-1$ and $\emptyset \subseteq T \subseteq(k, n+k]$.

Proof. (of Lemma 4.33) From Lemma B.1 and Lemma B.7 we conclude

$$
\begin{aligned}
& \mathrm{JS}_{i}\left(v_{n, m}^{k, l}\right)=3 \cdot 4^{\tilde{n}} \quad \forall i \in[1, k) \\
& \mathrm{JS}_{k}\left(v_{n, m}^{k, l}\right) \geq 4^{\tilde{n}}-\frac{\sqrt{2} \cdot 4^{\tilde{n}}}{\sqrt{\tilde{n}+1}} \\
& \mathrm{JS}_{k}\left(v_{n, m}^{k, l}\right) \leq 4^{\tilde{n}} \\
& \xi^{\mathrm{JS}} \leq \frac{\sqrt{2} \cdot 4^{\tilde{n}}}{\sqrt{\tilde{n}}} .
\end{aligned}
$$

For $\tilde{n} \geq 1$ we then have

$$
\begin{aligned}
\left\|\mathrm{JS}\left(v_{n, m}^{k, l}\right)-\mathrm{JS}\left(v_{n, n+1}^{k, l}\right)\right\|_{1} & \geq k \cdot 4^{\tilde{n}}+\xi^{\mathrm{JS}} \geq\left(k \cdot \frac{\sqrt{\tilde{n}}}{\sqrt{2}}+1\right) \xi^{\mathrm{JS}}, \\
\left\|\mathrm{JS}\left(v_{n, m}^{k, l}\right)-\mathrm{JS}\left(v_{n, 0}^{k, l}\right)\right\|_{1} & \geq k \cdot 4^{\tilde{n}}-\frac{\sqrt{2} \cdot 4^{\tilde{n}}}{\sqrt{\tilde{n}+1}}+\xi^{\mathrm{JS}} \geq(k-1) 4^{\tilde{n}}+\xi^{\mathrm{JS}} \\
& \geq\left((k-1) \cdot \frac{\sqrt{\tilde{n}}}{\sqrt{2}}+1\right) \xi^{\mathrm{JS}}, \text { and } \\
\lim _{\tilde{n} \rightarrow \infty} \frac{\xi^{\mathrm{JS}}}{\sum_{i=1}^{n+k} \mathrm{JS}_{i}\left(v_{n, m}^{k, l}\right)} & =0 .
\end{aligned}
$$


Proof. (of Lemma 4.35) Summing up the inequalities from Lemma 4.33 yields

$$
\begin{array}{r}
(3 k-2) \cdot 4^{\tilde{n}}-\frac{\sqrt{2} \cdot 4^{\tilde{n}}}{\sqrt{\tilde{n}}} \leq \sum_{i=1}^{k+n} \mathrm{JS}_{i}\left(v_{n, m}^{k, l}\right) \leq(3 k-2) \cdot 4^{\tilde{n}}+\frac{\sqrt{2} \cdot 4^{\tilde{n}}}{\sqrt{\tilde{n}}}, \\
\sum_{i=1}^{k+n} \operatorname{JS}_{i}\left(v_{n, n+1}^{k, l}\right)=(2 k) \cdot 4^{\tilde{n}}, \text { and } \\
\sum_{i=1}^{k+n} \operatorname{JS}_{i}\left(v_{n, 0}^{k, l}\right)=(4 k-4) \cdot 4^{\tilde{n}} .
\end{array}
$$

For $i \in[1, k)$ we have

$$
\left|\frac{\mathrm{JS}_{i}\left(v_{n, m}^{k, l}\right)}{\sum_{j=1}^{n+k} \mathrm{JS}_{j}\left(v_{n, m}^{k, l}\right)}-\frac{\mathrm{JS}_{i}\left(v_{n, n+1}^{k, l}\right)}{\sum_{j=1}^{n+k} \mathrm{JS}_{j}\left(v_{n, n+1}^{k, l}\right)}\right| \geq\left|\frac{3 \cdot 4^{\tilde{n}}}{(3 k-2) \cdot 4^{\tilde{n}}+\frac{\sqrt{2} \cdot 4^{\tilde{n}}}{\sqrt{\tilde{n}}}}-\frac{2 \cdot 4^{\tilde{n}}}{2 k \cdot 4^{\tilde{n}}}\right|,
$$

where the right hand side can be lower bounded by $\frac{1}{5 k(k-1)}$ for sufficiently large $\tilde{n}$, so that

$$
\sum_{i=1}^{n+k}\left|\frac{\mathrm{JS}_{i}\left(v_{n, m}^{k, l}\right.}{\sum_{j=1}^{n+k} \mathrm{JS}_{j}\left(v_{n, m}^{k, l}\right)}-\frac{\mathrm{JS}_{i}\left(v_{n, n+1}^{k, l}\right)}{\sum_{j=1}^{n+k} \mathrm{JS}_{j}\left(v_{n, n+1}^{k, l}\right)}\right| \geq \frac{1}{5 k} .
$$

Similarly we deduce

$$
\sum_{i=1}^{n+k}\left|\frac{\mathrm{JS}_{i}\left(v_{n, m}^{k, l}\right)}{\sum_{j=1}^{n+k} \mathrm{JS}_{j}\left(v_{n, m}^{k, l}\right)}-\frac{\mathrm{JS}_{i}\left(v_{n, 0}^{k, l}\right)}{\sum_{j=1}^{n+k} \mathrm{JS}_{j}\left(v_{n, 0}^{k, l}\right)}\right| \geq \frac{1}{5 k}
$$

Finally, we conclude

$$
\frac{\xi^{\mathrm{Js}}}{\sum_{j=1}^{n+k} \mathrm{JS}_{j}\left(v_{n, m}^{k, l}\right)} \leq \frac{\sqrt{2}}{(3 k-3) \sqrt{\tilde{n}}}
$$

for $\tilde{n} \geq 2$.

\section{APPENDIX C. ILP FORMULATIONS FOR THE COUNTING FUNCTIONS OF ALMOST ALL POWER INDICES FROM SECTION 3}

In Subsection 5.2 we have described the general idea of modeling power indices in the framework of integer linear programming. Our approach is based on the idea of a counting function. Since we can treat the normalized version of a power index, whenever we can treat the original version, see Subsection 5.3 for the algorithmic details, we restrict ourselve ${ }^{25}$ on presenting ILP formulations for the absolute versions of the power indices introduced in Section 3. In the following subsections we will state sufficient constraints for each power index separately. Those constraints for $y_{i, S}$ have to be required for all $i \in N$, which we will not repeat at each place.

C.1. SSI.

$$
\begin{array}{ll}
y_{i, S}=0 & \forall S \subseteq N \backslash\{i\}, \\
y_{i, S} \leq \frac{(|S|-1) !(n-|S|) !}{n !} \cdot x_{S} & \forall\{i\} \subseteq S \subseteq N, \\
y_{i, S} \leq \frac{(|S|-1) !(n-|S|) !}{n !} \cdot\left(1-x_{S \backslash\{i\}}\right) & \forall\{i\} \subseteq S \subseteq N \text { and } \\
y_{i, S} \geq \frac{(|S|-1) !(n-|S|) !}{n !} \cdot\left(x_{S}-x_{S \backslash\{i\}}\right) & \forall\{i\} \subseteq S \subseteq N .
\end{array}
$$

For subclasses of simple games this simplifies to

$$
\begin{array}{lrl}
y_{i, S}=0 & \forall S \subseteq N \backslash\{i\} \text { and } \\
y_{i, S}=\frac{(|S|-1) !(n-|S|) !}{n !} \cdot\left(x_{S}-x_{S \backslash\{i\}}\right) & \forall\{i\} \subseteq S \subseteq N .
\end{array}
$$

\footnotetext{
${ }^{25}$ Exact linearization techniques for fractional linear terms can e.g. be found in [55]. See also the approach described in Subsection C.7
} 
C.2. Tijs. As an incidence vector for minimal winning coalitions we introduce binary variables $z_{S}^{m} \in$ $\{0,1\}$ for all $S \subseteq N$. The constraints

$$
\begin{array}{ll}
z_{S}^{m} \leq x_{S} & \forall S \subseteq N, \\
z_{S}^{m} \leq 1-x_{S \backslash\{j\}} & \forall S \subseteq N, j \in S, \text { and } \\
z_{S}^{m} \geq x_{S}-\sum_{j \in S} x_{S \backslash\{j\}} & \forall S \subseteq N
\end{array}
$$

guarantee that $z_{S}^{m}=1$ if and only if $S$ is a minimal winning coalition. Next we introduce $m \in\{0,1\}$ with the interpretation that $m=1$ if and only if $\left|\mathcal{W}^{m}\right|=1$. This can be enforced by

$$
\begin{aligned}
m & \leq \sum_{S \subseteq N} z_{S}^{m} \\
m & \geq z_{S}^{m}-\sum_{T \subseteq N, T \neq S} z_{T}^{m} \quad \forall S \subseteq N, \text { and } \\
\sum_{S \subseteq N} z_{S}^{m} & \leq 1+(1-m) \cdot\left(2^{n}-1\right) .
\end{aligned}
$$

With this we can complete the ILP formulation by

for all $S \subseteq N$.

$$
\begin{aligned}
& y_{i, S} \leq z_{S}^{m} \\
& y_{i, S} \leq m, \text { and } \\
& y_{i, S} \geq z_{S}^{m}+m-1
\end{aligned}
$$

C.3. Semivalues $\Psi^{\mathrm{p}}$. As the Shapley-Shubik index is a special semivalue we might state an ILP formulation similar to those in Subsection C.1. In order to better highlight the underlying concept we introduce binary variables $z_{i, S}^{s} \in\{0,1\}$ for all $S \subseteq N$. The constraints

$$
\begin{array}{lll}
z_{i, S}^{s} \leq x_{S} & & \forall S \subseteq N, \\
z_{i, S}^{s} \leq 1-x_{S \backslash\{i\}} & & \forall S \subseteq N, \text { and } \\
z_{i, S}^{s} \geq x_{S}-x_{S \backslash\{i\}} & & \forall S \subseteq N
\end{array}
$$

guarantee that $z_{i, S}^{s}=1$ if and only if $i \in S$ and $S \backslash\{i\}$ is a swing coalition for $i$. For subclasses of simple games this simplifies to

$$
z_{i, S}^{s}=x_{S}-x_{S \backslash\{i\}} \quad \forall S \subseteq N .
$$

With this we can set $y_{i, S}=p_{|S|-1} \cdot z_{i, S}^{s}$.

C.4. Binomial semivalues $\Psi^{p}$. Using the notation from Subsection C.3 we set $y_{i, S}=p^{|S|-1}(1-$ $p)^{n-|S|} \cdot z_{i, S}^{s}$.

C.5. Bz. Using the notation from Subsection C.3 we set $y_{i, S}=1 / 2^{n-1} \cdot z_{i, S}^{s}$.

C.6. swing. Using the notation from Subsection C.3 we set $y_{i, S}=z_{i, S}^{s}$.

C.7. ColPrev. We use the notation from Subsection C.3. Things would be very easy if we could write $y_{i, S}=\frac{1}{|\mathcal{W}|} \cdot z_{i, S}^{s}$, but unfortunately even $\frac{1}{|\mathcal{W}|}$ is nonlinear, since $|\mathcal{W}|=\sum_{S \subseteq N} x_{S}$. Introducing the nonnegative real variables $a_{S} \in \mathbb{R}_{\geq 0}$ for all $S \subseteq N$, we can model the distribution of $\frac{1}{|\mathcal{W}|}$ to each winning coalition as follows:

$$
\begin{array}{rlrl}
a_{S} & \leq x_{S} & & \forall S \subseteq N, \\
a_{S}-a_{T} & \geq x_{S}+x_{T}-2 & \forall S, T \subseteq N, \text { and } \\
\sum_{S \subseteq N} a_{S} & =1 . & &
\end{array}
$$

By Inequality (42) we have $a_{S}=0$ for all losing coalitions and $a_{S} \leq 1$ for all winning coalitions. So especially we have $0 \leq a_{S} \leq 1$ for all $S \subseteq N$. Thus $a_{S}-a_{T} \geq-1$ for all $S, T \subseteq N$, so that Inequality (43) is only a restriction if both coalitions $S$ and $T$ are winning. In this case we have 
$a_{S}-a_{T} \geq 0$ and due to the symmetric formulation also $a_{T}-a_{S} \geq 0$, so that we finally have $a_{S}=a_{T}$ (Here we also have applied the Big-M method with $M=1$.). With Equation $\sqrt[44]{4}$ we obtain $a_{S}=\frac{1}{|\mathcal{W}|}$ for all winning coalitions $S$. It remains to assign the value $\frac{1}{|\mathcal{W}|}$ only to swing coalitions instead of winning coalitions:

$$
\begin{aligned}
& y_{i, S} \leq z_{i, S}^{s}, \\
& y_{i, S} \geq a_{S}-\left(1-z_{i, S}^{s}\right), \text { and } \\
& y_{i, S} \leq a_{S}+\left(1-x_{S}\right)
\end{aligned}
$$

for all $S \subseteq N$. If either $i \notin S$ or $S \backslash\{i\}$ is not a swing coalition for player $i$, then Inequality (45) ensures $y_{i, S}=0$ due to $y_{i, S} \geq 0$. Otherwise we have $z_{i, S}^{s}=x_{S}=1$, so that inequalities (46) and (47) are equivalent to $y_{i, S} \geq a_{S}$ and $y_{i, S} \leq a_{S}$, respectively. Thus we have $y_{i, S}=\frac{1}{|\mathcal{W}|}$ as requested. For either $z_{i, S}^{S}=0$ or $x_{S}=0$ inequalities (46) and (47) are satisfied automatically due to $0 \leq a_{S} \leq 1$.

C.8. ColIni. We similarly proceed as in Subsection C.7 and introduce the nonnegative real variables $a_{S} \in \mathbb{R}_{\geq 0}$, for all $S \subseteq N$. With this we can model the distribution of $\frac{1}{|\mathcal{L}|}$ to each losing coalition as follows:

$$
\begin{array}{rlrl}
a_{S} & \leq 1-x_{S} & \forall S \subseteq N, \\
a_{S}-a_{T} & \geq-x_{S}-x_{T} \quad \forall S, T \subseteq N, \text { and } \\
\sum_{S \subseteq N} a_{S} & =1 . &
\end{array}
$$

It remains to assign the value $\frac{1}{|\mathcal{L}|}$ only to swing coalitions instead of losing coalitions:

$$
\begin{array}{lll}
y_{i, S}=0 & \forall\{i\} \subseteq S \subseteq N, \\
y_{i, S} \leq 1-x_{S} & & \forall S \subseteq N \backslash\{i\}, \\
y_{i, S} \leq x_{S \cup\{i\}} & & \forall S \subseteq N \backslash\{i\}, \\
y_{i, S} \geq a_{S}-\left(x_{S}+1-x_{S \cup\{i\}}\right) & \forall S \subseteq N \backslash\{i\}, \text { and } \\
y_{i, S} \leq a_{S}+\left(x_{S}+1-x_{S \cup\{i\}}\right) & \forall S \subseteq N \backslash\{i\} .
\end{array}
$$

C.9. Rae.

$$
\begin{array}{ll}
y_{i, S}=\frac{1}{2^{|N|}} \cdot x_{S} & \forall\{i\} \subseteq S \subseteq N \text { and } \\
y_{i, S}=\frac{1}{2^{|N|}} \cdot\left(1-x_{S}\right) & \forall S \subseteq N \backslash\{i\} .
\end{array}
$$

C.10. KB. Using the notation from Subsection C.7 we can state

$$
\begin{array}{lll}
y_{i, S}=a_{S} & \forall\{i\} \subseteq S \subseteq N \text { and } \\
y_{i, S}=0 & \forall S \subseteq N \backslash\{i\} .
\end{array}
$$

C.11. PHI. Similarly as in Subsection C.7 we obtain:

$$
\begin{aligned}
y_{i, S} & =0 & & \forall S \subseteq N \backslash\{i\}, \\
y_{i, S} & \leq x_{S} & & \forall i\} \subseteq S \subseteq N, \\
y_{i, S} & \geq a_{S}-\left(1-x_{S}\right) & & \forall i\} \subseteq S \subseteq N, \\
y_{i, S} & \leq a_{S}+\left(1-x_{S}\right) & & \forall\{i\} \subseteq S \subseteq N, \\
a_{S} & \leq x_{S} & & \forall S \subseteq N, \\
a_{S}-a_{T} & \geq x_{S}+x_{T}-2 & & \forall S, T \subseteq N, \text { and } \\
\sum_{S \subseteq N}|S| \cdot a_{S} & =1 . & &
\end{aligned}
$$

C.12. Chow.

$$
\begin{array}{lll}
y_{i, S}=x_{S} & \forall\{i\} \subseteq S \subseteq N \text { and } \\
y_{i, S}=0 & \forall S \subseteq N \backslash\{i\} .
\end{array}
$$


C.13. JS. With the notation from Subsection $\overline{C .3}$ we can state:

$$
\begin{aligned}
y_{i, S} & \leq z_{i, S}^{s} & & \forall S \subseteq N, \\
y_{i, S}-y_{j, S} & \geq z_{i, S}^{s}+z_{j, S}^{s}-2 & & \forall i, j \in N, S \subseteq N, \\
\sum_{i=1}^{n} y_{i, S} & \leq 1 & & \forall S \subseteq N, \\
\sum_{i=1}^{n} y_{i, S} & \leq \sum_{i=1}^{n} z_{i, S}^{s} & & \forall S \subseteq N, \text { and } \\
\sum_{j=1}^{n} y_{j, S} & \geq z_{i, S}^{s} & & \forall S \subseteq N, i \in N .
\end{aligned}
$$

C.14. PGI.

$$
\begin{array}{lll}
y_{i, S}=0 & \forall S \subseteq N \backslash\{i\}, \\
y_{i, S} \leq x_{S} & \forall\{i\} \subseteq S \subseteq N, \\
y_{i, S} \leq 1-x_{S \backslash\{j\}} & \forall\{i\} \subseteq S \subseteq N, j \in S, \text { and } \\
y_{i, S} \geq x_{S}-\sum_{j \in S} x_{S \backslash\{j\}} & \forall\{i\} \subseteq S \subseteq N .
\end{array}
$$

C.15. DP.

$$
\begin{array}{ll}
y_{i, S}=0 & \forall S \subseteq N \backslash\{i\}, \\
y_{i, S} \leq \frac{1}{|S|} \cdot x_{S} & \forall\{i\} \subseteq S \subseteq N, \\
y_{i, S} \leq \frac{1}{|S|} \cdot\left(1-x_{S \backslash\{j\}}\right) & \forall\{i\} \subseteq S \subseteq N, j \in S, \text { and } \\
y_{i, S} \geq \frac{1}{|S|} \cdot\left(x_{S}-\sum_{j \in S} x_{S \backslash\{j\}}\right) & \forall\{i\} \subseteq S \subseteq N .
\end{array}
$$

C.16. Shift.

$$
\begin{array}{ll}
y_{i, S}=0 & \forall S \subseteq N \backslash\{i\}, \\
y_{i, S} \leq x_{S} & \forall\{i\} \subseteq S \subseteq N, \\
y_{i, S} \leq 1-x_{T} & \forall\{i\} \subseteq S \subseteq N, T \\
y_{i, S} \geq x_{S}-\sum_{T \text { direct right-shift of } S} x_{T} & \forall\{i\} \subseteq S \subseteq N .
\end{array}
$$

C.17. SDP.

$$
\begin{array}{rlrl}
y_{i, S}=0 & & \forall S \subseteq N \backslash\{i\} \\
y_{i, S} \leq \frac{1}{|S|} \cdot x_{S} & \forall\{i\} \subseteq S \subseteq N \\
y_{i, S} \leq \frac{1}{|S|} \cdot\left(1-x_{T}\right) & \forall\{i\} \subseteq S \subseteq N, T \text { direct right-shift of } S, \text { and (88) } \\
y_{i, S} \geq \frac{1}{|S|} \cdot\left(\begin{array}{c}
x_{S}-\sum_{T \text { direct right-shift of } S} x_{T} \\
\text { (87) }
\end{array}\right. & \forall\{i\} \subseteq S \subseteq N .
\end{array}
$$


APPENDIX D. COUNTING FUnCTIONS FOR ALMOST ALL POWER INDICES FROM SECTION 3

$$
\begin{aligned}
& C_{i}^{\mathrm{SSI}}(v, S)=\left\{\begin{aligned}
\frac{(|S|-1) !(n-|S|) !}{n !} & : \quad i \in S, v(S)=1, v(S-i)=0, \\
0 & : \text { otherwise, }
\end{aligned}\right. \\
& C_{i}^{\mathrm{Tijs}}(v, S)=\left\{\begin{array}{lll}
1 & : & S \in \mathcal{W}_{i}^{m},\left|\mathcal{W}^{m}\right|=1, \\
0 & : & \text { otherwise, }
\end{array}\right. \\
& C_{i}^{\Psi^{\mathbf{p}}}(v, S)=\left\{\begin{aligned}
p_{|S|-1} & : \quad i \in S, v(S)=1, v(S-i)=0, \\
0 & : \text { otherwise, }
\end{aligned}\right. \\
& C_{i}^{\Psi^{p}}(v, S)=\left\{\begin{aligned}
p^{|S|-1}(1-p)^{n-|S|} & : \quad i \in S, v(S)=1, v(S-i)=0, \\
0 & : \text { otherwise, }
\end{aligned}\right. \\
& C_{i}^{\mathrm{Bz}}(v, S)=\left\{\begin{aligned}
1 / 2^{n-1} & : \quad i \in S, v(S)=1, v(S-i)=0, \\
0 & : \text { otherwise }
\end{aligned}\right.
\end{aligned}
$$

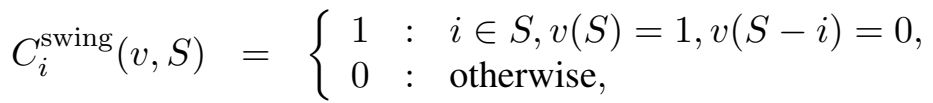

$$
\begin{aligned}
& C_{i}^{\text {ColPrev }}(v, S)=\left\{\begin{array}{rll}
\frac{1}{|\mathcal{W}|} & : & i \in S, v(S)=1, v(S-i)=0, \\
0 & : & \text { otherwise, }
\end{array}\right. \\
& C_{i}^{\text {ColIni }}(v, S)=\left\{\begin{array}{rll}
\frac{1}{|\mathcal{L}|} & : & i \notin S, v(S)=0, v(S+i)=1, \\
0 & : & \text { otherwise, }
\end{array}\right.
\end{aligned}
$$

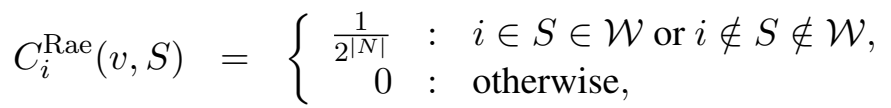

$$
\begin{aligned}
& C_{i}^{\mathrm{KB}}(v, S)=\left\{\begin{array}{rll}
\frac{1}{|\mathcal{W}|} & : & S \in \mathcal{W}_{i}, \\
0 & : & \text { otherwise },
\end{array}\right. \\
& C_{i}^{\mathrm{PHI}}(v, S)=\left\{\begin{array}{rll}
\frac{1}{\sum_{j=1}^{n}\left|\mathcal{W}_{j}\right|}: & S \in \mathcal{W}_{i}, \\
0 & : & \text { otherwise }
\end{array}\right. \\
& C_{i}^{\text {Chow }}(v, S)=\left\{\begin{array}{lll}
1 & : & S \in \mathcal{W}_{i} \\
0 & : & \text { otherwise }
\end{array}\right. \\
& C_{i}^{\mathrm{JS}}(v, S)=\left\{\begin{aligned}
\frac{1}{|\{j \in S: v(S-j)=0\}|} & : i \in S, v(S)=1, v(S-i)=0, \\
0 & : \text { otherwise, }
\end{aligned}\right. \\
& C_{i}^{\mathrm{PGI}}(v, S)=\left\{\begin{array}{lll}
1 & : & S \in \mathcal{W}_{i}^{m} \\
0 & : & \text { otherwise }
\end{array}\right. \\
& C_{i}^{\mathrm{DP}}(v, S)=\left\{\begin{array}{rll}
\frac{1}{|S|} & : & S \in \mathcal{W}_{i}^{m}, \\
0 & : & \text { otherwise }
\end{array}\right. \\
& C_{i}^{\text {Shift }}(v, S)=\left\{\begin{array}{lll}
1 & : & S \in \mathcal{W}_{i}^{s m}, \\
0 & : & \text { otherwise },
\end{array}\right. \\
& C_{i}^{\mathrm{SDP}}(v, S)=\left\{\begin{array}{rll}
\frac{1}{|S|} & : & S \in \mathcal{W}_{i}^{s m} \\
0 & : & \text { otherwise. }
\end{array}\right.
\end{aligned}
$$

\section{APPENDIX E. PROPERTIES OF POWER INDICES}

In Section 3 we have introduced several properties of power indices. Whether a certain power index satisfies such a property depends on the class of games where he is applied to. Here we want to restrict ourselves onto the class of simple games and summarize the results in Table 2 . Some of the stated entries can be e.g. found in [6], are folklore, or can be easily proven. Since all mentioned power indices $P$ are positive, we can apply Lemma 3.4 and deduce that the respective normalized power index $\widehat{P}$ is efficient. 


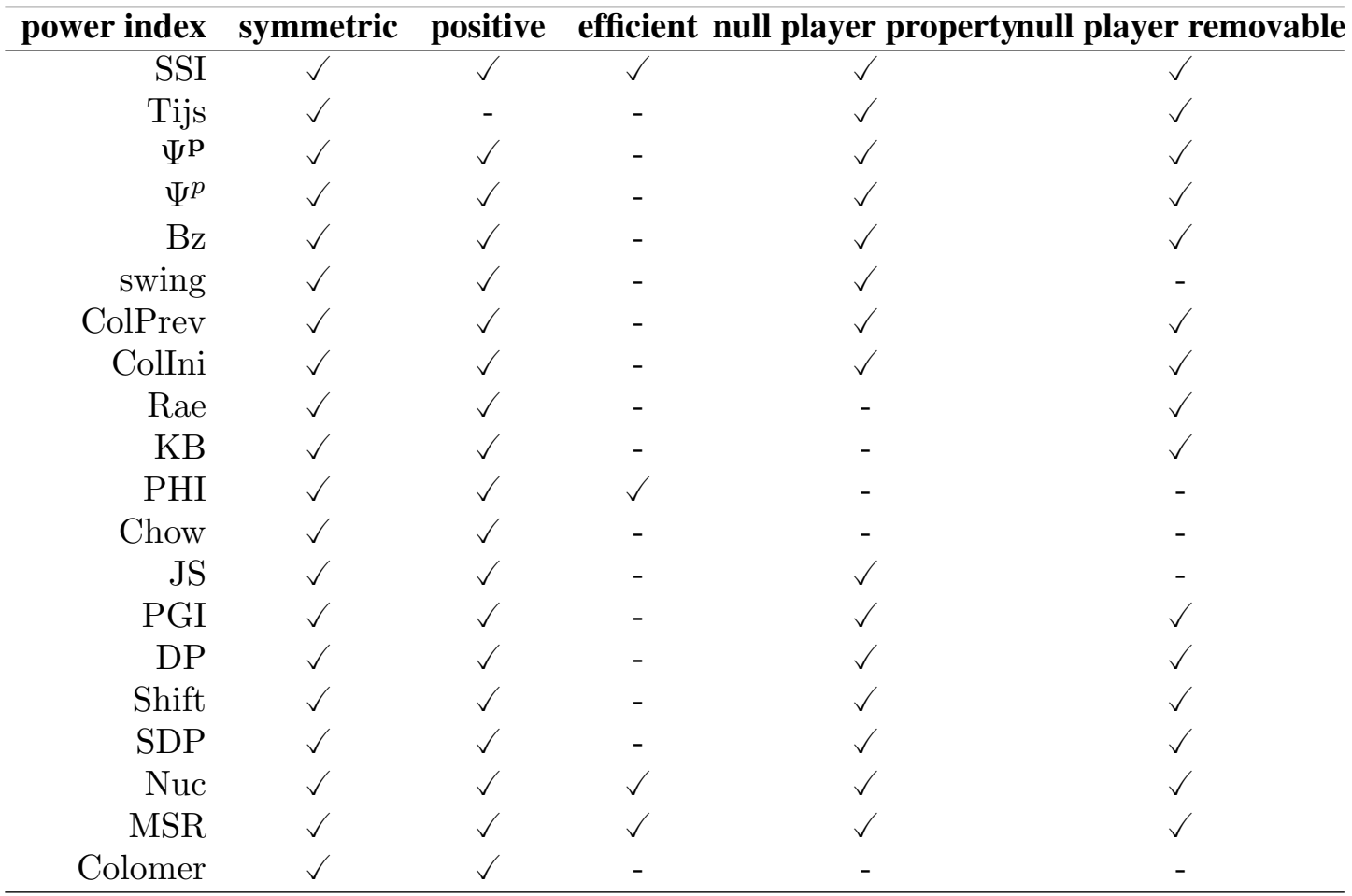

TABLE 2. Properties of power indices on simple games

\section{APPENDIX F. RECURSION FORMULAS FOR POWER INDICES}

Given the player set $N$, simple games are uniquely characterized by their set $\mathcal{W}$ of winning coalitions. With the inclusion operation for the sets of winning coalitions, simple games with player set $N$ become a poset and even a graded poset using the rank function $|\mathcal{W}|$, i.e. the number of winning coalitions. Starting from the simple game $v$, where all non-empty subsets are winning, we can reach each simple game by recursively turning a minimal winning coalition into a losing coalition. We can use this recursion for the computation of power indices of simple games. For each symmetric and efficient power index $P$ we have $P(v)=\frac{1}{n} \cdot(1, \ldots, 1)$. For other power indices the value $P(v)$ can usually be obtained easily. It remains to provide a recursive formula for a given power index for the case that one minimal winning coalition is turned into a losing coalition. For the swing count such a recursive formula is e.g. given in [29, Lemma 3.3.12]:

Lemma F.1. Let $v=(\mathcal{W}, N)$ be a simple game and $T \neq N$ be one of its minimal winning coalitions. Turning $T$ into a losing coalition gives a simple game $v^{\prime}$ with

$$
\eta_{i}\left(v^{\prime}\right)= \begin{cases}\eta_{i}(v)-1 & : \quad i \in T, \\ \eta_{i}(v)+1 & : \quad i \in N \backslash T\end{cases}
$$

for all $i \in N$.

Since $\mathrm{BZ}_{i}(v)=\frac{\eta_{i}(v)}{2^{n-1}}$ we obtain:

Lemma F.2. Let $v=(\mathcal{W}, N)$ be a simple game and $T \neq N$ be one of its minimal winning coalitions. Turning $T$ into a losing coalition gives a simple game $v^{\prime}$ with

$$
\mathrm{BZ}_{i}\left(v^{\prime}\right)=\left\{\begin{array}{l}
\mathrm{BZ}_{i}(v)-\frac{1}{2^{n-1}} \quad: \quad i \in T \\
\mathrm{BZ}_{i}(v)+\frac{1}{2^{n-1}} \quad: \quad i \in N \backslash T
\end{array}\right.
$$

for all $i \in N$.

From $\operatorname{Rae}_{i}(v)=\frac{1}{2}+\frac{1}{2} \cdot \mathrm{BZ}_{i}(v)$ we conclude:

Lemma F.3. Let $v=(\mathcal{W}, N)$ be a simple game and $T \neq N$ be one of its minimal winning coalitions. Turning $T$ into a losing coalition gives a simple game $v^{\prime}$ with

$$
\operatorname{Rae}_{i}\left(v^{\prime}\right)=\left\{\begin{array}{l}
\operatorname{Rae}_{i}(v)-\frac{1}{2^{n}} \quad: \quad i \in T \\
\operatorname{Rae}_{i}(v)+\frac{1}{2^{n}} \quad: \quad i \in N \backslash T
\end{array}\right.
$$


for all $i \in N$.

Also the Shapley-Shubik index counts swings in a weighted form, so that:

Lemma F.4. Let $v=(\mathcal{W}, N)$ be a simple game and $T \neq N$ be one of its minimal winning coalitions. Turning $T$ into a losing coalition gives a simple game $v^{\prime}$ with

$$
\operatorname{SSI}_{i}\left(v^{\prime}\right)=\left\{\begin{aligned}
\operatorname{SSI}_{i}(v)-\frac{(|T|-1) ! \cdot(|N|-|T|) !}{|N| !} & : \quad i \in T, \\
\operatorname{SSI}_{i}(v)+\frac{|T| ! \cdot(|N|-1-|T|) !}{|N| !} & : \quad i \in N \backslash T
\end{aligned}\right.
$$

for all $i \in N$.

More generally for $p$-binomial semivalues we have:

Lemma F.5. Let $p \in(0,1)$ and $v=(\mathcal{W}, N)$ be a simple game and $T \neq N$ be one of its minimal winning coalitions. Turning $T$ into a losing coalition gives a simple game $v^{\prime}$ with

$$
\Psi_{i}^{p}\left(v^{\prime}\right)= \begin{cases}\Psi_{i}^{p}(v)-p^{t-1}(1-p)^{n-t} & : \quad i \in T \\ \Psi_{i}^{p}(v)+p^{t}(1-p)^{n-t-1} & : \quad i \in N \backslash T\end{cases}
$$

for all $i \in N$, where $t=|T|$ and $n=|N|$.

We remark that the increments and the decrements differ by a factor of $\frac{p}{1-p}$ (or $\frac{1-p}{p}$ ). If only winning coalitions are counted, then only the value of the players in $T$ are changed:

Lemma F.6. Let $v=(\mathcal{W}, N)$ be a simple game and $T \neq N$ be one of its minimal winning coalitions. Turning $T$ into a losing coalition gives a simple game $v^{\prime}$ with

$$
\operatorname{Chow}_{i}\left(v^{\prime}\right)=\left\{\begin{aligned}
\operatorname{Chow}_{i}(v)-1 & : i \in T \\
\operatorname{Chow}_{i}(v) & : \quad i \in N \backslash T
\end{aligned}\right.
$$

for all $i \in N$.

For the König-Bräuninger index and the Coleman power of a member to prevent action we have to consider a reweighting of the values based on the change of the number of winning coalitions:

Lemma F.7. Let $v=(\mathcal{W}, N)$ be a simple game and $T \neq N$ be one of its minimal winning coalitions. Turning $T$ into a losing coalition gives a simple game $v^{\prime}$ with

$$
\mathrm{KB}_{i}\left(v^{\prime}\right)=\left\{\begin{aligned}
\frac{|\mathcal{W}|}{|\mathcal{W}|-1} \cdot \mathrm{KB}_{i}(v)-\frac{1}{|\mathcal{W}|-1} & : \quad i \in T, \\
\frac{|\mathcal{W}|}{|\mathcal{W}|-1} \cdot \mathrm{KB}_{i}(v) & : \quad i \in N \backslash T
\end{aligned}\right.
$$

and

$$
\operatorname{ColPrev}_{i}\left(v^{\prime}\right)= \begin{cases}\frac{|\mathcal{W}|}{|\mathcal{W}|-1} \cdot \operatorname{ColPrev}_{i}(v)-\frac{1}{|\mathcal{W}|-1} \quad: \quad i \in T, \\ \frac{|\mathcal{W}|}{|\mathcal{W}|-1} \cdot \operatorname{ColPrev}_{i}(v)+\frac{1}{|\mathcal{W}|-1} \quad: \quad i \in N \backslash T\end{cases}
$$

for all $i \in N$.

Lemma F.8. Let $v=(\mathcal{W}, N)$ be a simple game and $T \neq N$ be one of its minimal winning coalitions. Turning $T$ into a losing coalition gives a simple game $v^{\prime}$ with

$$
\mathrm{JS}_{i}\left(v^{\prime}\right)=\left\{\begin{aligned}
& \mathrm{JS}_{i}(v)-\frac{1}{|T|}: \quad i \in T, \\
& \mathrm{JS}_{i}(v)+\frac{1}{|T|+1} \quad: \quad i \in N \backslash T
\end{aligned}\right.
$$

for all $i \in N$.

For PGI, DP, Shift, and SDP it may be a non-trivial task to write down similar recursion formulas. 


\section{APPENDIX G. TeChNiCAL DETAILS FOR SHAPLEY-SHUBIK INDEX}

For the parametric class of weighted games $v_{n, m}^{k, l}$, defined in Subsection 4.4 and its possible $k$ roundings $v_{n, n+1}^{k, l}$ and $v_{n, 0}^{k, l}$, we want to deduce an explicit formula for the computation of the Shapley Shubik index for each player type.

For $v_{n, 0}^{k, l}$ the first $k$ players are symmetric and the other players are null players, so that we obtain

$$
\operatorname{SSI}\left(v_{n, 0}^{k, l}\right)=\left(\frac{1}{k}, \ldots, \frac{1}{k}, \frac{1}{k}, \ldots, \frac{1}{k}, 0, \ldots, 0\right)
$$

For $v_{n, n+1}^{k, l}$ and a player $i \in[1, k-l]$ the $i$-swings are given by the coalitions $U \cup V$, where $U \subseteq$ $[1, k]-i$ with $|U|=l-1, V \subseteq(k, k+n]$, and $T \cup V$, where $V \subseteq(k, k+n]$. Thus we obtain for the corresponding value of the SSI:

$$
\begin{aligned}
& \frac{1}{(n+k) !} \cdot\left(\sum _ { V \subseteq ( k , k + n ] } \left(\sum_{\substack{U \subseteq[1, k]-i \\
|U|=l-1}}|U \cup V| ! \cdot(n+k-|U \cup V|-1) !\right.\right. \\
& +|T \cup V| ! \cdot(n+k-|T \cup V|-1) !)) \\
= & \frac{1}{(n+k) !} \cdot\left(\sum_{j=0}^{n}\left(\begin{array}{c}
k-1 \\
l-1
\end{array}\right)\left(\begin{array}{c}
n \\
j
\end{array}\right)(l-1+j) !(n+k-l-j)+\sum_{j=0}^{n}\left(\begin{array}{c}
n \\
j
\end{array}\right)(l+j) !(n+k-l-j-1) !\right) \\
= & \frac{1}{k}+\frac{1}{k \cdot\left(\begin{array}{c}
k-1 \\
l
\end{array}\right)}
\end{aligned}
$$

Since the last $n$ players, in $(k, k+n]$, are null players, the central $l$ players are symmetric, and the Shapley-Shubik index is efficient, the Shapley vector for $v_{n, n+1}^{k, l}$ is given by $\operatorname{SSI}\left(v_{n, n+1}^{k, l}\right)=$

$$
\left(\frac{1}{k}+\frac{1}{k \cdot\left(\begin{array}{c}
k-1 \\
l
\end{array}\right)}, \ldots, \frac{1}{k}+\frac{1}{k \cdot\left(\begin{array}{c}
k-1 \\
l
\end{array}\right)}, \frac{1}{k}-\frac{k-l}{k l \cdot\left(\begin{array}{c}
k-1 \\
l
\end{array}\right)}, \ldots, \frac{1}{k}-\frac{k-l}{k l \cdot\left(\begin{array}{c}
k-1 \\
l
\end{array}\right)}, 0, \ldots, 0\right) .
$$

For $v_{m, n}^{k, l}$ the analysis is a bit more complicated. For $i \in(k, k+n)$ the $i$-swings are given by $T \cup V$ with $V \subseteq(k, k+n]-i$ and $|V|=m-1$, so that the Shapley value for those players is given by

$$
\begin{aligned}
& \frac{1}{(n+k) !} \cdot \sum_{\substack{V \subseteq(k, k+n]-i \\
|V|=m-1}}|T \cup V| ! \cdot(n-|T \cup V|-1) ! \\
= & \frac{1}{(n+k) !} \cdot\left(\begin{array}{c}
n \\
m-1
\end{array}\right)(l+m-1) !(n+k-m-l) !
\end{aligned}
$$

For $i \in[1, k-l]$ the $i$-swings are given by $U \cup V$ and $T \cup V^{\prime}$, where $V \subseteq(k, k+n], U \subseteq[1, k]-i$, $|U|=l-1$, and $V^{\prime} \subseteq(k, k+n],\left|V^{\prime}\right| \leq m-1$, so that the Shapley value for those players is given 
by

$$
\begin{aligned}
& \frac{1}{(n+k) !} \cdot\left(\sum_{\substack{U \subseteq[1, k]-i \\
|U|=l-l-1}} \sum_{V \subseteq(k, k+n]}|U \cup V| ! \cdot(n+k-|U \cup V|-1) !\right) \\
& +\frac{1}{(n+k) !} \cdot\left(\sum_{\substack{V^{\prime} \subseteq(k, k+n] \\
\left|V^{\prime}\right| \leq m-1}}\left|T \cup V^{\prime}\right| ! \cdot\left(n+k-\left|T \cup V^{\prime}\right|-1\right) !\right) \\
= & \frac{1}{(n+k) !} \cdot\left(\sum_{j=0}^{n}\left(\begin{array}{c}
k-1 \\
l-1
\end{array}\right)\left(\begin{array}{c}
n \\
j
\end{array}\right)(l-1+j) !(n+k-l-j) !\right. \\
& \left.+\sum_{j=0}^{m-1}\left(\begin{array}{l}
n \\
j
\end{array}\right)(l+j) !(n+k-l-j-1) !\right) \\
= & \frac{1}{k}+r_{k, l, n}^{m},
\end{aligned}
$$

where $r_{k, l, n}^{m}:=\frac{1}{(k+n) !} \cdot \sum_{j=0}^{m-1}\left(\begin{array}{c}n \\ j\end{array}\right)(l+j) !(n+k-l-j-1) !$ with $0 \leq r_{k, l, n}^{m} \leq \frac{1}{k\left(\begin{array}{c}k-1 \\ l\end{array}\right)}$.

We have done some numerical experiments with those formulas suggesting the existence of an Alon-Edelman type result for the Shapley-Shubik index.

Sascha Kurz, Department of Mathematics, Physics, and Computer Science, University of Bayreuth, 95440 Bayreuth, Germany. TEL.: +49-921-557353, FAX: +49-921-557352, SASCHA.KURZ@ UnI-BAYREUTH.DE 